\title{
Cosmetic surgery and the link volume of hyperbolic 3-manifolds
}

\author{
Yo’AV RIECK \\ YASUSHI YAMASHITA
}

We prove that for any $V>0$ there exists a hyperbolic manifold $M_{V}$ such that $\operatorname{Vol}\left(M_{V}\right)<2.03$ and $\operatorname{Link} \operatorname{Vol}\left(M_{V}\right)>V$. This was conjectured by the authors in [Algebr. Geom. Topol. 13 (2013) 927-958, Conjecture 1.3].

The proof requires study of cosmetic surgery on links (equivalently, fillings of manifolds with boundary tori). There is no bound on the number of components of the link (or boundary components). For statements, see the second part of the introduction. Here are two examples of the results we obtain:

(1) Let $K$ be a component of a link $L$ in $S^{3}$. Then "most" slopes on $K$ cannot be completed to a cosmetic surgery on $L$, unless $K$ becomes a component of a Hopf link.

(2) Let $X$ be a manifold and $\epsilon>0$. Then all but finitely many hyperbolic manifolds obtained by filling $X$ admit a geodesic shorter than $\epsilon$. (Note that it is not true that there are only finitely many fillings fulfilling this condition.)

57M12, 57M50

\section{Introduction}

In [19] we defined an invariant of closed orientable 3-manifolds that measures how efficiently a given manifold $M$ can be represented as a cover of $S^{3}$ where the branch set is a hyperbolic link (such a cover can be constructed using Hilden [8, Theorem 1] or Montesinos [15, Theorem 1]). We use the notation $M \stackrel{p}{\longrightarrow}\left(S^{3}, L\right)$ to denote a $p$-fold cover $M \rightarrow S^{3}$ branched over $L$. To the cover $M \stackrel{p}{\longrightarrow}\left(S^{3}, L\right)$ we associate the complexity $p \operatorname{Vol}\left(S^{3} \backslash L\right)$. The link volume of $M$ is defined to be the infimum of the complexities of all possible covers, that is,

$$
\operatorname{Link} \operatorname{Vol}(M)=\inf \left\{p \operatorname{Vol}\left(S^{3} \backslash L\right) \mid M \stackrel{p}{\longrightarrow}\left(S^{3}, L\right)\right\} .
$$

We observed that $\operatorname{Link} \operatorname{Vol}(M)>\operatorname{Vol}(M)$ for any hyperbolic manifold $M$, and conjectured that the link volume cannot be bounded in terms of the hyperbolic volume. In this paper we prove this conjecture. Our main result is the following (see the next two 
sections for definitions; by $M\left(\alpha_{i}\right)$ we mean the manifold obtained by filling $M$ along slope $\alpha_{i}$, and $d_{\text {Farey }}$ denotes the distance in the Farey graph):

Theorem 1.1 Let $M$ be a hyperbolic manifold with one cusp. Let $\left\{\alpha_{i}\right\}_{i \in I}$ be a set of slopes of $\partial M$. Then the following conditions are equivalent:

(1) There exists $L>0$ such that $\operatorname{Link} \operatorname{Vol}\left(M\left(\alpha_{i}\right)\right)<L$ for all $i \in I$.

(2) There exists $d>0$ such that $d_{\text {Farey }}\left(\alpha_{i}, \alpha_{i^{\prime}}\right) \leq d$ for all $i, i^{\prime} \in I$.

Remarks 1.2 (1) Corollary 1.8 of [19] shows that (2) implies (1); in fact, [19] allows for more than one boundary component and gives an explicit bound.

(2) Theorem 1.1 gives a partial answer to Conjecture 1.3 of [19].

Let $M$ denote the figure eight knot exterior; then $\operatorname{Vol}(M)=2.02988 \ldots$. By Cao and Meyerhoff [3, Theorem 1.1], $M$ has the smallest volume among all cusped hyperbolic 3-manifolds. Applying Theorem 1.1 to manifolds obtained by filling $M$, we get the following corollary:

Corollary 1.3 For every $V>0$, there exists a hyperbolic manifold $M_{V}$ such that

$$
\operatorname{Vol}\left(M_{V}\right)<2.02988 \ldots \text { and } \operatorname{LinkVol}\left(M_{V}\right)>V \text {. }
$$

This was conjectured by the author in their first study of the link volume (Conjecture 1.3 of [19]); this conjecture was the main motivation for the current paper, although as the reader will soon see, our investigation quickly led us to the study of cosmetic surgery on links.

This corollary can be interpreted (negatively) as saying that representing manifolds as branched covers of $S^{3}$ is inefficient. On the positive side, it shows that the link volume is a truly new invariant.

We refer the reader to [19] for basic facts and open questions about the link volume. We note that the link volume, which was defined using the hyperbolic volume, shares many of its basic properties.

Not much is known about the link volume of specific manifolds; Rieck and RemigioJuárez [18] calculated the link volume of certain prism manifolds (prism manifolds are small Seifert fibered spaces), but the link volume is not known for any hyperbolic manifold. A surprising feature of [18] is that the link volume is not finite-to-one. A few years ago Hitoshi Murakami asked if the link volume is multiplicative under unbranched covers. The motivation for the question is twofold: first, the hyperbolic volume has this property. Second, and more to the point, is the following construction: 
Let $\tilde{M} \rightarrow M$ be an unbranched cover between closed manifolds. Since $\tilde{M}$ and $M$ are closed, the degree of the cover is finite, and we will denote it by $p$. Then any (branched) cover $M \rightarrow S^{3}$ gives rise to a (branched) cover $\tilde{M} \rightarrow S^{3}$ via composition. The two covers have the same branch set and the degree is multiplicative; therefore the complexity of the covers multiplies. It follows that $\operatorname{Link} \operatorname{Vol}(\tilde{M}) \leq p \operatorname{Link} \operatorname{Vol}(M)$. The other direction is obviously tricky: given a (branched) cover $\tilde{M} \rightarrow S^{3}$, one only gets an induced cover $M \rightarrow S^{3}$ if the cover $\tilde{M} \rightarrow S^{3}$ is "equivariant" (we are using the term equivariant very loosely here, as the cover $\tilde{M} \rightarrow S^{3}$ need not be regular and so a group action may not exist). It is possible that Murakami's question has a positive answer for certain classes of manifolds but not in general: recently, Remigio-Juárez [17] constructed manifolds $M$ and $\tilde{M}$ together with covers $\tilde{M} \rightarrow M$ of arbitrarily high degree where $\operatorname{Link} \operatorname{Vol}(\tilde{M})=\operatorname{Link} \operatorname{Vol}(M)$.

In particular, the upper bound obtained in [19] is explicit, and linear in terms of distance in the Farey tessellation. It would be nice if a similar lower bound could be proved. The following question is obtained by simply reversing the inequality in the upper bound of [19, Corollary 1.8] (by $M\left(\alpha_{1}, \ldots, \alpha_{n}\right)$ we mean the manifold obtained by filling $M$ along slopes $\left.\alpha_{1}, \ldots, \alpha_{n}\right)$ :

Question 1.4 Let $M$ be a compact orientable connected 3-manifold with toral boundary. We will denote the components of $\partial M$ by $T_{1}, \ldots, T_{n}$. For each $i$, fix a slope $\beta_{i}$ of $T_{i}$.

Then do there exist $A, B>0$ such that, for any choice of slope $\alpha_{i}$ of $T_{i}$,

$$
\operatorname{LinkVol}\left(M\left(\alpha_{1}, \ldots, \alpha_{n}\right)\right)>-A+B\left(\sum_{i=1}^{n} d_{\text {Farey }}\left(\alpha_{i}, \beta_{i}\right)\right) ?
$$

The work in this paper is an application of the structure theorem of [19]. The structure theorem states that for any $V>0$, there is a finite set $\left\{\phi_{i}: X_{i} \rightarrow E_{i}\right\}_{i=1}^{n}$ of "parent systems", where $X_{i}$ and $E_{i}$ are hyperbolic manifolds and $\phi_{i}: X_{i} \rightarrow E_{i}$ is an unbranched cover, such that for any manifold $M$ with $\operatorname{Link} \operatorname{Vol}(M)<V$ there is some $i$ for which the following diagram commutes:

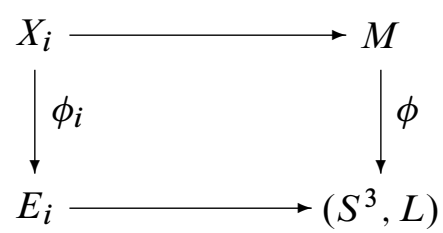

Here the horizontal arrows denote inclusions induced by fillings (that is, attaching solid tori) and $\phi: M \rightarrow S^{3}$ is a cover that realizes the link volume, that is, a cover for which 
$\operatorname{deg}(\phi) \operatorname{Vol}\left(S^{3} \backslash L\right)=\operatorname{LinkVol}(M)$. The reader may observe the similarity to the celebrated result of Jørgensen and Thurston [24] that states that for every $V>0$ there are finitely many "parent manifolds" $\left\{E_{i}\right\}_{i=1}^{n}$ such that every hyperbolic manifold of volume less than $V$ can be obtained by filling $E_{i}$ for some $i$. (For a detailed exposition see, for example, Kobayashi and Rieck [14].) This is no coincidence; the structure theorem is a consequence of Jørgensen-Thurston and the manifolds $E_{i}$ appearing in it are the parent manifolds of Jørgensen and Thurston.

In order to obtain Theorem 1.1 from the structure theorem above, we are forced to study several questions about fillings. Specifically, we study several questions about cosmetic surgery, that is, surgery on a link in a manifold $M$ that results in a manifold diffeomorphic to $M$. Before describing our methods and the results obtained, we introduce the basic setup; detailed description is given in Section 2A.

After two preliminary sections (2 and 3), in Sections 4 and 5 we construct our main tool, a rooted tree denoted $T(X)$ which we associate with a manifold $X$. The vertices of $T(X)$ are labeled by manifolds; $X$ itself corresponds to the root of $T(X)$. If $X$ is hyperbolic, its immediate descendants are certain nonhyperbolic manifolds obtained by filling $X$; if $X$ is not prime, its immediate descendants are the factors of its prime decomposition; if $X$ is JSJ (that is, if $X$ is prime and the collection of tori in the JSJ decomposition of $X$ is not empty), its immediate descendants are the components of its torus decomposition. If $X$ is Seifert fibered or sol it has no descendants. We prove (Proposition 5.1) that $T(X)$ is finite. All the results described below are proved by induction on $|T(X)|$, the number of vertices in $T(X)$. The various applications of $T(X)$ are somewhat independent, and we made an effort to make the subsequent sections (especially Sections 6-10 and 12) independently readable. Throughout this paper a set of slopes of a torus is called bounded if it is bounded in the Farey graph.

In Section 6 we study cosmetic surgery on a link $L \subset T^{2} \times[0,1]$. Let $B$ be a bounded set of slopes of $T^{2} \times\{1\}$. By a multislope $\alpha$ of $L$ we mean a vector whose components are slopes on the components of $L$ or $\infty$ (see Section 2A for a precise definition). We will denote the manifold obtained by surgery on $L$ with multislope $\alpha$ by $L(\alpha)$. Let $\mathcal{A}$ be the multislopes of $L$ that yield cosmetic surgery, that is, $\mathcal{A}=\left\{\alpha \mid L(\alpha) \cong T^{2} \times[0,1]\right\}$. Given $\alpha \in \mathcal{A}$, we may use the product structure of $L(\alpha)$ to project the set $B$ and obtain a set of slopes of $T^{2} \times\{0\}$. Since this set depends on $\alpha$ we will denote it by $B_{\alpha}$. We prove the $T^{2} \times[0,1]$ cosmetic surgery theorem (Theorem 6.1), which says that

$$
\bigcup_{\alpha \in \mathcal{A}} B_{\alpha}
$$

is a bounded set of slopes of $T^{2} \times[0,1]$. 
In Section 7 we study cosmetic surgery on a link $L \subset D^{2} \times S^{1}$. We prove the solid torus cosmetic surgery theorem (Theorem 7.1), which says that the set of slopes of $\partial D^{2} \times S^{1}$ that bound a disk after cosmetic surgery on $L$ is bounded (unless some component of $L$ is a core of the solid torus after surgery, in which case the claim is obviously false). For use in later sections we also prove Proposition 7.2, which gives certain constraints on multislopes of $L$ that yield a cosmetic surgery.

Sections 8 and 9 are devoted to cosmetic surgery on hyperbolic manifolds. Let $M$ be a hyperbolic manifold, $L \subset M$ a link, $T$ a component of $\partial M, B$ a bounded set of slopes of $T$, and $\mathcal{X}=\left\{\left(\alpha, f_{\alpha}\right)\right\}$ such that, for every $\left(\alpha, f_{\alpha}\right), \alpha$ is a multislope of $L$ and $f_{\alpha}$ is a diffeomorphism $f_{\alpha}: L(\alpha) \rightarrow M$ that maps $T$ to itself. Then for every $\left(\alpha, f_{\alpha}\right) \in \mathcal{X}$ the image of $B$ under $f_{\alpha}$ is a set of slopes of $T$ that we will denote by $B_{\alpha, f_{\alpha}}$. In Section 8 we prove Theorem 8.1 , which says that

$$
\bigcup_{\left(\alpha, f_{\alpha}\right) \in \mathcal{X}} B_{\alpha, f_{\alpha}}
$$

is a bounded set of slopes of $T$.

In Section 9 we consider multislopes $\alpha$ of a manifold $X$ that yield a hyperbolic manifold $X(\alpha)$ such that every geodesic in $X(\alpha)$ is longer than $\epsilon$ (for some fixed $\epsilon>0$; here $\alpha$ is a multislope on $\partial X$ and $X(\alpha)$ represents filling rather than surgery). We prove two things: First, there are only finitely many such manifolds $X(\alpha)$ (although there certainly may be infinitely many such multislopes $\alpha$ ). Second, we prove that all but finitely many of these multislopes factor through a nonhyperbolic filling. In other words, there is a subset of the boundary components such that the manifold obtained by filling only these components is not hyperbolic (we call this a nonhyperbolic partial filling; this and other useful terminology is introduced in Section 2A). Moreover, one of the nonhyperbolic partial fillings corresponds to an edge out of the root of $T(X)$; this is the key that allows us to use induction.

In Section 10 we prove the $S^{3}$ cosmetic surgery theorem (Theorem 10.1): let $L \subset S^{3}$ be a link and $K$ a component of $L$. Let $\mathcal{A}=\left\{\alpha \mid L(\alpha)=S^{3}\right\}$ and $\mathcal{A}^{\prime} \subset \mathcal{A}$ be the multislopes for which the core of the solid torus attached to $\partial N(K)$ does not form a Hopf link with the core of any other attached solid torus. Given a multislope $\alpha$, we denote its value on $K$ by $\left.\alpha\right|_{K}$. We prove that

$$
\left\{\left.\alpha\right|_{K} \mid \alpha \in \mathcal{A}^{\prime}\right\}
$$

is bounded. In Example 10.3 we show that the bound cannot be given explicitly in terms of the number of components of $L$ : for any $d>0$ we construct a 2-component link $L$ with diameter at least $d$. Since Theorem 10.1 is used in subsequent sections, 
none of the sets shown to be bounded there can be given an explicit bound; moreover, similar constructions can be made for previous sections.

Finally, in Sections 11-14 we apply these results to prove Theorem 1.1. In particular, in Section 12 we prove Theorem 12.1, which is of independent interest. In it we consider manifolds $X$ and $M$, where $M$ is a one-cusped hyperbolic manifold. We consider the set of multislopes $\mathcal{A}$ of $\partial X$ such that any $\alpha \in \mathcal{A}$ fulfills the following condition: the manifold obtained by filling all but one boundary component of $X$ is not $M$. We describe this by saying that $\alpha$ does not admit a partial filling $\alpha^{\prime}$ for which $X\left(\alpha^{\prime}\right) \cong M$. In Theorem 12.1 we show that the set of slopes

$$
\{\beta \mid M(\beta) \cong X(\alpha) \text { for some } \alpha \in \mathcal{A}\}
$$

is bounded.

Acknowledgements We thank Matt Day, Chaim Goodman-Strauss, Mike Hilden, Kazuhiro Ichihara, Tsuyoshi Kobayashi, Marc Lackenby, Kimihiko Motegi, John Retcliffe and Masakazu Teragaito for helpful conversations and correspondence. We thank the anonymous referee for a very careful reading of the paper and many helpful comments. A very special thank-you to Jair Remigio-Juárez and Michael Yoshizawa, whose patient reading of this paper helped us greatly. Yo'av Rieck: this research took place during three visits to Nara Women's University. I would like to thank the Math Department and the Department of Information and Computer Science for their hospitality during my long stays.

This work was partially supported Simons Foundation grant 283495 to Yo'av Rieck.

\section{Background}

Throughout this paper, by manifold we mean a compact orientable connected 3dimensional smooth manifold. We only consider manifolds with toral boundary, that is, manifolds whose boundary consists of a (possibly empty) collection of tori. A manifold is called hyperbolic if its interior admits a complete finite-volume Riemannian metric locally isometric to hyperbolic space $\mathbb{H}^{3}$; we sometimes refer to the boundary components of a hyperbolic manifold as cusps. We denote the closed normal neighborhood, closure, and interior by $N(\cdot), \mathrm{cl}$, and int, respectively. The geometric intersection number between curves on a torus is denoted $\Delta(\cdot, \cdot)$. Given a knot or a $\operatorname{link} L \subset M$, we call $M \backslash$ int $N(L)$ the exterior of $L$ and denote it by $E(L)$. We always assume transversality. 


\section{A Notation}

The following notation will be used extensively throughout the paper. Let $X$ be a manifold, fix $n$ components of $\partial X$, denoted by $T_{1}, \ldots, T_{n}$, and denote their union by $\mathcal{T}=\bigcup_{i=1}^{n} T_{i}$ (note that $\mathcal{T} \subset \partial X$, but possibly $\mathcal{T} \neq \partial X$ ).

(1) By a multislope of $\mathcal{T}$, say $\alpha$, we mean a vector $\alpha=\left(\alpha_{1}, \ldots, \alpha_{n}\right)$ such that, for each $i$, either $\alpha_{i}$ is a slope on $T_{i}$ (that is, the homology class of a connected simple closed curve on $T_{i}$ ) or $\alpha_{i}=\infty$. By a multislope of a link $L$ we mean a multislope of $\partial N(L) \subset \partial E(L)$.

(2) By filling $X$ along $\alpha$ we mean the manifold $X(\alpha)$ that is obtained by the following operation:

(a) To components $T_{i} \subset \mathcal{T}$ for which $\alpha_{i} \neq \infty$ we attach a solid torus $V_{i}$ so that the meridian of $V_{i}$ is identified with a connected simple closed curve representing $\alpha_{i}$.

(b) Nothing is done to components $T_{i} \subset \mathcal{T}$ for which $\alpha_{i}=\infty$ and components of $\partial X \backslash \mathcal{T}$.

(3) If $\alpha=\left(\alpha_{1}, \ldots, \alpha_{n}\right)$ and $\alpha^{\prime}=\left(\alpha_{1}^{\prime}, \ldots, \alpha_{n}^{\prime}\right)$ are multislopes, we say that $\alpha^{\prime}$ is a partial filling of $\alpha$, and write $\alpha^{\prime} \subset_{\mathrm{pf}} \alpha$, if $\alpha_{i}^{\prime} \in\left\{\alpha_{i}, \infty\right\}$ for each $i$. If $\alpha^{\prime} \subset \mathrm{pf}$ and $\alpha^{\prime} \neq \alpha$ we say that $\alpha^{\prime}$ is a strict partial filling of $\alpha$. We will also use the notation $\left(\alpha_{1}, \ldots, \widehat{\alpha}_{i}, \ldots, \alpha_{n}\right)$ for the multislope obtained from $\left(\alpha_{1}, \ldots, \alpha_{i}, \ldots, \alpha_{n}\right)$ by replacing $\alpha_{i}$ with $\infty$ (intuitively, tossing $\alpha_{i}$ out).

(4) Assume that $X$ is hyperbolic. A multislope $\alpha$ is called hyperbolic if $X(\alpha)$ is hyperbolic; $\alpha$ is called totally hyperbolic if every partial filling of $\alpha$ is hyperbolic.

(5) Assume that $X$ is hyperbolic. A multislope $\alpha$ is called nonhyperbolic if $X(\alpha)$ is not hyperbolic. If $\alpha$ is nonhyperbolic, and every strict partial filling of $\alpha$ is hyperbolic, then $\alpha$ is called minimally nonhyperbolic. Minimally nonhyperbolic fillings play a key role in our work and are studied extensively in Section 4.

(6) Let $\mathcal{T}, \mathcal{T}^{\prime}$ be unions of components of $\partial X$ and $\alpha$ a multislope of $\mathcal{T}$. Then $\alpha$ defines a multislope on $\mathcal{T}^{\prime}$ by ignoring the components of $\alpha$ that correspond to components of $\mathcal{T} \backslash \mathcal{T}^{\prime}$ and assigning the value $\infty$ to every component of $\mathcal{T}^{\prime} \backslash \mathcal{T}$. This multislope is called the restriction of $\alpha$ to $\mathcal{T}^{\prime}$ and we will denote it by $\left.\alpha\right|_{\mathcal{T}^{\prime}}$. In particular, we will denote the value of $\alpha$ on $T_{i}$ by $\left.\alpha\right|_{T_{i}} ;\left.\alpha\right|_{\mathcal{T}^{\prime}}$ is also called the multislope induced by $\alpha$ on $\mathcal{T}^{\prime}$.

(7) Induced multislopes also appear in a more general setting. Let $F$ be a collection of tori in $\operatorname{int}(X)$. Suppose that $X$ cut open along $F$ consists of $N_{1}$ and $N_{2}$, that is, $X=N_{1} \cup_{F} N_{2}$. Here we are not assuming that $N_{1}$ or $N_{2}$ is connected. Let $\alpha$ be a multislope of components of $\partial X$ (denoted $\mathcal{T}$ ) such that, for each 
component $Y$ of $N_{2}$, we have $Y\left(\left.\alpha\right|_{\partial Y}\right) \cong D^{2} \times S^{1}$. In other words, after filling, $N_{2}\left(\left.\alpha\right|_{\partial N_{2}}\right)$ consists of solid tori. We will denote $\left(\mathcal{T} \cap \partial N_{1}\right) \cup F$ by $\mathcal{T}_{1} \subset \partial N_{1}$. Then the multislope of $\mathcal{T}_{1}$ induced by $\alpha$ is the multislope defined by $\left.\alpha\right|_{\mathcal{T} \cap \partial N_{1}}$ (on the components of $\mathcal{T} \cap \partial N_{1}$ ) and the homology classes of the meridians of $N_{2}\left(\left.\alpha\right|_{\partial N_{2}}\right)$ (on the components of $F$ ).

(8) Recall that if $L \subset X$ is a link then a multislope of $L$ is a multislope of $\partial N(L) \subset$ $\partial E(L)$. By surgery on $L$ along the multislope $\alpha$, which we will denote by $L(\alpha)$, we mean $E(L)(\alpha)$.

\section{B JSJ-manifolds}

In this subsection we summarize the information we need about manifolds with nontrivial JSJ decomposition. JSJ decompositions were studied by Jaco and Shalen [10] and, independently, by Johannson [11]. We assume familiarity with this subject; further details can be found in [9]. We summarize what we need in the definition below; note that since we restrict our attention to manifolds with boundary tori we may assume that the JSJ decomposition is along tori (and no annuli).

Definition 2.1 (JSJ-manifold) Let $X$ be a compact 3-manifold such that $\partial X$ consists of a (possibly empty) collection of tori. We say that $X$ is a JSJ-manifold if $X$ is irreducible and the JSJ-decomposition of $X$ consists of a nonempty collection of tori; in that case we also say that $X$ has a nontrivial JSJ decomposition. Let $\mathcal{T}$ denote the tori in the torus decomposition of a JSJ manifold $X$. The manifolds obtained by cutting $X$ along $\mathcal{T}$ are called the components of the torus decomposition of $X$. The graph dual to the JSJ decomposition of $X$, or the graph dual to $\mathcal{T}$, is the simplicial graph with one vertex for every component of the torus decomposition of $X$ and an edge for every torus in $\mathcal{T}$; the edge corresponding to $T \in \mathcal{T}$ connects the (not necessarily distinct) vertices that correspond to the components of the torus decomposition of $X$ that have $T$ in their boundary.

\section{C Topological preliminaries}

We will need the following simple lemma, which says that knot exteriors can't be "linked" with each other; here, by a nontrivial knot exterior we mean a manifold diffeomorphic to the exterior of a nontrivial knot in $S^{3}$.

Lemma 2.2 Let $Y$ be a manifold and for $i=1, \ldots, p$ let $E_{i}$ be a nontrivial knot exterior embedded in a ball $D_{i} \subset Y$. Suppose that $E_{i} \cap E_{i^{\prime}}=\varnothing$ for $i \neq i^{\prime}$.

Then we may choose the balls $D_{i}$ so that $D_{i} \cap D_{i^{\prime}}=\varnothing$ for $i \neq i^{\prime}$. 
Proof We assume that $E_{i} \cap E_{i^{\prime}}=\varnothing$ during all the isotopies considered in this proof (for $i \neq i^{\prime}$ ). Assume, for a contradiction, that the lemma is false and let $Y$ and $E_{i}$ form a counterexample that minimizes $p$; note that $p \geq 2$. Then there exist disjoint balls $D_{1}, \ldots, D_{p-1}$ such that $E_{i} \subset D_{i}$. Assume first that there is no isotopy of $E_{p}$ such that $E_{p} \cap\left(\bigcup_{i=1}^{p-1} D_{i}\right)=\varnothing$. Let $m_{i} \subset D_{i}$ be a meridian disk for $E_{i}, i=1, \ldots, p-1$; note that $D_{i}$ is isotopic to $E_{i} \cup N\left(m_{i}\right)$. Define

$$
M=\bigcup_{i=1}^{p-1} m_{i} .
$$

Minimize $\left|M \cap E_{p}\right|$; since $E_{p}$ cannot be isotoped to be disjoint from $\bigcup_{i=1}^{p-1} D_{i}$, we have $\left|M \cap E_{p}\right|>0$. Let $\delta \subset M$ be an innermost disk of $M \cap \partial E_{p}$. If $\partial \delta$ is inessential in $\partial E_{p}$, we use an innermost disk from $\partial E_{p}$ to surger $M$ and reduce $\left|M \cap E_{p}\right|$. This gives new (and not necessarily isotopic) meridian disks for $E_{1}, \ldots, E_{p-1}$; we still denote these disks by $m_{1}, \ldots, m_{p-1}$ and $\bigcup_{i=1}^{p-1} m_{i}$ by $M$. We replace the balls $D_{i}$ with $E_{i} \cup N\left(m_{i}\right)$, which we will continue to denote by $D_{i}$. We repeat this process until one of the following holds:

(1) $\left|M \cap E_{p}\right|>0$ and $\partial \delta$ is essential in $\partial E_{p}$ : in that case, $\delta$ is a meridian disk for $E_{p}$. Let $\widehat{D}_{p}=E_{p} \cup N(\delta)$. Then $\widehat{D}_{p} \cap\left(\bigcup_{i=1}^{p-1} E_{i}\right)=\varnothing$. By surgery, we may assume that $M \cap \widehat{D}_{p}=\varnothing$; thus we see that $\left\{D_{i}, \ldots, D_{p-1}, \widehat{D}_{p}\right\}$ is a collection of disjointly embedded balls contradicting our assumption.

(2) $\left|M \cap E_{p}\right|=0$ : in this case $E_{p} \cap\left(\bigcup_{i=1}^{p-1} D_{i}\right)=\varnothing$. Let $m_{p}$ be a meridian disk for $E_{p}$; by surgery we may assume that $m_{p} \cap\left(\bigcup_{i=1}^{p-1} D_{i}\right)=\varnothing$. Then $\left\{D_{i}, \ldots, D_{p-1}, E_{p} \cup N\left(m_{p}\right)\right\}$ is a collection of disjointly embedded balls contradicting our assumption.

Definition 2.3 Let $Y$ be a connected manifold. Let $U \subset Y$ be a link. We say that $U$ is an unlink if the components of $U$ bound disjointly embedded disks.

In the following lemma we consider two links in a manifold $Y$, which we will denote as $\mathcal{L}$ and $\mathcal{U}$. Since $\mathcal{U}$ is an unlink, we can perform $1 / n$ surgery about any component $K$ of $\mathcal{U}$ without changing the ambient manifold; here the framing is chosen so that the boundary of the disk bounded by $K$ corresponds to $0 / 1$. After this surgery say that the components of $\mathcal{L}$ are twisted about $K n$ times. This process may be repeated on the other component of $\mathcal{U}$. We assume that $Y \backslash$ int $N(\mathcal{L} \cup \mathcal{U})$ is irreducible; in Condition (2) below we describe it as $\mathcal{L}$ being irreducible in the complement of $\mathcal{U}$ (that is, $(Y \backslash$ int $N(\mathcal{U})) \backslash$ int $N(\mathcal{L})$ being irreducible). We are interested in the effect on $\mathcal{L}$ when $n$ is large: 
Lemma 2.4 Let $Y$ be a manifold and $\mathcal{L}, \mathcal{U}$ disjoint links in $Y$. Assume that the following conditions hold:

(1) $\mathcal{U}$ is an unlink.

(2) $\mathcal{L}$ is irreducible in the complement of $\mathcal{U}$.

Then the link $\mathcal{L}^{\prime}$ obtained from $\mathcal{L}$ by twisting its components about each component of $\mathcal{U}$ sufficiently many times has an irreducible exterior.

Proof We induct on $|\mathcal{U}|$, the number of components in $\mathcal{U}$. If $|\mathcal{U}|=0$ there is nothing to prove. Otherwise, let $K$ be a component of $\mathcal{U}$. We will denote $Y \backslash \operatorname{int} N(\mathcal{L} \cup \mathcal{U})$ by $X$. Let $\alpha$ be any slope of $\partial N(K)$ such that the manifold obtained by filling $\partial N(K)$ with slope $\alpha$ is reducible. Denote a reducing sphere that minimizes the geometric intersection number with the core of the attached solid torus by $S$. Then it is easy to see that $S_{E}=S \cap Y \backslash$ int $N(\mathcal{L} \cup \mathcal{U})$ is an essential surface with $\partial S_{E} \subset \partial N(K)$ a nonempty collection of essential curves, all defining the slope $\alpha$. By Hatcher [7, Corollary] only finitely many slopes of $\partial N(K)$ bound such a surface. Twisting $n$ times about $K$ is equivalent to filling $\partial N(K)$ with slope $\alpha=1 / n$ (for any choice of basis for $H_{1}(\partial N(K) ; \mathbb{Z})$ for which the boundary of the disk bounded by $K$ corresponds to $0 / 1)$. Thus for all but finitely many values of $n$ the manifold obtained is irreducible. We pick one such $n$, and after twisting $\mathcal{L}$ about $K n$ times, we remove $K$ from $\mathcal{U}$. Induction completes the proof.

The following lemma was proved in [2] by Bleiler, Hodgson and Weeks. It says that the action induced by the mapping class group of $M$ on the slopes on $\partial M$ (which is assumed to be a single torus) is trivial. We bring a new argument here. Our argument holds for many nonhyperbolic manifolds as well: all we require is that $M$ does not admit infinitely many fillings that result in diffeomorphic manifolds; this is well known to hold for hyperbolic manifolds as well as all Seifert fibered spaces except $D^{2} \times S^{1}$. In Lemma 2.5(2) below we use a basis for $H_{1}(\partial M)$ to identify the slopes of $\partial M$ with $\overline{\mathbb{Q}}=\mathbb{Q} \cup\{1 / 0\}$.

Lemma 2.5 Let $M$ be a hyperbolic manifold with $\partial M$ a single torus. Let $\phi: M \rightarrow M$ be an orientation-preserving diffeomorphism. Then for any simple closed curve $\gamma \subset \partial M$, $\gamma$ and $\phi \circ \gamma$ represent the same slope.

Moreover, if $M$ admits orientation-reversing diffeomorphisms, then one of the following holds:

(1) For any orientation-reversing diffeomorphism $\phi$, the curves $\gamma$ and $\phi \circ \gamma$ represent the same slope. 
(2) There is a basis for $H_{1}(\partial M)$ such that, for any orientation-reversing diffeomorphism $\phi$, if a curve $\gamma$ represents the slope $p / q$ then $\phi \circ \gamma$ represents the slope $-p / q$.

Proof We use the notation introduced in the statement of the lemma. By a well-known homological argument we have ker $i_{*} \cong \mathbb{Z}$, where $i_{*}: H_{1}(\partial M ; \mathbb{Z}) \rightarrow H_{1}(M ; \mathbb{Z})$ is the homomorphism induced by the inclusion; moreover, any generator of ker $i_{*}$ is primitive in $H_{1}(\partial M ; \mathbb{Z})$. Let $l$ be a simple curve such that $[l]$ is a generator of ker $i_{*}$ (we denote homology classes by $[\cdot]$ ). Then $[\phi \circ l]= \pm[l]$.

Let $m$ be a simple curve such that $[l]$ and $[m]$ generate $H_{1}(\partial M ; \mathbb{Z})$. Since $\phi$ induces an isomorphism on $H_{1}(\partial M ; \mathbb{Z}),[\phi \circ m]$ and $[\phi \circ l]$ generate $H_{1}(\partial M ; \mathbb{Z})$. Thus $[\phi \circ m]= \pm[m]+n[l]$ for some $n \in \mathbb{Z}$. If $n \neq 0$, the orbit of $[m]$ is infinite. But then the slope represented by $m$ has an infinite orbit, and hence $M$ admits infinitely many fillings (namely, fillings along the slopes represented by $\phi^{k} \circ m$ for $k \in \mathbb{Z}$ ) that produce diffeomorphic manifolds. As $M$ is hyperbolic, this is impossible. Thus $[\phi \circ m]= \pm m$. We will use $[m]$ and $[l]$ as a basis for $H_{1}(\partial M)$. We conclude that $\phi_{*}$ is one of the following maps:

(1) $\phi_{*}((p, q))=(p, q)$.

(2) $\phi_{*}((p, q))=(-p,-q)$.

(3) $\phi_{*}((p, q))=(-p, q)$.

(4) $\phi_{*}((p, q))=(p,-q)$.

Note that cases (3) and (4) imply that $\phi$ is orientation-reversing; hence if $\phi$ is orientationpreserving then $\phi_{*}: H_{1}(\partial M ; \mathbb{Z}) \rightarrow H_{1}(\partial M ; \mathbb{Z})$ is either the identity or the antipodal map; since a slope is defined as the homology class of an unoriented curve, both maps fix all slopes.

If $\phi$ is orientation-reversing and $\phi_{*}((p, q))=(-p, q)$ or $\phi_{*}((p, q))=(p,-q)$, then (using $[l]$ and $[m]$ as a basis for $\left.H_{1}(\partial M ; \mathbb{Z})\right)$ we see that $\phi$ maps the slope $p / q$ to the slope $-p / q$, as required.

All that remains is to show that if $\phi_{1}$ and $\phi_{2}$ are orientation-reversing diffeomorphisms of $M$, then $\phi_{1 *}$ and $\phi_{2 *}$ are both as in cases (1) or (2), or both as in cases (3) or (4). If one is as in cases (1) or (2) and the other as in cases (3) or (4), then $\phi_{2}^{-1} \circ \phi_{1}$ is an orientation-preserving diffeomorphism and $\left(\phi_{2}^{-1} \circ \phi_{1}\right)_{*}$ is as in case (3) or (4), which is impossible. 


\section{D Cores of solid tori}

In this subsection we prove the following necessary condition for a curve in $T^{2} \times[0,1]$ to become a core of the solid torus obtained by filling:

Lemma 2.6 Let $K \subset T^{2} \times[0,1]$ and $c \subset T^{2} \times\{0\}$ be curves, and assume that $c$ is simple and essential. Let $V$ be the solid torus obtained by filling $T^{2} \times\{0\}$ along $c$. Suppose that $[c]$ and $[K]$ do not generate $H_{1}\left(T^{2} \times[0,1] ; \mathbb{Z}\right)$.

Then $K$ is not isotopic to a core of $V$.

Proof We identify $T^{2} \times\{1\}$ with $T^{2}$. We will denote the projections of $c$ and $K$ to $T^{2}$ by $c^{\prime}$ and $K^{\prime}$, respectively. We obtain two classes in $H_{1}\left(T^{2} ; \mathbb{Z}\right)$, defined up to sign, which we will denote by $\pm\left[c^{\prime}\right]$ and $\pm\left[K^{\prime}\right]$.

Suppose that $K$ is isotopic to a core of $V$. Then the signed intersection of $K$ and the meridian disk of $V$ is \pm 1 . Up to isotopy, $c^{\prime}$ is the boundary of the meridian disk of $V$. Therefore, the signed intersection of $K^{\prime}$ with $c^{\prime}$ as curves in $T^{2}$ is \pm 1 (the sign may not agree with that of the intersection of $K$ and the meridian disk of $V$ ). Any class of $H_{1}\left(T^{2} ; \mathbb{Z}\right)$ is represented by $n$ parallel copies of a simple closed curve (possibly $n=0$ ). Let $\gamma$ be a simple closed curve such that $\pm[n \gamma]= \pm\left[c^{\prime}\right]$. Since $c^{\prime}$ intersects $K^{\prime}$ algebraically once, $n= \pm 1$ and we may assume that $\gamma$ and $K^{\prime}$ intersect once. Thus $[\gamma]$ and $\left[K^{\prime}\right]$ generate $H_{1}\left(T^{2} ; \mathbb{Z}\right)$. Since $[\gamma]=\left[c^{\prime}\right]=[c]$ and $\left[K^{\prime}\right]=[K]$, $[c]$ and $[K]$ generate $H_{1}\left(T^{2} \times[0,1] ; \mathbb{Z}\right)$.

\section{E Hyperbolic Dehn surgery}

Let $M$ be a one-cusped hyperbolic manifold. It is well known that, for all but finitely many slopes on $\partial M, M(\beta)$ is hyperbolic and the core of the attached solid torus, which we will denote by $\gamma$, is a geodesic. As we vary $\beta$, the length of the geodesics $\gamma$ obtained satisfies

$$
\lim _{l(\beta) \rightarrow \infty} l(\gamma)=0,
$$

where $l(\beta)$ is measured in the Euclidean metric induced on $\partial M$ after some truncation of the cusp. Moreover, after an appropriate choice of basepoints, as $l(\beta) \rightarrow \infty$, the manifolds $M(\beta)$ converge in Gromov-Hausdorff distance to $M$. With this we obtain the following lemma, which is well known to many experts, but since we could not find a reference we sketch its proof here.

Lemma 2.7 With notation as above, there exists $\epsilon>0$ and a finite set $B_{f}$ of slopes of $\partial M$ such that, for any slope $\beta$ of $\partial M$ for which $\beta \notin B_{f}$, the following three conditions hold: 
(1) $M(\beta)$ is hyperbolic.

(2) $l(\gamma)<\epsilon$.

(3) If $\delta \subset M(\beta)$ is a geodesic and $l(\delta)<\epsilon$, then $\delta=\gamma^{n}$ for some $n$.

Sketch of proof The proofs relies on the thin-thick decomposition which we now review. Fix $\mu>0$. For a hyperbolic manifold $X$, the thick part of $X$, which we will denote by $X_{\geq \mu}$, consists of all the points of $X$ that have radius of injectivity at least $\mu$. The thin part of $X$, which we will denote $X_{\leq \mu}$, is defined to be the closure of the complement of the thick part, that is, $X_{\leq \mu}=\operatorname{cl}\left(X \backslash X_{\geq \mu}\right)$. It is a fundamental fact in hyperbolic geometry that if $\mu$ is small enough (and this is independent of $X$ ) then the thin part consists of normal neighborhoods of the short geodesics and the cusps of $X$. By replacing $\mu$ by a smaller number if necessary we assume that $\mu$ is smaller than half the length of the shortest geodesic of $M$; then $M_{\leq \mu}$ is obtained from $M$ by truncating its cusp.

By the discussion above, (1) and (2) are established in the literature. Assume, for a contradiction, that there does not exist a finite set $B_{f}$ for which (3) holds. Then there is a sequence $\beta_{i}$ of slopes of $\partial M$ with $l\left(\beta_{i}\right) \rightarrow \infty$, and geodesics $\delta_{i} \subset M\left(\beta_{i}\right)$ so that $l\left(\delta_{i}\right)<1 / i$ and $\delta_{i}$ is not a power of $\gamma_{i}$, the core of the attached solid torus. Let $V_{i}$ be the component of $M\left(\beta_{i}\right)_{\leq \mu}$ that contains $\gamma_{i}$. We will denote $N_{1 / 2 i}\left(M\left(\beta_{i}\right) \backslash V_{i}\right)$ by $N_{i}$, where $N_{1 / 2 i}$ denotes the $1 / 2 i$-neighborhood. By construction, $\delta_{i} \subset N_{i}$.

For an appropriate choice of basepoints $x_{i} \in M\left(\beta_{i}\right)$ and $x \in M,\left(M\left(\beta_{i}\right), x_{i}\right)$ converges to $(M, x)$ in the Gromov-Hausdorff sense. Then $f_{i} \circ \delta_{i}$ are closed curves in $M_{\geq \mu}$ with $l\left(f_{i} \circ \delta_{i}\right) \rightarrow 0$. Thus, for sufficiently large $i, l\left(f_{i} \circ \delta_{i}\right)<\mu$, and hence $f_{i} \circ \delta_{i}$ is null homotopic or is homotopic into the cusp. In the former case, let $D \subset M_{\geq \mu}$ be an immersed disk whose boundary is $f_{i} \circ \delta_{i}$. By isotopy we may move $D$ out of the cusp. The image of $D$ under $f_{i}^{-1}$ shows that $\delta_{i}$ bounds an immersed disk, which is a contradiction. In the latter case, we may use an immersed annulus given by the trace of the homotopy of $f_{i} \circ \delta_{i}$ to the cusp to conclude that $\delta_{i}$ is homotopic into a neighborhood of the core of the attached solid torus; this implies that the geodesic $\delta_{i}$ is itself a power of that core.

\section{Bounded sets in the Farey graph}

The Farey graph is a connected graph whose vertices encodes the slopes of the torus; we begin this section with a brief description of this well-known construction; see Figure 1. Viewing each edge as a length-one interval, the Farey graph induces a metric on the slopes which is instrumental for our study: throughout this paper we make 


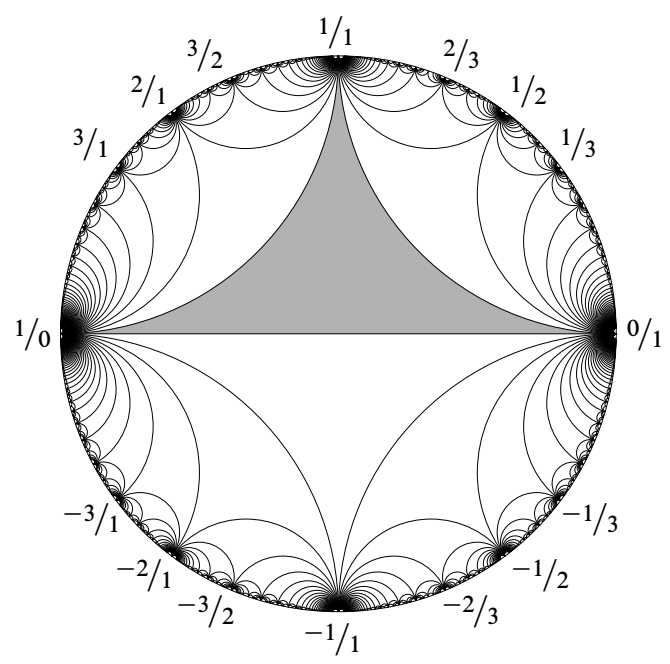

Figure 1: The Farey tessellation

extensive use of sets of slopes that are bounded in this metric. In [19] we noted that any cover between tori induces a bijection on their slopes and argued that branched covers between manifolds can be completed after filling if and only if the slopes filled correspond under this bijection; in Section 3A we recall these facts and prove that this bijection is a bilipschitz map, and hence the image of a bounded set is bounded, and the same holds for preimages. In Section 3B we prove that bounded sets are closed under certain operations, notably Dehn twists and adding slopes of bounded intersection (see Proposition 3.6 for a precise statement). Moreover, we show that bounded sets form the smallest nonempty collection of sets that is closed under these operations; hence, from our point of view, they are the smallest collection of sets we may use.

We now describe the Farey graph; it is best seen as embedded in $\mathbb{H}^{2} \cup S_{\infty}^{1}$ as the 1-skeleton of the Farey tessellation of the hyperbolic plane, although the metric we will use (as described above) is not induced by the hyperbolic metric. For this construction see Figure 1. Pick an ideal triangle in $\mathbb{H}^{2}$ and label its vertices as $0 / 1,1 / 0$, and $1 / 1$. The Farey tessellation is constructed recursively: after reflecting a triangle by an edge whose endpoints are labeled $p / q$ and $r / s$, we obtain a new triangle and label the new vertex by $(p+r) /(q+s)$ or $(p-r) /(q-s)$; the sign depends on the direction of the reflection. This addition rule is induced by the addition in $\mathbb{Z} \times \mathbb{Z}$. At the end of the day we obtain a tessellation of $\mathbb{H}^{2}$ by ideal triangles and it is a well-known consequence of Euclid's algorithm that every element of $\overline{\mathbb{Q}}=\mathbb{Q} \cup\{1 / 0\}$ appears as the label for exactly one vertex. The Farey graph is the graph given by the 1-skeleton of the Farey tessellation. After choosing a basis for $H_{1}\left(T^{2} ; \mathbb{Z}\right)$ we identify the slopes of $T^{2}$ with $\overline{\mathbb{Q}}$. Thus we have a bijection between the slopes of $T^{2}$ and the vertices 
of the Farey tessellation. It is easy to see that the claims below do not depend on the choice of basis, as a change of basis induces an isomorphism of the Farey graphs.

Let $x$ and $y$ be vertices that correspond to slopes $\alpha_{1}$ and $\alpha_{2}$; it is well-known that $x$ and $y$ are connected by an edge in the Farey tessellation if and only if $\alpha_{1}$ and $\alpha_{2}$ can be represented by curves that intersect exactly once. The distance in the Farey tessellation is the minimal number of edges traversed to get from one vertex to another. We define the distance between slopes $\alpha_{1}$ and $\alpha_{2}$ to be the distance between the corresponding vertices of the Farey tessellation and denote it by

$$
d_{\text {Farey }}\left(\alpha_{1}, \alpha_{2}\right) \text {. }
$$

Definition 3.1 A set of slopes is called bounded if it is has bounded diameter using the metric induced by $d_{\text {Farey }}$.

It is well known that the Farey graph has infinite diameter, hence:

Lemma 3.2 If $B$ is a bounded set of slopes then there are infinitely many slopes not in $B$.

\section{A Coverings and slopes}

For this subsection fix tori $T, T^{\prime}$ and a cover $\phi: T \rightarrow T^{\prime}$. In [19] we showed that $\phi$ induces a bijection between the slopes of $T$ and those of $T^{\prime}$; we refer the reader to that paper for a detailed discussion. The correspondence is defined as follows: let $\alpha$ be a slope of $T$ and $\gamma$ an essential connected simple closed curve on $T$ representing $\alpha$. Then $\phi \circ \gamma$ is an essential connected (not necessarily simple) closed curve on $T^{\prime}$, and hence there exist a positive integer $m$ and $\beta^{\prime}$, a connected simple closed curve on $T^{\prime}$ such that $\left(\beta^{\prime}\right)^{m}$ is homologous to $\phi \circ \gamma$. We define the slope represented by $\beta^{\prime}$ to be the slope that corresponds to $\alpha$. Conversely, let $\alpha^{\prime}$ be a slope of $T^{\prime}$ represented by an essential simple closed curve $\gamma^{\prime}$. Then $\phi^{-1}\left(\gamma^{\prime}\right)$ is an essential (not necessarily connected) simple closed curve. We define the slope represented by a component of $\phi^{-1}\left(\gamma^{\prime}\right)$ to be the slope that corresponds to $\alpha^{\prime}$. It is not hard to see that these correspondences are inverses of each other.

We refer to slopes that correspond under this bijection as corresponding slopes. The branched covers between manifolds that we consider in this paper induce covers on boundary components. In [19] we showed that a branched cover $X \rightarrow E$ (where $X$ and $E$ are manifolds with toral boundary and the branch set is closed, that is, disjoint from $\partial E$ ) extends to a branched cover after filling if and only if the slopes filled are corresponding slopes. This simple fact will often be used without reference throughout this paper. 


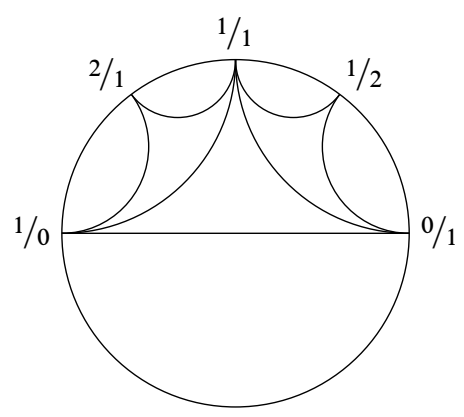

Figure 2: The two quadrilaterals in the Farey tessellation

Remark 3.3 In the example below it will be convenient to take an alternate view of the correspondence between the slopes of $T$ and those of $T^{\prime}$, where $\phi: T \rightarrow T^{\prime}$ is a cover. By lifting $\phi: T \rightarrow T^{\prime}$ appropriately to the universal covers we obtain a matrix in $\operatorname{SL}(2, \mathbb{Q})$. Any such matrix gives a correspondence between lines of rational slope in the universal covers, which are naturally identified with slopes on the tori; the reader can verify that this correspondence is equivalent to the correspondence described above.

Let $T$ and $T^{\prime}$ be tori and $\phi: T \rightarrow T^{\prime}$ a cover as above. We will often consider bounded sets of $T$ or $T^{\prime}$; our goal in this subsection is to understand their behavior under the correspondence induced by $\phi$. We begin with a simple example: consider $T$ and $T^{\prime}$ as $\mathbb{R}^{2} / \sim$, where $\sim$ is given by $(x, y) \sim(x+n, y+m)$ for $n, m \in \mathbb{Z}$. Let $\phi: T \rightarrow T^{\prime}$ be the double cover induced by the map $\tilde{\phi}: \mathbb{R}^{2} \rightarrow \mathbb{R}^{2}$ given by $\tilde{\phi}((x, y))=(x, 2 y)$. The four segments in $\mathbb{R}^{2}$ connecting $(0,0)$ to $(1,0),(2,1),(1,1)$, and $(0,1)$ map to four curves on $T$, defining slopes that can be naturally denoted as $0 / 1,1 / 2,1 / 1$, and $1 / 0$; see Figure 2 . The images of these segments under $\tilde{\phi}$ are the segments connecting $(0,0)$ to $(1,0),(2,2),(1,2)$, and $(0,2)$, and the corresponding slopes are $0 / 1,1 / 1,2 / 1$, and $1 / 0$. Thus the correspondence maps a quadrilateral in the Farey graph of $T$ to a quadrilateral in the Farey graph of $T^{\prime}$; each quadrilateral has exactly one diagonal edge. In the first, the diagonal edge connects $0 / 1$ to $1 / 1$, and in the second it connects $1 / 0$ to $1 / 1$. These edges do not correspond, showing that the correspondence between the slopes of $T$ and those of $T^{\prime}$ (viewed as a bijection between the vertices of the Farey graphs) does not induce an isomorphism of the Farey graphs. Equivalently, the bijection between the slopes of $T$ and $T^{\prime}$ (considered as metric spaces with the metric $d_{\text {Farey }}$ ) is not an isometry. It is easy to see that in this example some distances decrease while others increase. Since we always consider Farey graphs as metric spaces with the metric $d_{\text {Farey }}$, we need the following lemma:

Lemma 3.4 Let $T, T^{\prime}$ be tori and $\phi: T \rightarrow T^{\prime}$ be a cover. Then the correspondence between slopes of $T$ and $T^{\prime}$ is bilipschitz and hence it sends bounded sets of $T$ to bounded sets of $T^{\prime}$ and vice versa. 
Proof For convenience we endow $T^{\prime}$ with a Euclidean metric and $T$ with the pullback metric. Since we are only interested in the homology classes represented by curves, throughout the proof of this lemma we assume as we may that the essential curves of $T$ and $T^{\prime}$ considered are geodesic.

We first show that the map from the slopes of $T$ to those of $T^{\prime}$ is Lipschitz. Fix a positive integer $d$ and two slopes of $T$ of distance exactly $d$, which we will denote by $\alpha_{0}$ and $\alpha_{d}$. By definition of $d_{\text {Farey }}$, there exist slopes $\alpha_{1}, \ldots, \alpha_{d-1}$ such that $d_{\text {Farey }}\left(\alpha_{i-1}, \alpha_{i}\right)=1$ (for $i=1, \ldots, d$ ). Let $\gamma_{0}, \ldots, \gamma_{d}$ be geodesics on $T$ representing $\alpha_{0}, \ldots, \alpha_{d}$, respectively. Since geodesics on a torus minimize intersection, $d_{\text {Farey }}\left(\alpha_{i-1}, \alpha_{i}\right)=1$ is equivalent to $\left|\gamma_{i-1} \cap \gamma_{i}\right|=1$. Let $\gamma_{i}^{\prime}$ be a connected simple closed geodesic on $T^{\prime}$ and $m_{i}$ a positive integer such that $\phi\left(\gamma_{i}\right)$ is obtained by traversing $\gamma_{i}^{\prime}$ exactly $m_{i}$ times. By definition, $\gamma_{i}^{\prime}$ is a geodesic representing $\alpha_{i}^{\prime}$, where $\alpha_{i}^{\prime}$ is the slope of $T^{\prime}$ that corresponds to $\alpha_{i}$. We will denote $\left|\gamma_{i-1}^{\prime} \cap \gamma_{i}^{\prime}\right|$ by $c_{i}$. Let $\Gamma_{i}$ denote $\phi^{-1}\left(\gamma_{i}^{\prime}\right)$ and denote the number of components of $\Gamma_{i}$ by $n_{i}$. Each component of $\Gamma_{i}$ is a geodesic parallel to $\gamma_{i}$ and is an $m_{i}$-fold cover of $\gamma_{i}^{\prime}$; it follows that $n_{i} m_{i}=\operatorname{deg}(\phi)$, and in particular

$$
n_{i} \leq \operatorname{deg}(\phi)
$$

Since $\Gamma_{i-1} \cap \Gamma_{i}=\phi^{-1}\left(\gamma_{i-1}^{\prime} \cap \gamma_{i}^{\prime}\right)$ we have $\left|\Gamma_{i-1} \cap \Gamma_{i}\right|=c_{i} \operatorname{deg}(\phi)$. Since $\left|\gamma_{i-1} \cap \gamma_{i}\right|=1$, every component of $\Gamma_{i-1}$ intersects every component of $\Gamma_{i}$ exactly once; it follows that $\left|\Gamma_{i-1} \cap \Gamma_{i}\right|=n_{i-1} n_{i}$, the number of pairs of curves from $\Gamma_{i-1}$ and $\Gamma_{i}$. Combining these facts, we see that

$$
c_{i} \operatorname{deg}(\phi)=\left|\Gamma_{i-1} \cap \Gamma_{i}\right|=n_{i-1} n_{i} \leq \operatorname{deg}(\phi)^{2},
$$

showing that $c_{i} \leq \operatorname{deg}(\phi)$.

Thus $\Delta\left(\gamma_{i-1}^{\prime}, \gamma_{i}^{\prime}\right) \leq\left|\gamma_{i-1}^{\prime} \cap \gamma_{i}^{\prime}\right| \leq \operatorname{deg}(\phi)$. It then follows from Euclid's algorithm that $d_{\text {Farey }}\left(\alpha_{i-1}^{\prime}, \alpha_{i}^{\prime}\right) \leq 2 \log _{2}(\operatorname{deg}(\phi))$. (For the relation between Euclid's algorithm and the Farey tessellation, see, for example, [6].) We get

$$
\begin{aligned}
d_{\text {Farey }}\left(\alpha_{0}^{\prime}, \alpha_{d}^{\prime}\right) & \leq \sum_{i=1}^{d} d_{\text {Farey }}\left(\alpha_{i-1}^{\prime}, \alpha_{i}^{\prime}\right) \\
& \leq 2 \log _{2}(\operatorname{deg}(\phi)) d \\
& =2 \log _{2}(\operatorname{deg}(\phi)) d_{\text {Farey }}\left(\alpha_{0}, \alpha_{d}\right) .
\end{aligned}
$$

As $d, \alpha_{0}$, and $\alpha_{d}$ were arbitrary we conclude that

$$
d_{\text {Farey }}\left(\alpha_{0}^{\prime}, \alpha_{d}^{\prime}\right) \leq 2 \log _{2}(\operatorname{deg}(\phi)) d_{\text {Farey }}\left(\alpha_{0}, \alpha_{d}\right),
$$


showing that the map from the slopes of $T$ to the slopes of $T^{\prime}$ is Lipschitz with constant $2 \log _{2}(\operatorname{deg}(\phi))$.

The converse is essentially identical and we only sketch it here. Similarly to the argument above, fix $d$ and let $\alpha_{0}^{\prime}, \alpha_{d}^{\prime}$ be slopes of $T^{\prime}$ such that $d_{\text {Farey }}\left(\alpha_{0}^{\prime}, \alpha_{d}^{\prime}\right)=d$. Let $\alpha_{0}^{\prime}, \ldots, \alpha_{d}^{\prime}$ be a sequence of slopes that realizes the distance, that is, $d_{\text {Farey }}\left(\alpha_{i-1}^{\prime}, \alpha_{i}^{\prime}\right)=1$. Let $\gamma_{i}^{\prime}$ be a geodesic on $T^{\prime}$ that represents $\alpha_{i}^{\prime}$; then $\left|\gamma_{i-1}^{\prime} \cap \gamma_{i}^{\prime}\right|=1$. Let $\gamma_{i}$ be a component of $\phi^{-1}\left(\gamma_{i}\right)$. By definition, $\gamma_{i}$ represents $\alpha_{i}$, the slope of $T$ that corresponds to $\alpha_{i}^{\prime}$. Since $\gamma_{i-1} \cap \gamma_{i} \subset \phi^{-1}\left(\gamma_{i-1}^{\prime} \cap \gamma_{i}^{\prime}\right)$ we have

$$
\left|\gamma_{i-1} \cap \gamma_{i}\right| \leq\left|\phi^{-1}\left(\gamma_{i-1}^{\prime} \cap \gamma_{i}^{\prime}\right)\right|=\operatorname{deg}(\phi)\left|\gamma_{i-1}^{\prime} \cap \gamma_{i}^{\prime}\right|=\operatorname{deg}(\phi) .
$$

As above, this implies that $d_{\text {Farey }}\left(\alpha_{i-1}, \alpha_{i}\right) \leq 2 \log _{2}(\operatorname{deg}(\phi))$, and hence

$$
d_{\text {Farey }}\left(\alpha_{0}, \alpha_{d}\right) \leq 2 \log _{2}(\operatorname{deg}(\phi)) d_{\text {Farey }}\left(\alpha_{0}^{\prime}, \alpha_{d}^{\prime}\right),
$$

showing that the map from the slopes of $T^{\prime}$ to the slopes of $T$ is Lipschitz with constant $2 \log _{2}(\operatorname{deg}(\phi))$.

\section{B On bounded sets}

In this subsection we study properties of bounded sets, that is, sets of slopes that are bounded in the metric $d_{\text {Farey }}$ (as defined in Definition 3.1). Our main goal is to show (Proposition 3.6) that bounded sets are closed under certain operations, the most important of which is Dehn twists, which we explain below. We then note (Lemma 3.7) that any nonempty collection of sets of slopes that is closed under the operations discussed in Proposition 3.6 contains all bounded sets; therefore boundedness is the weakest condition that suffices for our work.

Let $\alpha$ be a slope on $T$ represented by a connected simple closed curve $\gamma$. By definition $\alpha$ determines $\gamma$ up to isotopy. Hence the Dehn twist about $\gamma$ is completely determined by $\alpha$. The Dehn twist about $\gamma$ induces a map on the slopes of $T$, which we will denote as $D_{\alpha}$. Although the proper way to refer to $D_{\alpha}$ is "the map induced on the slopes of $T$ by Dehn twisting about a simple connected curve representing $\alpha$ " we will, for simplicity's sake, refer to it as the Dehn twist about $\alpha$.

Since any Dehn twist is a homeomorphism of $T$, it sends any pair of curves that intersect once to a pair of curves that intersect once; hence, for any slope $\alpha, D_{\alpha}$ induces a graph isomorphism of the Farey graph, and in particular $D_{\alpha}$ induces an isometry on the slopes of $T$ with the metric given by $d_{\text {Farey }}$. Before stating our next proposition we introduce the following notation, which will be used throughout this paper: 
Notation 3.5 Let $T$ be a torus. For slopes $\alpha, \beta$ of $T$ we will use the notation $\Delta(\alpha, \beta)$ to denote the geometric intersection number between $\alpha$ and $\beta$. Given a set of slopes $B$ and a positive integer $c$, we will denote by $\Delta(B, c)$ the set of slopes that intersect some member of $B$ at most $c$ times; that is,

$$
\Delta(B, c)=\{\alpha \mid \Delta(\alpha, \beta) \leq c \text { for some } \beta \in B\} .
$$

We are now ready to state:

Proposition 3.6 Let $T^{2}$ be a torus. The following conditions hold for sets of slopes on $T^{2}$ :

(1) A subset of a bounded set is bounded.

(2) Finite unions of bounded sets are bounded.

(3) For any slope $\alpha$ and any bounded set of slopes $B$, the set

$$
\bigcup_{n \in \mathbb{Z}}\left\{D_{\alpha}^{n}(\beta) \mid \beta \in B\right\}
$$

is bounded (where $D_{\alpha}$ is the Dehn twist about $\alpha$ as defined above).

(4) For any integer $c \geq 0$ and any bounded set $B$, the set of slopes $\Delta(B, c)$ is bounded.

Proof (1) Obvious from the definition of a metric space.

(2) Obvious from the definition of a metric space.

(3) Since $D_{\alpha}$ is induced by a homeomorphism of $T^{2}$, it is clearly an isometry of the Farey graph; moreover, $\alpha$ is fixed under $D_{\alpha}$. Let $d>0$ be the diameter of $B$; fix $\beta \in B$. By the triangle inequality, for any $n_{1}, n_{2} \in \mathbb{Z}$ and any $\beta_{1}, \beta_{2} \in B$ we have

$$
\begin{aligned}
d_{\text {Farey }}\left(D_{\alpha}^{n_{1}}\left(\beta_{1}\right), D_{\alpha}^{n_{2}}\left(\beta_{2}\right)\right) \leq d_{\text {Farey }}( & \left.D_{\alpha}^{n_{1}}\left(\beta_{1}\right), D_{\alpha}^{n_{1}}(\beta)\right)+d_{\text {Farey }}\left(D_{\alpha}^{n_{1}}(\beta), \alpha\right) \\
& +d_{\text {Farey }}\left(\alpha, D_{\alpha}^{n_{2}}(\beta)\right)+d_{\text {Farey }}\left(D_{\alpha}^{n_{2}}(\beta), D_{\alpha}^{n_{2}}\left(\beta_{2}\right)\right) .
\end{aligned}
$$

Since $\alpha$ is fixed under the isometry $D_{\alpha}^{n_{1}}$, for the second term we have

$$
d_{\text {Farey }}\left(D_{\alpha}^{n_{1}}(\beta), \alpha\right)=d_{\text {Farey }}\left(D_{\alpha}^{n_{1}}(\beta), D_{\alpha}^{n_{1}}(\alpha)\right)=d_{\text {Farey }}(\beta, \alpha) .
$$

Similarly, the third term is bounded above by $d_{\text {Farey }}(\beta, \alpha)$. For the first term we have

$$
d_{\text {Farey }}\left(D_{\alpha}^{n_{1}}\left(\beta_{1}\right), D_{\alpha}^{n_{1}}(\beta)\right) \leq d_{\text {Farey }}\left(\beta_{1}, \beta\right) \leq d .
$$

Similarly, the fourth term is bounded by $d$. Combining these bounds, we see that the set $\bigcup_{n \in \mathbb{Z}}\left\{D_{\alpha}^{n}(\beta) \mid \beta \in B\right\}$ is bounded with diameter at most

$$
2 d+2 d_{\text {Farey }}(\alpha, \beta) \text {. }
$$


(4) We first prove the claim when $B$ has only one element, which we will denote by $\beta$. By an appropriate choice of basis for $H_{1}\left(T^{2} ; \mathbb{Z}\right)$ we may identify the slopes of $T$ with $\mathbb{Q} \cup\{1 / 0\}$ so that $\beta$ corresponds to $1 / 0$. It is well known that for any slope $p / q$, $d_{\text {Farey }}(1 / 0, p / q)$ is exactly the length of the shortest continued fraction expansion of $p / q$ (see, for example, [21]). On the other hand, $\Delta(1 / 0, p / q)=\left|\operatorname{det}\left(\begin{array}{ll}1 & p \\ 0 & q\end{array}\right)\right|=|q|$. Thus the slopes under consideration correspond to $p / q$ with $|q| \leq c$. By a well-known application of Euclid's algorithm, such a number has a continued fraction expansion of length at most $2 \log _{2}(|q|)$. Thus every slope in $B$ has distance at most $2 \log _{2}(|q|)$ from $\beta$, showing that $B$ is bounded with diameter at most $4 \log _{2}(c)$.

For the general case, let $B$ be a bounded set of slopes and $c \geq 0$ an integer. We will denote the diameter of $B$ by $d$. For $\alpha_{1}, \alpha_{2} \in \Delta(B, c)$, let $\beta_{1}, \beta_{2} \in B$ be slopes such that $\Delta\left(\alpha_{1}, \beta_{1}\right) \leq c$ and $\Delta\left(\alpha_{2}, \beta_{2}\right) \leq c$. Then the following two inequalities hold:

- $d_{\text {Farey }}\left(\alpha_{1}, \beta_{1}\right), d_{\text {Farey }}\left(\alpha_{2}, \beta_{2}\right) \leq 4 \log _{2}(c)$,

- $d_{\text {Farey }}\left(\beta_{1}, \beta_{2}\right) \leq d$.

(The first point follows from the argument above and the second from the fact that the diameter of $B$ is $d$.) By the triangle inequality, $\Delta(B, c)$ is bounded with diameter at $\operatorname{most} 4 \log _{2}(c)+d$.

The reader may wonder if "bounded sets" is the right concept to use. First we give an example that will explain why using unbounded sets may be tricky. Using the upper half plane model of $\mathbb{H}^{2}$, we construct the Farey tessellation starting with the triangle 0,1 and $1 / 0$ (the point at infinity). Then the slopes are naturally identified with $\mathbb{Q} \cup 1 / 0$; this identification is used throughout this example. Let $q_{i}^{\prime}$ be an enumeration of $\mathbb{Q} \cap[0,1)$, $i=1,2, \ldots$. Let $D_{1 / 0}$ denote the map on the slopes induced by Dehn twist about $1 / 0$; it is not hard to see that for any slope $\alpha \neq 1 / 0$ we have $D_{1 / 0}(\alpha)=\alpha+1$. Let $q_{i}=D^{i}\left(q_{i}^{\prime}\right)$. Finally, let $S=\left\{q_{i}\right\}$. Now $S$ is a fairly "thin" set of slopes; it has only one member in every interval $[n, n+1), n=1,2, \ldots$, and so its only accumulation point is $1 / 0$. However, if we allow twisting about $1 / 0$, we see the following: for any slope $\alpha \neq 1 / 0$, there is a unique $j \in \mathbb{Z}$ such that $\alpha+j \in[0,1)$. Hence, there is a unique $i$ such that $\alpha+j=q_{i}^{\prime}$. Thus

$$
\alpha=D_{1 / 0}^{-j}\left(q_{i}^{\prime}\right)=D_{1 / 0}^{-(i+j)}\left(q_{i}\right) .
$$

In other words, $\left\{D_{1 / 0}^{n}\left(q_{i}\right) \mid q_{i} \in S, n \in \mathbb{Z}\right\}$ is the set of all slopes but $1 / 0$. As we shall see below repeatedly, when considering cosmetic surgery one must allow for Dehn twists; thus, the analogue of Proposition 3.6 for the set $S$ is very much false, and such a set cannot be used in our work. 
On the other hand we have the following lemma, that tells us that all bounded sets must be permitted; in that sense, our results cannot be improved; here $\mathcal{P}(S)$ stands for the power set (that is, set of all subsets) of $S$. Note that the assumptions of the lemma below are, essentially, weak versions of the assumption of Proposition 3.6. For assumption (c), recall Notation 3.5.

Lemma 3.7 Let $S$ denote the set of all slopes and suppose $Z \subset \mathcal{P}(S)$ satisfies the following conditions:

(a) $Z$ contains a nonempty set.

(b) $Z$ is closed under subsets.

(c) For any set $B \in Z$, we have that $\Delta(B, 1) \in Z$.

Then $Z$ contains all the bounded sets.

Proof Let $B \in Z$ denote a nonempty set; $B$ exists by assumption (a). Let $\alpha$ be a slope in $B$. For a nonnegative integer $d$, let $B(\alpha, d) \subset S$ be the set of all slopes of distance at most $d$ from $\alpha$, that is,

$$
B(\alpha, d)=\left\{\alpha^{\prime} \in S \mid d_{\text {Farey }}\left(\alpha, \alpha^{\prime}\right) \leq d\right\} .
$$

We claim that for any nonnegative integer $d$ we have that $B(\alpha, d) \in Z$. The proof is an induction on $d$. For the base case $d=0$ we have that $B(\alpha, 0)=\{\alpha\}$. Since $\alpha \in B$ and $Z$ is closed under subsets (assumption (b)), we have that $\{\alpha\} \in Z$.

For the inductive step fix $d>0$. By the induction assumption, $B(\alpha, d-1) \in Z$. Let $\alpha^{\prime} \in B(\alpha, d) \backslash B(\alpha, d-1)$. Then there is some $\alpha^{\prime \prime} \in B(\alpha, d-1)$ such that $d_{\text {Farey }}\left(\alpha^{\prime}, \alpha^{\prime \prime}\right)=1$. By definition of the metric on the Farey graph, this is equivalent to $\Delta\left(\alpha^{\prime}, \alpha^{\prime \prime}\right)=1$. Hence $\alpha^{\prime} \in \Delta(B(\alpha, d-1), 1)$. We see that $B(\alpha, d) \subset \Delta(B(\alpha, d-1), 1)$. By assumption (c) we have that $\Delta(B(\alpha, d-1), 1) \in Z$ and by assumption (b) we have that $B(\alpha, d) \in Z$, as required.

Next let $B$ be an arbitrary nonempty bounded set. We will denote the diameter of $B$ by $d_{B}$. We fix a slope of $B$, which we will denote by $\alpha_{B}$. Then for any slope $\alpha_{B}^{\prime} \in B$, by the triangle inequality we have that

$$
d_{\text {Farey }}\left(\alpha, \alpha_{B}^{\prime}\right) \leq d_{\text {Farey }}\left(\alpha, \alpha_{B}\right)+d_{\text {Farey }}\left(\alpha_{B}, \alpha_{B}^{\prime}\right) \leq d_{\text {Farey }}\left(\alpha, \alpha_{B}\right)+d_{B} .
$$

Hence $B \subset B\left(\alpha, d_{\text {Farey }}\left(\alpha, \alpha_{B}\right)+d_{B}\right)$, and by assumption (b) we have that $B \in Z$.

We conclude this section by revisiting Lemma 2.5: 
Lemma 3.8 Let $M$ be a hyperbolic manifold, $\partial M$ a single torus. There exists a (possibly trivial) involution $i$ on the set of slopes of $\partial M$ that induces an isometry on the Farey graph, so that if $\phi: M \rightarrow M$ is a diffeomorphism and $\alpha$ a slope of $\partial M$, the image of $\alpha$ under $\phi$ is either $\alpha$ or $i(\alpha)$.

Proof This follows immediately from Lemma 2.5 and the fact that $(p, q) \mapsto(-p, q)$ induces an isometric involution on the Farey graph (namely, the isometry given by the reflection by the edge connecting $1 / 0$ to $0 / 1$, and the endpoints of this edge are its fixed slopes).

\section{Minimally nonhyperbolic fillings}

From this section on, the notation introduced in Section 2A will be used regularly; we assume the reader is comfortable with it. In this section we study minimally nonhyperbolic fillings, a concept which is designed for studying exceptional surgeries on links with multiple components, or equivalently, exceptional fillings on manifolds with multiple boundary components. There are two reasons for studying minimally nonhyperbolic fillings: first, every nonhyperbolic filling admits a minimally nonhyperbolic partial filling; second, in Proposition 4.2 we show that any manifold admits only finitely many minimally nonhyperbolic fillings. This is essential for finiteness of $T(X)$, the tree that we will construct in the next section.

Let us begin with a simple example. Let $X$ be the exterior of the Whitehead link endowed with the natural meridian and longitude on each boundary component. For $j=$ $1,2, \ldots$, let $\left(\alpha_{1}^{j}, \alpha_{2}^{j}\right)=\left(1 / 0, p^{j} / q^{j}\right)$ for some $p^{j}, q^{j}$. Then for each $j, X\left(\alpha_{1}^{j}, \alpha_{2}^{j}\right)$ is a lens space and hence $\left(\alpha_{1}^{j}, \alpha_{2}^{j}\right)$ is a nonhyperbolic multislope. There is no mystery here: all the multislopes $\left(\alpha_{1}^{j}, \alpha_{2}^{j}\right)$ have a common partial filling, namely $(1 / 0, \infty)$, and $X(1 / 0, \infty) \cong D^{2} \times S^{1}$ is nonhyperbolic. In this situation, it is better to consider the single multislope $(1 / 0, \infty)$ and not the infinite set of multislopes $\left\{\left(\alpha_{1}^{j}, \alpha_{2}^{j}\right)\right\}_{j=1}^{\infty}$. This leads us to the following definition, which is central to our work:

Definition 4.1 (minimally nonhyperbolic filling) Let $X$ be a hyperbolic manifold, $\mathcal{T}=T_{1} \cup \cdots \cup T_{n}$ components of $\partial X$, and $\alpha$ a multislope of $\mathcal{T}$. We say that $\alpha$ is minimally nonhyperbolic if $X(\alpha)$ is nonhyperbolic and any strict partial filling $\alpha^{\prime} \subset_{\text {pf }} \alpha$ is hyperbolic.

So, for instance, in the example of the Whitehead link exterior discussed above, the slopes $\left(1 / 0, p^{j} / q^{j}\right)$ are not minimally nonhyperbolic and $(1 / 0, \infty)$ is.

We prove: 
Proposition 4.2 Let $X$ be a hyperbolic manifold and $\mathcal{T}=T_{1} \cup \cdots \cup T_{n}$ a union of components of $\partial X$. Then there are only finitely many minimally nonhyperbolic fillings on $\mathcal{T}$.

Proof Since a minimally nonhyperbolic filling is, in particular, a multislope that yields a nonhyperbolic manifold, we assume as we may that there are infinitely many multislopes on $\mathcal{T}$ yielding nonhyperbolic manifolds. Let $\alpha^{j}=\left(\alpha_{1}^{j}, \ldots, \alpha_{n}^{j}\right)$ be an infinite set of distinct nonhyperbolic fillings $(j=1,2, \ldots)$. We will show that one of these slopes is not minimally nonhyperbolic. Note that the proposition will follow by contradiction: if $X$ admitted infinitely many minimally nonhyperbolic fillings, we could take $\alpha^{j}=\left(\alpha_{1}^{j}, \ldots, \alpha_{n}^{j}\right)$ to be that set.

After subsequencing $n$ times we may assume that for each $1 \leq i \leq n, \alpha_{i}^{j}$ (the restrictions of $\alpha^{j}$ to $T_{i}$ ) consists of slopes that are either all distinct or all the same, that is, for each $i$ exactly one of the following holds:

(1) $\alpha_{i}^{j} \neq \alpha_{i}^{j^{\prime}}$ for $j \neq j^{\prime}$; we assume in addition that $\alpha_{i}^{j} \neq \infty$ for any $j$.

(2) $\alpha_{i}^{j}=\alpha_{i}^{j^{\prime}}$ for any $j, j^{\prime}$ (possibly $\alpha_{i}^{j}=\infty$ ).

To avoid overly complicated notation we do not rename $\alpha^{j}=\left(\alpha_{1}^{j}, \ldots, \alpha_{n}^{j}\right)$. After reordering the boundary components if necessary, we may assume that $\alpha_{i}^{j}$ are distinct for $1 \leq i \leq k$, and $\alpha_{i}^{j}$ is constant for $k+1 \leq i \leq n$ (for some $0 \leq k \leq n$ ). Note that $k=0$ is impossible since $\left\{\alpha^{j}\right\}$ is infinite. We drop the superscript from $\alpha_{i}$ for $i>k$.

Let $\hat{X}=X\left(\infty, \ldots, \infty, \alpha_{k+1}, \ldots, \alpha_{n}\right)$. Then the manifolds

$$
\hat{X}\left(\alpha_{1}^{j}, \ldots, \alpha_{k}^{j}\right)=X\left(\alpha_{1}^{j}, \ldots, \alpha_{n}^{j}\right)
$$

are all nonhyperbolic by assumption. We claim that $\hat{X}$ is not hyperbolic. Assume, for a contradiction, that $\hat{X}$ is hyperbolic. Since $\alpha_{i}^{j} \rightarrow \infty$ for all $i$, by Thurston's Dehn surgery theorem, for large enough $j, \hat{X}\left(\alpha_{1}^{j}, \ldots, \alpha_{k}^{j}\right)$ would be hyperbolic as well. This contradicts our assumptions and shows that $\hat{X}$ is not hyperbolic. Recall that $k \geq 1$, and that $\alpha_{i} \neq \infty$ for $1 \leq i \leq k$; hence the multislope $\left(\infty, \ldots, \infty, \alpha_{k+1}, \ldots, \alpha_{n}\right)$ is a nonhyperbolic strict partial filling of $\left(\alpha_{1}^{j}, \ldots, \alpha_{k}^{j}, \alpha_{k+1}, \ldots, \alpha_{n}\right)$, showing that the latter are not minimally nonhyperbolic (for any $j$ ). The proposition follows.

\section{$5 \quad T(X)$}

In this section we construct the tree $T(X)$, which is the main tool for our work on cosmetic surgery. The construction relies heavily on the concept of minimally 
nonhyperbolic fillings from the previous section. After constructing $T(X)$ we prove (Proposition 5.1) that it is finite. We then explain (Proposition 5.2) why $T(X)$ can be used to study fillings. Since $T(X)$ was designed for studying exceptional surgeries on hyperbolic manifolds, it is perhaps a little surprising that it can also be used to study hyperbolic fillings; this is explained and proved in Proposition 5.4.

Let $X$ be a compact orientable manifold whose boundary consists of tori and $\mathcal{T}=$ $T_{1} \cup \cdots \cup T_{n}$ a union of components of $\partial X$. We wish to associate to $(X, \mathcal{T})$ a finite rooted tree, denoted $T(X, \mathcal{T})$ (or $T(X)$ when no confusion can arise), that encodes exceptional fillings on $X$ along the components $\mathcal{T}$.

Before constructing $T(X)$ we comment on its structure. The vertices of $T(X)$ correspond to manifolds with $X$ as the root. We direct every edge away from the root. Branches, which are always assumed to follow this direction, encode the filling process, and therefore we may have distinct vertices that correspond to diffeomorphic manifolds. It follows from the construction below that the vertices are arranged along levels. The levels are grouped into blocks of the form $3 m, 3 m+1$, and $3 m+2$ and obey the following rules:

(1) Geometric manifolds (that is, hyperbolic manifolds, Seifert manifolds, and sol manifolds) are arranged on levels $3 m$ (for $m \in \mathbb{Z}_{\geq 0}$ ).

(2) Reducible manifolds are arranged on levels $3 m+1$ (for $m \in \mathbb{Z}_{\geq 0}$ ).

(3) JSJ manifolds (recall Definition 2.1) are arranged on levels $3 m+2$ (for $m \in \mathbb{Z}_{\geq 0}$ ).

(4) Every edge in $T(X)$ is directed from the initial vertex to the terminal vertex (say from $u$ to $v$ ) so that if $u$ is at level $3 m, 3 m+1$, or $3 m+2$, then $v$ is at level $3 m+1,3 m+2$, or $3 m+3$; moreover, the level of $v$ is strictly greater than that of $u$. We call $u$ the predecessor of $v$ and $v$ the direct descendant of $u$.

We are now ready to construct $T(X)$. The root of $T(X)$ is a vertex labeled $X$. Assume first that $X$ is geometric. Then $X$ is placed in level 0 . If $X$ is Seifert fibered or a sol manifold, then the corresponding vertex is an isolated vertex: there are no edges out of $X$. If $X$ is hyperbolic, we place one edge $e$ out of $X$ for each minimally nonhyperbolic filling on $X$ (say $\alpha$ ), and the terminal vertex of $e$ is labeled $X(\alpha)$. Since $\alpha$ is a nonhyperbolic filling, $X(\alpha)$ is one of the following:

(1) Reducible: then $X(\alpha)$ is placed at level 1 .

(2) JSJ: then $X(\alpha)$ is placed at level 2 .

(3) Seifert fibered or sol manifold: then $X(\alpha)$ is placed at level 3. 
Next suppose that $X$ is reducible. We emphasize that when $X$ is irreducible but not prime - that is, when $X \cong S^{2} \times S^{1}$ — we consider it a Seifert fibered manifold and place it at level 3 with no descendants. Then the corresponding vertex is placed at level 1. Let $X_{1}, \ldots, X_{k}$ be the factors of the prime decomposition of $X$. We place $k$ edges out of $X$ with terminal vertices labeled $X_{1}, \ldots, X_{k}$. Each $X_{i}$ is either JSJ, hyperbolic, Seifert fibered, or sol. Accordingly, the corresponding vertex is placed at level 2 (if it is JSJ) or 3 (in all other cases). Note that if $i \neq i^{\prime}$ then $X_{i}$ and $X_{i^{\prime}}$ correspond to distinct vertices even if $X_{i} \cong X_{i^{\prime}}$.

Finally, let $X$ be a JSJ manifold. The corresponding vertex is placed at level 2. Let $X_{1}, \ldots, X_{k}$ be the components of the torus decomposition of $X$. We place $k$ edges out of $X$, with terminal vertices labeled $X_{1}, \ldots, X_{k}$. Each $X_{i}$ is hyperbolic or Seifert fibered. Appropriately, they are all placed at level 3. As above, if $i \neq i^{\prime}$ then $X_{i}$ and $X_{i^{\prime}}$ correspond to distinct vertices even if $X_{i} \cong X_{i^{\prime}}$.

The construction is recursive, and if $X_{1}$ is a direct descendant of $X$, then we place $T\left(X_{1}\right)$ with the root at the vertex labeled $X_{1}$; since $X_{1}$ is a direct descendant of $X$ its level is 1,2 or 3 . If the level is 3 we shift all the levels in $T\left(X_{1}\right)$ by +3 .

Let us discuss an example. Let $X_{3}$ be a hyperbolic manifold with $m+1$ boundary components. Let $X_{2}$ be double of $X_{3}$ along $m$ boundary components. Hence $X_{2}$ is a toroidal manifold with two boundary components. Let $X_{0}$ be the manifold obtained from $X_{2}$ by drilling a hyperbolic knot (which is known to exist, essentially by Myers [16, Corollary 6.3]). Hence $X_{0}$ is a hyperbolic manifold with three boundary components. Now in $T\left(X_{0}\right)$ there is an edge connecting $X_{0}$ to $X_{2}$, corresponding to a minimally nonhyperbolic filling (since we fill only one boundary component, the filling must be minimal). Next we see two edges from $X_{2}$ to two copies of $X_{3}$. Thus as we move down $T\left(X_{0}\right)$ we start with a single hyperbolic manifold with three boundary components, and later encounter two hyperbolic manifolds, each with $m+1$ boundary components for an arbitrary $m$ (and, perhaps, other manifolds as well — this may not be all the edges between the levels 0 and 3$)$. As the tree of $T\left(X_{0}\right)$ contains two copies of $T\left(X_{3}\right)$ it can be quite big. We leave it as an exercise to the reader to construct other complicated examples; for instance, given an integer $m$, construct a hyperbolic manifold $X$ with one boundary component such that $T(X)$ admits a directed path of length $m$.

Our goal is to use $|T(X)|$, the number of vertices in $T(X)$, as a basis for induction. For that we need:

Proposition 5.1 $T(X)$ is finite. 
Proof Let the degree of a vertex $v$ be the number of direct descendants of $v$ (that is, the number of edges out of $v$ ). It is easy to see that the degree of every vertex $v$ is finite:

(1) If $v$ corresponds to a hyperbolic manifold $X$, the degree is finite because $X$ admits only finitely many minimally nonhyperbolic fillings (Proposition 4.2).

(2) If $v$ corresponds to a Seifert manifold or a sol manifold the degree is zero by construction.

(3) If $v$ corresponds to a reducible manifold the degree is the number of factors in its prime decomposition and is therefore finite.

(4) If $v$ corresponds to a JSJ manifold the degree is finite by the finiteness of the torus decomposition [10] and [11].

The problem is avoiding an infinite branch. Assume there is such a branch. Now by construction every edge starting on level $3 m, 3 m+1$, or $3 m+2$ ends at a level $3 m+1,3 m+2$, or $3 m+3$. The vertices at level $3 m+3$ that do not correspond to hyperbolic manifolds are leaves; hence the branch must admit a vertex corresponding to a hyperbolic manifold on every level $3 m$. Let $X_{3 m}$ denote this hyperbolic manifold.

We will use the Gromov norm; for the definition see [5]. The Gromov norm has the following properties, proved in [5] and [22, Theorem 1] (here $X$ is a compact orientable manifold such that $\partial X$ consists of tori):

(1) If the Gromov norm is nonzero, then it strictly decreases under any filling.

(2) The Gromov norm of $X$ equals the sum of the Gromov norms of the components of the prime decomposition of $X$.

(3) The Gromov norm is additive under decomposition along essential tori.

(4) The Gromov norm is additive under disjoint union.

(5) The Gromov norm is nonnegative.

Since $X_{3 m+3}$ is obtained from $X_{3 m}$ by filling, then (possibly) reducing along essential spheres and discarding components, and (possibly) decomposing along essential tori and discarding components, we see that the Gromov norm of $X_{3 m+3}$ is strictly smaller than that of $X_{3 m}$. It is well known that the hyperbolic volume is a constant multiple of the Gromov norm, and so we see that $X_{0}, X_{3}, X_{6}, \ldots$ forms a sequence of hyperbolic manifolds with $\operatorname{Vol}\left(X_{0}\right)>\operatorname{Vol}\left(X_{3}\right)>\operatorname{Vol}\left(X_{6}\right)>\cdots$. But this cannot be, as hyperbolic volumes are well ordered. 
In the following sections, we will use $T(X)$ inductively. The problem is that we are only dealing with filling, while reducible and JSJ manifolds are treated differently on $T(X)$ (decomposition along spheres and essential tori, respectively). Let $\alpha=\left(\alpha_{1}, \ldots, \alpha_{n}\right)$ be a multislope. If there exists a minimally nonhyperbolic filling $\alpha^{\prime}$ such that $\alpha^{\prime} \subset_{\text {pf }} \alpha$ (possibly $\alpha^{\prime}=\alpha$ ) we say that $\alpha$ admits a minimally nonhyperbolic partial filling. Note that if $\alpha$ is a nonhyperbolic multislope, which by definition means that $X$ is hyperbolic and $X(\alpha)$ is not, then $\alpha$ admits a minimally nonhyperbolic partial filling.

We will need the following proposition; it is useful, for example, when $\alpha$ is itself a hyperbolic multislope, but admits a minimally nonhyperbolic partial filling (note that such multislopes do exist; constructing examples is quite easy).

Proposition 5.2 Let $X$ be a hyperbolic manifold and $\alpha$ a multislope of $\partial X$. Suppose that $\alpha$ admits a minimally nonhyperbolic partial filling $\alpha^{\prime}$ so that $X\left(\alpha^{\prime}\right)$ is either reducible or JSJ. Suppose further that $X(\alpha)$ is irreducible and atoroidal.

Then $X(\alpha)$ is obtained by filling some direct descendant of $X\left(\alpha^{\prime}\right)$ on $T(X)$.

Remark 5.3 The theorem allows for the possibility that $X(\alpha)$ is itself a direct descendant of $X\left(\alpha^{\prime}\right)$. In that case, the theorem simply says that $X(\alpha)$ is obtained by filling $X(\alpha)$ along the trivial multislope $(\infty, \ldots, \infty)$.

Proof Since $\alpha^{\prime} \subset_{\mathrm{pf}} \alpha$, it is clear that $X(\alpha)$ is obtained from $X\left(\alpha^{\prime}\right)$ by filling.

Assume first that $X\left(\alpha^{\prime}\right)$ is reducible. Then the descendants of $X\left(\alpha^{\prime}\right)$ are, by construction of $T(X)$, the factors of the prime decomposition of $X\left(\alpha^{\prime}\right)$. Since $X(\alpha)$ is a prime manifold that is obtained from $X\left(\alpha^{\prime}\right)$ by filling, it is easy to see that in that filling all the components in the prime decomposition of $X\left(\alpha^{\prime}\right)$ become $S^{3}$ except at most one. The proposition follows in this case.

Next assume that $X\left(\alpha^{\prime}\right)$ is JSJ. By assumption, $X(\alpha)$ is prime and atoroidal. Therefore every torus $T$ in $X(\alpha)$ fulfills at least one of the following three conditions:

(1) $T$ is boundary parallel.

(2) $T$ bounds a solid torus.

(3) $T$ bounds a knot exterior contained in a ball.

Since $X(\alpha)$ is obtained from $X\left(\alpha^{\prime}\right)$ by Dehn filling, $X\left(\alpha^{\prime}\right) \subset X(\alpha)$. Let $T \subset X\left(\alpha^{\prime}\right)$ be a torus. Considering $T$ as a torus in $X(\alpha)$ allows us to endow it with a co-orientation as follows: 
(1) If $T$ is boundary parallel, we co-orient it towards the boundary.

(2) If $T$ bounds a solid torus, we co-orient it towards the solid torus.

(3) If $T$ bounds a knot exterior contained in a ball, we co-orient it towards the knot exterior.

Note that some tori may get both co-orientations (for example, a boundary parallel torus in a solid torus or an unknotted torus in a ball). In that event we pick an orientation arbitrarily.

Let $\mathcal{T}$ be the tori of the JSJ decomposition of $X\left(\alpha^{\prime}\right)$. Let $\Gamma$ be the graph dual to $\mathcal{T}$ (recall that in Definition 2.1 we defined the graph dual to $\mathcal{T}$ to be the graph that has one vertex for each component of the torus decomposition of $X\left(\alpha^{\prime}\right)$ and one edge for each torus $T \in \mathcal{T}$, where the edge corresponding to $T$ connects the vertices that correspond to the components adjacent to $T$ ). Since $X$ is connected so is $X\left(\alpha^{\prime}\right)$; hence $\Gamma$ is connected. Since $X(\alpha)$ is atoroidal and irreducible it contains no nonseparating tori, and is follows that neither does $X\left(\alpha^{\prime}\right)$; hence $\Gamma$ contains no cycles. We conclude that $\Gamma$ is a tree. We endow each edge of $\Gamma$ with an orientation consistent with the co-orientation of the corresponding torus of $\mathcal{T}$. Using induction, it is easy to see that $\Gamma$ admits a source (a vertex connected only to edges that point away from it): since $\mathcal{T} \neq \varnothing, \Gamma$ contains an edge. Thus $\Gamma$ admits a leaf (a vertex connected to only one other vertex), say $v$. If the edge connected to $v$ points away from $v$, then $v$ is a source. Otherwise, removing $v$ and the edge attached to it, we obtain a tree with fewer vertices than $\Gamma$. If the tree obtained contains only one vertex (and hence no edges), that vertex is a sink of $\Gamma$. Otherwise, by induction the tree obtained admits a sink; it is clear that the same vertex is a sink for $\Gamma$ as well.

Let $X^{\prime}$ be a component of the JSJ decomposition of $X\left(\alpha^{\prime}\right)$ that corresponds to a source. By construction of $T(X), X^{\prime}$ is a direct descendant of $X\left(\alpha^{\prime}\right)$. We claim that $X(\alpha)$ is obtained from $X^{\prime}$ by filling (this allows for the possibility that $X^{\prime} \cong X(\alpha)$ and no boundary component is filled). To see this, let $T$ be a component of $\partial X^{\prime}$. We will denote by $Y$ the component of $X\left(\alpha^{\prime}\right)$ cut open along $T$ that is disjoint from $X^{\prime}$. As above, considering $T \subset X(\alpha)$, we see three cases:

(1) $T$ is parallel to a component of $\partial X(\alpha)$; equivalently, $Y\left(\left.\alpha\right|_{\partial Y}\right) \cong T^{2} \times[0,1]$. We remove $Y$ and the solid tori attached to it. The manifold obtained is not changed.

(2) $T$ bounds a solid torus outside $X^{\prime}$; equivalently, $Y\left(\left.\alpha\right|_{\partial Y}\right) \cong D^{2} \times S^{1}$. Again we remove $Y$ and the solid tori attached to it, and consider $X(\alpha)$ as the manifold obtained by filling $X^{\prime}$ along the induced slope (recall the definition of induced slope from item (7) in Section 2A). 

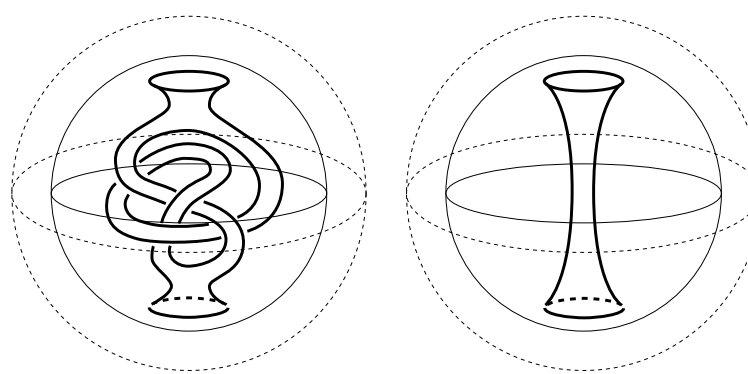

Figure 3: Unknotting in a ball

(3) $Y$ is a knot exterior contained in a ball $D$ : if $\partial Y$ is compressible then $Y \cong$ $D^{2} \times S^{1}$; this was treated in case (2), and we assume as we may that this does not happen. Thus $T$ is essential in $Y\left(\left.\alpha\right|_{\partial Y}\right)$, and since $X(\alpha)$ is atoroidal $T$ must compress in $X(\alpha)$ away from $Y$. Let $D$ be a compressing disk. It is now easy to see that replacing $Y$ with a solid torus such that the meridian of the solid torus intersects $\partial D$ exactly once does not change the ambient manifold. This can be seen as "unknotting" $T$ in $D$; see Figure 3 .

Repeating this process on all the components of $\partial X^{\prime}$, we see that $X(\alpha)$ is obtained from $X^{\prime}$ by attaching solid tori.

This completes the proof of Proposition 5.2.

So here is where we stand: if we fill to obtain a prime atoroidal manifold, Proposition 5.2 allows us to travel down $T(X)$ from a vertex at level $3 m+1$ or $3 m+2$. Assume, in addition, that $X(\alpha)$ is not hyperbolic. A manifold corresponding to a vertex labeled $3 m$ is Seifert fibered, sol or hyperbolic. The first two cases require direct analysis; in the final case we are guaranteed to have a minimally nonhyperbolic filling that allows us to keep going down $T(X)$.

The problem occurs when we want to study hyperbolic fillings. Obviously, one cannot expect every filling to admit a minimally nonhyperbolic partial filling; this is simply false. Somewhat surprisingly, we have the following proposition, which allows us to go down $T(X)$ in certain circumstances; recall that a multislope that does not admit a minimally nonhyperbolic partial filling is called totally hyperbolic:

Proposition 5.4 Let $X$ be a hyperbolic manifold and $\epsilon>0$. Let $\mathcal{A}$ be the set of multislopes $\alpha$ of $\partial X$ such that $X(\alpha)$ is hyperbolic and every geodesic in $X(\alpha)$ is longer than $\epsilon$.

Then there are only finitely many totally hyperbolic multislopes in $\mathcal{A}$. 
Remark 5.5 Since $\partial X$ may have arbitrarily many components, it is easy to construct examples of hyperbolic manifolds with infinitely many multislopes $\alpha$ such that $X(\alpha)$ is hyperbolic but does not admit a geodesic shorter than $\epsilon$, for a fixed $\epsilon>0$. This proposition allows us to study those: there is a finite set of totally hyperbolic multislopes, and every other multislope in $\mathcal{A}$ admits a minimally nonhyperbolic partial filling; these correspond to moving down $T(X)$.

Proof Denote the components of $\partial X$ by $T_{1}, \ldots, T_{n}$. We assume as we may that $\mathcal{A}$ is infinite. Let $\alpha^{j}=\left(\alpha_{1}^{j}, \ldots, \alpha_{n}^{j}\right)$ be an infinite set of multislopes in $\mathcal{A}$; we will prove the theorem by showing that some $\alpha^{j}$ admits a nonhyperbolic partial filling. The remainder of the proof is very similar to the proof of Proposition 4.2 and we only paraphrase it here.

After subsequencing, we may assume that for each $i$ one of the following holds:

(1) $\alpha_{i}^{j} \neq \alpha_{i}^{j^{\prime}}$ for $j \neq j^{\prime}$; we assume in addition that $\alpha_{i}^{j} \neq \infty$ for any $j$.

(2) $\alpha_{i}^{j}=\alpha_{i}^{j^{\prime}}$ for any $j, j^{\prime}$ (possibly $\alpha_{i}^{j}=\infty$ ).

After renumbering we may assume that the constant slopes are $\alpha_{i}^{j}$ for $i>k$ (for some $0 \leq k \leq n)$. Since $\mathcal{A}$ is infinite, $k \geq 1$. For $i>k$, we drop the superscript from $\alpha_{i}$.

Let $\hat{X}=X\left(\infty, \ldots, \infty, \alpha_{k+1}, \ldots, \alpha_{n}\right)$. Then $X\left(\alpha^{j}\right)=\hat{X}\left(\alpha_{1}^{j}, \ldots, \alpha_{k}^{j}\right)$. If $\hat{X}$ were hyperbolic, then by Thurston's Dehn surgery theorem for large enough $j$, we would obtain a hyperbolic manifold with $k$ geodesics of length less than $\epsilon$; as $k \geq 1$ this violates our assumption. Thus $\hat{X}$ is nonhyperbolic, and $\left(\infty, \ldots, \infty, \alpha_{k+1}, \ldots, \alpha_{n}\right) \subset_{\mathrm{pf}}$ $\left(\alpha_{1}^{j}, \ldots, \alpha_{k}^{j}, \alpha_{k+1}, \ldots, \alpha_{n}\right)$ is a nonhyperbolic partial filling (for any $j$ ). The proposition follows.

We end this section with the following lemma:

Lemma 5.6 Let $X$ be a JSJ manifold and $X_{0}$ a connected manifold that is obtained as the union of a strict subset of the components of the torus decomposition of $X$.

Then $\left|T\left(X_{0}\right)\right|<|T(X)|$.

Proof If $X_{0}$ is a component of the torus decomposition of $X$ then it corresponds to a direct descendant of the root of $T(X)$, and clearly $T\left(X_{0}\right) \varsubsetneqq T(X)$. The lemma follows in this case.

If $X_{0}$ is the union of more than one component of the torus decomposition of $X$, we embed $T\left(X_{0}\right)$ into $T(X)$ by placing the root of $T\left(X_{0}\right)$ at the root of $T(X)$ and using the edges the correspond to the components of the torus decomposition of $X$ 
that appear in $X_{0}$. By assumption this is a strict subset of the components of the torus decomposition of $X$, and hence not all the edges are used. After this embedding we can view $T\left(X_{0}\right)$ as a subtree of $T(X)$, and we see that $T\left(X_{0}\right) \varsubsetneqq T(X)$. The lemma follows.

\section{Cosmetic surgery on $T^{2} \times I$}

There are two types of theorems proved using $T(X)$. The first addresses the question "how much can a manifold get twisted when performing cosmetic surgery?" The second addresses the question "how many fillings can result in a manifold fulfilling such-and-such condition?" In the next three sections we prove theorems of the first type. Recall that a cosmetic surgery on $L \subset M$ is a surgery with multislope $\alpha$ such that $L(\alpha) \cong M$. Below we consider cosmetic surgery on links in $T^{2} \times[0,1]$. Note that $T^{2} \times[0,1]$ gives a natural projection from $T^{2} \times\{1\}$ to $T^{2} \times\{0\}$; however, after cosmetic surgery, this identification may change. Hence the image of a specific slope in $T^{2} \times\{1\}$ may give an infinite set after cosmetic surgeries (and this does in fact happen). The theorem below controls this set, and more generally, the image of a bounded set:

Theorem 6.1 Let $B$ be a bounded set of slopes of $T^{2} \times\{1\}, L$ a link in $T^{2} \times[0,1]$, and $\mathcal{A}=\left\{\alpha \mid L(\alpha) \cong T^{2} \times[0,1]\right\}$. For $\alpha \in \mathcal{A}$, let $B_{\alpha}$ be the set of slopes of $T^{2} \times\{0\}$ that are obtained by projecting $B$ via the natural projection.

Then $\bigcup_{\alpha \in \mathcal{A}} B_{\alpha}$ is bounded.

Proof We will denote $T^{2} \times[0,1] \backslash N(L)$ by $X$ and $\partial N(L)$ by $\mathcal{T}$. Note that we may regard $\mathcal{A}$ as a set of multislopes on $\mathcal{T} \subset \partial X$.

The proof is an induction on $|T(X)|$.

Assume that $X$ is reducible Let $X_{1}$ be the factor of the prime decomposition of $X$ that contains $T^{2} \times\{0\}$ and $T^{2} \times\{1\}$ (note that both are contained in the same factor). Then any $\alpha \in \mathcal{A}$ induces $\left.\alpha\right|_{\partial X_{1}}$, and $X_{1}\left(\left.\alpha\right|_{\partial X_{1}}\right) \cong T^{2} \times[0,1]$. Moreover, all the other factors of the prime decomposition of $X$ become $S^{3}$ after filling and therefore do not affect the identification of $T^{2} \times\{1\}$ with $T^{2} \times\{0\}$. Denote by $B_{\left.\alpha\right|_{\partial X_{1}}}$ the image of $B$ under the natural projection using the product structure of $X_{1}\left(\left.\alpha\right|_{\partial X_{1}}\right) \cong T^{2} \times[0,1]$. Thus $B_{\alpha}=B_{\left.\alpha\right|_{\partial X_{1}}}$ for every $\alpha \in \mathcal{A}$; therefore $\bigcup_{\alpha \in \mathcal{A}} B_{\alpha}=\bigcup_{\alpha \in \mathcal{A}} B_{\left.\alpha\right|_{\partial X_{1}}}$. Since $\left|T\left(X_{1}\right)\right|<|T(X)|$, we see by induction that $B_{\left.\alpha\right|_{\partial X_{1}}}$ is bounded. The proposition follows in this case.

We assume from now on that $X$ is irreducible. 
Assume that $X$ is Seifert fibered Fix a Seifert fibration of $X$ and a multislope $\alpha \in \mathcal{A}$. If $\left.\alpha\right|_{T_{i}}$ is a fiber for some $T_{i} \subset \mathcal{T}$, then $X(\alpha)$ contains a sphere that separates $T^{2} \times\{0\}$ from $T^{2} \times\{1\}$, which is impossible since $X(\alpha) \cong T^{2} \times[0,1]$. Hence the fibration of $X$ extends to a fibration of $T^{2} \times[0,1]$. We conclude that $X$ is obtained from $T^{2} \times[0,1]$ by removing fibers.

We claim that up to diffeomorphism the only Seifert fibration of $T^{2} \times[0,1]$ is the product of an annulus with $S^{1}$. To see this, simply note that if the base orbifold of a Seifert fibered manifold has at least one orbifold point and at least two boundary components, or if it has positive genus, then the manifold admits a filling that is not diffeomorphic to a lens space, $S^{1} \times S^{2}$ or $S^{3}$. Hence the base orbifold in any fibration of $T^{2} \times[0,1]$ is an annulus with no orbifold points; this proves the claim.

Back to the case at hand, we see that $X$ is obtained from the product of an annulus with $S^{1}$ by removing a set of $n$ curves that has the form $\left\{p_{1}, \ldots, p_{n}\right\} \times S^{1}$, showing that $X$ is the product of an $n$-times punctured annulus with $S^{1}$. We will denote the components of $\partial X \backslash \partial X(\alpha)$ by $T_{1}, \ldots, T_{n}$. Since $X(\alpha)$ results in a Seifert fibered manifold with no exceptional fiber, the core of the solid torus attached to $T_{i}$ is not an exceptional fiber, and hence has the form $p_{i} / 1$ in the Seifert notation $(i=1, \ldots, n)$. Suppose $n>1$. Following Seifert's original work [20], by performing $k$ twists about an annulus connecting fibers with Seifert invariants $p_{1} / q_{1}$ and $p_{i} / q_{i}$, the invariants change as follows (for an arbitrary $k \in \mathbb{Z}$ ):

$$
\frac{p_{1}}{q_{1}} \mapsto \frac{p_{1}+k q_{1}}{q_{1}} \quad \text { and } \quad \frac{p_{i}}{q_{i}} \mapsto \frac{p_{i}-k q_{i}}{q_{i}} .
$$

As in our case $q_{i}=1$, by choosing $k=p_{i}$, we may assume that the filling of $T_{i}$ is of the form $0 / 1$, and ignore it (for $i>1$ ). Thus we have reduced the problem to the case $n=1$. Let $A \subset X$ be an embedded vertical annulus (that is, an annulus that is given as a union of Seifert fibers) connecting $T_{1}$ with a Seifert fiber on $T^{2} \times\{0\}$. We will denote the Seifert fiber on $T^{2} \times\{0\}$ by $\gamma$. Surgery about a fiber with coefficient $1 / q$ is equivalent to $q$ twists about $A$; we see that the effect of the cosmetic surgery is the same as $D_{\gamma}^{n}$, an $n^{\text {th }}$ power of a Dehn twist about $\gamma$, for some $n \in \mathbb{Z}$. Hence the image of $B$ after all possible cosmetic surgeries on $L$ is

$$
\left\{D_{\gamma}^{n}(\beta) \mid \beta \in B, n \in \mathbb{Z}\right\} .
$$

By Proposition 3.6(3), this set is bounded.

We assume from now on that $X$ is irreducible and not Seifert fibered.

Assume that $\boldsymbol{X}$ is a JSJ manifold We will denote the set of tori of the JSJ decomposition by $\mathcal{F}$. Let $\Gamma$ be the graph dual to the torus decomposition as defined 
in Definition 2.1. Since $T^{2} \times[0,1]$ is connected and admits no nonseparating tori, $\Gamma$ is a tree. Note that $\Gamma$ has two (not necessarily distinct) vertices, denoted $v_{0}$ and $v_{1}$, that correspond to the components of the torus decomposition of $X$ that contain $T^{2} \times\{0\}$ and $T^{2} \times\{1\}$. There are two cases to consider:

Case $1\left(v_{0} \neq v_{1}\right)$ Let $e$ be an edge on the shortest path connecting $v_{0}$ and $v_{1}$ and $F \in \mathcal{F}$ the torus corresponding to $e$. In any filling, $F$ separates $T^{2} \times\{0\}$ from $T^{2} \times\{1\}$. Thus for every $\alpha \in \mathcal{A}$, we have that $F \subset X(\alpha) \cong T^{2} \times[0,1]$ is isotopic to $T^{2} \times\left\{\frac{1}{2}\right\}$. Let $X_{0}$ and $X_{1}$ be the components of $X$ cut open along $F$ so that $T^{2} \times\{i\} \subset X_{i}$ ( $i=0,1)$. We see that the fillings induced by $\alpha \in \mathcal{A}$ fulfill

$$
X_{i}\left(\left.\alpha\right|_{\partial X_{i}}\right) \cong T^{2} \times[0,1] \text {. }
$$

For $i=0,1$, let

$$
\mathcal{A}_{i}=\left\{\left.\alpha\right|_{\mathcal{F} \cap \partial X_{i}} \mid \alpha \in \mathcal{A}\right\} .
$$

Then for any $\alpha \in \mathcal{A}$ we have $X(\alpha)=X_{0}\left(\alpha_{0}\right) \cup X_{1}\left(\alpha_{1}\right)$, where $\alpha_{i}=\left.\alpha\right|_{\mathcal{F} \cap \partial X_{i}}$. By Lemma 5.6, we have $\left|T\left(X_{0}\right)\right|,\left|T\left(X_{1}\right)\right|<|T(X)|$. Applying induction to $X_{1}$, we conclude that

$$
\bigcup_{\alpha_{1} \in \mathcal{A}_{1}} B_{\alpha_{1}}
$$

is a bounded set of slopes of $F$; we will denote it by $B^{F}$. Next we apply induction to $X_{0}$ and conclude that

$$
\bigcup_{\alpha_{0} \in \mathcal{A}_{0}} B_{\alpha_{0}}^{F}
$$

is bounded. It is clear from the discussion above that $\bigcup_{\alpha \in \mathcal{A}} B_{\alpha} \subset \bigcup_{\alpha_{0} \in \mathcal{A}_{0}} B_{\alpha_{0}}^{F}$; the theorem follows in Case 1.

Case $2\left(v_{0}=v_{1}\right)$ The assumption means that both $T^{2} \times\{0\}$ and $T^{2} \times\{1\}$ are contained in the same component of the torus decomposition of $X$, say $X_{0}$. We will denote the components of $\operatorname{cl}\left(X \backslash X_{0}\right)$ by $X_{1}, \ldots, X_{k}$. Since $T^{2} \times[0,1]$ does not admit a nonseparating torus, if $X_{i} \cap X_{0}$ consists of more than one component (for some $i$ ) then $\mathcal{A}=\varnothing$ and there is nothing to prove. We assume as we may that for every $i$, $X_{i} \cap X_{0}$ is a single torus which we will denote by $T_{i}^{\prime}$. Note that $T_{i}^{\prime}$ is a component of the JSJ decomposition of $X$ and under the assumptions of Case 2 it cannot be boundary parallel in $X(\alpha) \cong T^{2} \times[0,1]$. Hence every $\alpha \in \mathcal{A}$ induces a filling on every $X_{i}$ fulfilling exactly one of the following conditions:

(1) $X_{i}\left(\left.\alpha\right|_{\partial X_{i}}\right) \cong D^{2} \times S^{1}$.

(2) $X_{i}\left(\left.\alpha\right|_{\partial X_{i}}\right) \cong E\left(K_{i}\right)$, where $K_{i} \subset S^{3}$ is a nontrivial knot and $X_{i}\left(\left.\alpha\right|_{\partial X_{i}}\right) \subset D_{i}$ for some ball $D_{i} \subset X(\alpha)$. 
By Lemma 2.2 we may assume that the balls $D_{i} \subset X(\alpha)$ are disjointly embedded. Given $\alpha \in \mathcal{A}$ we construct a multislope $\alpha_{0}$ of $\mathcal{T}_{0}$ as follows:

(1) If $X_{i}\left(\left.\alpha\right|_{\partial X_{i}}\right) \cong D^{2} \times S^{1}$ then $\left.\alpha_{0}\right|_{T_{i}}$ is the meridian of the solid torus $X_{i}\left(\left.\alpha\right|_{\partial X_{i}}\right)$.

(2) If $X_{i}\left(\left.\alpha\right|_{\partial X_{i}}\right) \cong E\left(K_{i}\right)$ we pick a slope that intersects the meridian of $E\left(K_{i}\right)$ exactly once (recall Figure 3 ).

Then $X_{0}\left(\alpha_{0}\right) \cong X(\alpha)$ and by construction the product structures of $X_{0}\left(\alpha_{0}\right)$ and $X(\alpha)$ induce the same projection from the slopes of $T^{2} \times\{1\}$ to those of $T^{2} \times\{0\}$.

Let $\mathcal{A}_{0}$ be the set of all multislopes $\alpha_{0}$ of $X_{0}$ such that $X_{0}\left(\alpha_{0}\right) \cong T^{2} \times[0,1]$. For each $\alpha_{0} \in \mathcal{A}_{0}$ we will denote the image of $B$ under the projection induced by the product structure by $B_{\alpha_{0}}$. If $\alpha_{0}$ was constructed as above for some $\alpha \in \mathcal{A}$, then $B_{\alpha}=B_{\alpha_{0}}$. Thus we see that

$$
\bigcup_{\alpha \in \mathcal{A}} B_{\alpha} \subset \bigcup_{\alpha_{0} \in \mathcal{A}_{0}} B_{\alpha_{0}}
$$

By Lemma 5.6, we have that $\left|T\left(X_{0}\right)\right|<|T(X)|$; hence by induction $\bigcup_{\alpha_{0} \in \mathcal{A}_{0}} B_{\alpha_{0}}$ is bounded. The theorem follows in Case 2 .

We assume from now on the $X$ is irreducible, not Seifert fibered, and not a JSJ manifold.

Assume that $X$ is sol Since $T^{2} \times\{0\} \subset \partial X$, we see that $\partial X \neq \varnothing$; hence $X$ cannot be sol.

We assume from now on that $X$ is irreducible, not Seifert fibered, not a JSJ manifold, and not sol. This leaves the following final case:

Assume that $\boldsymbol{X}$ is hyperbolic As $T^{2} \times[0,1]$ is nonhyperbolic, any multislope $\alpha$ with $X(\alpha) \cong T^{2} \times[0,1]$ admits a minimally nonhyperbolic partial filling. By Proposition 4.2 $X$ admits only finitely many minimally nonhyperbolic multislopes, say denoted by $\alpha_{1}, \ldots, \alpha_{k}$. For $1 \leq i \leq k$, we will denote $X\left(\alpha_{i}\right)$ by $X_{i}$. Let

$$
\mathcal{A}_{i}=\left\{\left.\alpha\right|_{\partial X_{i}} \mid \alpha \in \mathcal{A}, \alpha_{i} \subset_{\mathrm{pf}} \alpha\right\},
$$

that is, $\mathcal{A}_{i}$ consists of the multislopes induced on $X_{i}$ by multislopes $\mathcal{A}$ that admit $\alpha_{i}$ as a partial filling. For $\alpha_{i} \in \mathcal{A}_{i}$, we will denote by $B_{\alpha_{i}}$ the image of $B$ under the projection induced by the product structure of $X_{i}\left(\alpha_{i}\right)$. Since $X_{i}$ is a direct descendant of the root of $T(X)$, we have $\left|T\left(X_{i}\right)\right|<|T(X)|$. By induction $\bigcup_{\alpha_{i} \in \mathcal{A}_{i}} B_{\alpha_{i}}$ is bounded. Since every $\alpha \in \mathcal{A}$ admits a minimally nonhyperbolic partial filling, for every such $\alpha$ there exists $i$ such that

$$
X(\alpha)=X_{i}\left(\alpha_{i}\right)
$$


where $\alpha_{i}=\left.\alpha\right|_{\partial X_{i}} \in \mathcal{A}_{i}$. Hence

$$
\bigcup_{\alpha \in \mathcal{A}} B_{\alpha}=\bigcup_{i=1}^{k} \bigcup_{\alpha_{i} \in \mathcal{A}_{i}} B_{\alpha_{i}} .
$$

Since bounded sets are closed under finite union, $\bigcup_{\alpha \in \mathcal{A}} B_{\alpha}$ is bounded.

This concludes the proof of Theorem 6.1.

\section{Cosmetic surgery on solid tori}

Let $V$ be a solid torus and $L \subset V$ a link. In this section we address the following question: how many slopes on $\partial V$ become the boundary of a meridian disk after cosmetic surgery on $L$ ? Before stating the main theorem of this section, we consider some examples. If some component of $L$ is the core of $V$, then (trivially) the answer is every slope. Next, let $L^{\prime}=K_{1}^{\prime} \cup K_{2}^{\prime}$ be a two-component link, where $K_{1}^{\prime}$ is a knot that is not a torus knot and admits a nontrivial cosmetic surgery (see Gabai [4] and Berge [1]) and $K_{2}^{\prime}$ is a core. Assume further that $K_{2}^{\prime}$ was isotoped to be in a very "complicated" configuration with respect to $K_{1}^{\prime}$ (we allow $K_{2}^{\prime}$ to pass through $K_{1}^{\prime}$ during this isotopy, changing $L^{\prime}$ but not $K_{1}^{\prime}$ or $K_{2}^{\prime}$ ). Let $L=K_{1} \cup K_{2}$ be the image of $K_{1}^{\prime}$ and $K_{2}^{\prime}$ in the solid torus obtained by cosmetic surgery on $K_{1}^{\prime}$. Then, due to the isotopy discussed above, we expect that $K_{2}$ is not a core of the solid torus and certainly $K_{1}$ is not. However, $L$ admits cosmetic surgeries that realize every slope on the boundary of the solid torus as the boundary of the meridian disk. The trouble is that although $K_{2}$ is not a core of the solid torus it becomes a core after cosmetic surgery.

With this in mind we state the main result of this section; note that condition (2) of the theorem is equivalent to requiring that none of the cores of the attached solid tori is a core of $L(\alpha)$ :

Theorem 7.1 Let $V$ be a solid torus and $L \subset V$ a link. Consider the set $\mathcal{A}$ of multislopes of $L$ such that for any $\alpha \in \mathcal{A}$ the following two conditions hold:

(1) $L(\alpha) \cong D^{2} \times S^{1}$.

(2) $L\left(\alpha^{\prime}\right) \not T^{2} \times[0,1]$ for any $\alpha^{\prime} \subset_{\mathrm{pf}} \alpha$.

Then the set of slopes on $\partial V$ that bound a disk in $L(\alpha)$ (for some $\alpha \in \mathcal{A}$ ) is bounded.

Proof Let $X=V \backslash N(L)$ and denote the components of $\partial X$ by $T, T_{1}, \ldots, T_{n}$, with $T=\partial X$. We will denote $L(\alpha)$ by $X(\alpha)$; this is consistent with the notation of Section $2 \mathrm{~A}$ and should cause no confusion. We induct on $|T(X)|$. 
Assume $X$ is reducible Let $S \subset X$ be an essential separating sphere that realizes the decomposition $X=X^{\prime} \# X^{\prime \prime}$, so that $X^{\prime}$ is prime and $T \subset X^{\prime}$ (we are not assuming that $X^{\prime \prime}$ is prime). For any $\alpha \in \mathcal{A}, X(\alpha)$ is diffeomorphic to $D^{2} \times S^{1}$, which is prime. Therefore after filling one side of $S$ becomes a ball $D$ and the other becomes a solid torus whose boundary is $T$. We conclude that $\alpha$ induces $\left.\alpha\right|_{\partial X^{\prime}}$ and the following conditions hold:

(1) $X^{\prime}\left(\left.\alpha\right|_{\partial X^{\prime}}\right)$ is diffeomorphic to $D^{2} \times S^{1}$ and its meridian is the same slope of $T$ as the meridian of $X(\alpha)$.

(2) There does not exist $\left.\alpha^{\prime} \subset_{\mathrm{pf}} \alpha\right|_{\partial X^{\prime}}$ such that $X^{\prime}\left(\alpha^{\prime}\right) \cong T^{2} \times[0,1]$. Otherwise, there would be $\alpha^{\prime \prime} \subset_{\mathrm{pf}} \alpha$ such that $X\left(\alpha^{\prime \prime}\right) \cong T^{2} \times[0,1]$, contradicting our assumptions.

(3) $\left|T\left(X^{\prime}\right)\right|<|T(X)|$ : this is immediate from the construction of $T(X)$, since $X^{\prime}$ is a direct descendant of the root $X$.

Applying the inductive hypothesis, we conclude that the set of meridians of $X^{\prime}\left(\left.\alpha\right|_{\partial X^{\prime}}\right)$ for $\alpha \in \mathcal{A}$ is bounded; the theorem follows in this case.

We assume from now on that $X$ is irreducible.

Assume $X$ is Seifert fibered We fix a Seifert fibration on $X$ for the remainder of this case. Given a multislope $\alpha \in \mathcal{A}$ we will consider two cases, depending on whether or not there is a torus $T_{i}$ on which the slope defined by the Seifert fibration and the slope defined by $\alpha$ are the same.

Case $1\left(\left.\alpha\right|_{T_{i}}\right.$ is isotopic to a Seifert fiber for some $\left.i\right)$ We claim that the meridian of $X(\alpha)$ is the slope defined the Seifert fibration on $T$. To see this, consider the meridian disk of the solid torus attached to $T_{i}$, which we will denote by $D_{i}$, and a vertical annulus in $X$ connecting $T_{i}$ and $T$, which we will denote by $A_{i}$. After isotopy if necessary we may assume that $D_{i} \cap T_{i}=A_{i} \cap T_{i}$. Then $D_{i} \cup A_{i}$ is a disk properly embedded in $X(\alpha)$ whose boundary is isotopic to a Seifert fiber and hence essential in $T$; thus $D_{i} \cup A_{i}$ is a meridian disk of $X(\alpha)$. This proves the claim and shows that there is only one possible slope in Case 1.

Case $2\left(\left.\alpha\right|_{T_{i}}\right.$ is not isotopic to a fiber for any $\left.i\right)$ Under this assumption the fibration of $X$ extends to a fibration of $X(\alpha)$. Since $X(\alpha) \cong D^{2} \times S^{1}$, its base orbifold is $D^{2}$ with at most one orbifold point. Since $X$ is obtained from $X(\alpha)$ by removing fibers, we conclude that $X$ admits a Seifert fibration whose base orbifold is a punctured disk with at most one orbifold point. If there is no orbifold point, then there is an index $i$ such that, for any $i^{\prime} \neq i,\left.\alpha\right|_{T_{i^{\prime}}}$ intersects the fiber exactly once (possibly $\left.\alpha\right|_{T_{i}}$ intersects the fiber once as well). Define $\alpha^{\prime} \subset_{\text {pf }} \alpha$ to be the partial filling obtained by filling all the components but $T_{i}$, that is, by setting $\left.\alpha^{\prime}\right|_{T_{i}}=\infty$ and $\left.\alpha^{\prime}\right|_{T_{i^{\prime}}}=\left.\alpha\right|_{T_{i^{\prime}}}$ for 
all $i^{\prime} \neq i$. Then $X\left(\alpha^{\prime}\right)$ is a Seifert fiber space over an annulus with no exceptional fibers and hence $X\left(\alpha^{\prime}\right) \cong T^{2} \times[0,1]$, contradicting condition (2) of the theorem. We assume as we may that the base orbifold of $X$ has exactly one orbifold point. Denote the multiplicity of the critical fiber by $p \geq 2$. Then the meridian of $X(\alpha)$ intersects a fiber on $\partial X$ exactly $p$ times; this gives a bounded set by Proposition 3.6(4).

We assume from now on that $X$ is irreducible and not Seifert fibered.

Assume $\boldsymbol{X}$ is a JSJ manifold Let $X_{0}$ be the component of the JSJ decomposition of $X$ that contains $T$ and denote the components of $\partial X_{0} \backslash T$ by $F_{j}, j=1, \ldots, m$. We will denote the closures of the components of $X \backslash X_{0}$ by $X_{j}$. To avoid the situation where $X_{j}=\varnothing$, if $F_{j} \subset \partial X$ we push it slightly into the interior of $X_{0}$ so that $X_{j} \cong T^{2} \times[0,1]$ in that case. Since $D^{2} \times S^{1}$ admits no nonseparating tori, we assume as we may that $X_{j} \cap X_{j^{\prime}}=\varnothing$ for $j \neq j^{\prime}$. By reordering the indices of $X_{j}$ if necessary we may assume that $F_{j} \subset X_{j}$. Finally, given $\alpha \in \mathcal{A}$ and $1 \leq j \leq m$, we will denote the components of $X(\alpha)$ cut open along $F_{j}$ by $X(\alpha)_{j}^{+}$and $X(\alpha)_{j}^{-}$, with $\partial X(\alpha)_{j}^{+}=T \cup F_{j}$ and $\partial X(\alpha)_{j}^{-}=F_{j}$. Consider the following subsets $\mathcal{A}_{j} \subset \mathcal{A}$ (for $j=0, \ldots, m)$ :

(1) $\mathcal{A}_{0}$ consists of all the multislopes $\alpha \in \mathcal{A}$ such that $X(\alpha)_{j}^{+} \not \not T^{2} \times[0,1]$ for any $j$.

(2) For $1 \leq j \leq m, \mathcal{A}_{j}$ consists of all the multislopes $\alpha \in \mathcal{A}$ such that $X(\alpha)_{j}^{+} \cong$ $T^{2} \times[0,1]$.

It is immediate from the definitions that

$$
\mathcal{A}=\bigcup_{j=0}^{m} \mathcal{A}_{j} .
$$

We first consider multislopes $\alpha \in \mathcal{A}_{0}$. By definition of $\mathcal{A}_{0}$, no $F_{j}$ is boundary parallel in $X(\alpha) \cong D^{2} \times S^{1}$; thus every torus $F_{j}$ bounds a solid torus or a nontrivial knot exterior $E\left(K_{j}\right)$. For each $F_{j}$ that bounds a solid torus, let $\left.\hat{\alpha}\right|_{F_{j}}$ be the slope of $F_{j}$ defined by the meridian of that solid torus. For each torus $F_{j}$ that bounds a nontrivial knot exterior $E\left(K_{j}\right)$ we do the following: by Lemma 2.2 we may assume that $E\left(K_{j}\right) \subset D_{j}$ for disjointly embedded balls $D_{j}$. We replace every $E\left(K_{j}\right)$ with a solid torus (which we will denote by $\left.V_{j}\right)$ so that the meridian of $V_{j}$ intersects that meridian of $E\left(K_{j}\right)$ exactly once. This does not change the ambient manifold and, since the changes are contained in balls, the slope of $T$ that is the meridian of the solid torus $X(\alpha)$ is not changed. Let $\left.\hat{\alpha}\right|_{F_{j}}$ be the slope of $F_{j}$ defined by the meridian of $V_{j}$. Thus we have defined a slope $\left.\hat{\alpha}\right|_{F_{j}}$ for every $1 \leq j \leq m$; together they induce a multislope of $\left\{F_{j}\right\}_{j=1}^{m}$ which we will denote by $\hat{\alpha}$. We claim that $\hat{\alpha}$ fulfills the following two conditions: 
(1) $X_{0}(\widehat{\alpha}) \cong D^{2} \times S^{1}$ : this is immediate from the construction.

(2) There is no partial filling $\hat{\alpha}^{\prime} \subset_{\mathrm{pf}} \hat{\alpha}$ such that $X_{0}\left(\hat{\alpha}^{\prime}\right) \not T^{2} \times[0,1]$ : assume, for a contradiction, that such a partial filling $\hat{\alpha}^{\prime}$ exists. Since $T^{2} \times[0,1]$ has two boundary components, $\hat{\alpha}^{\prime}$ is obtained from $\hat{\alpha}$ by setting the value of $\left.\hat{\alpha}\right|_{F_{i}}$ to $\infty$ on exactly one torus $F_{j}$. By the defining condition for $\mathcal{A}_{0}$ this is impossible for values of $j$ for which $X(\alpha)_{j}^{-} \cong D^{2} \times S^{1}$, and for other values of $j$ this is impossible because $F_{j}$ is contained in the ball $D_{j} \subset X(\alpha)$.

Thus $\hat{\alpha}$ satisfies the assumptions of Theorem 7.1. By construction $X_{0}$ corresponds to a direct descendant of the root of $T(X)$, and therefore $\left|T\left(X_{0}\right)\right|<|T(X)|$. By induction, the set of meridians of the solid tori $X_{0}(\hat{\alpha})$ (as $\hat{\alpha}$ varies over all possible multislopes that correspond to the multislope $\alpha \in \mathcal{A}_{0}$ ) form a bounded set of slopes of $T$, which we will denote by $B_{0}$. By construction, the set of meridians of $X(\alpha)$ for $\alpha \in \mathcal{A}_{0}$ is $B_{0}$.

Next fix $1 \leq j \leq m$ and consider $\alpha \in \mathcal{A}_{j}$. By the defining condition for $\mathcal{A}_{j}$, $X(\alpha)_{j}^{+} \cong T^{2} \times[0,1]$. Hence $X(\alpha)_{j}^{-} \cong X(\alpha) \cong D^{2} \times S^{1}$. Note that $X(\alpha)_{j}^{-}$is obtained by filling a component of $X$ cut open along $F_{j}$ denoted above by $X_{j}$; the induced filling is given by $\left.\alpha\right|_{\partial X_{j}}$. We claim that the following conditions hold:

(1) $X_{j}\left(\left.\alpha\right|_{\partial X_{j}}\right) \cong D^{2} \times S^{1}:$ this is immediate from the construction.

(2) There is no partial filling $\left.\left.\alpha\right|_{\partial X_{j}} ^{\prime} \subset_{\mathrm{pf}} \alpha\right|_{\partial X_{j}}$ with $X_{j}\left(\left.\alpha\right|_{\partial X_{j}} ^{\prime}\right) \cong T^{2} \times[0,1]$ : otherwise, there would be a corresponding partial filling $\alpha^{\prime} \subset_{\mathrm{pf}} \alpha$ such that

$$
X\left(\alpha^{\prime}\right) \cong X(\alpha)_{j}^{+} \cup_{F_{j}} X_{j}\left(\left.\alpha\right|_{\partial X_{j}} ^{\prime}\right) \cong T^{2} \times[0,1]
$$

contradicting the assumptions of the theorem.

$$
\left|T\left(X_{j}\right)\right|<|T(X)| \text { : this follows from Lemma 5.6. }
$$

By induction, the set of slopes of meridians of $X_{j}\left(\left.\alpha\right|_{\partial X_{j}}\right)$ is bounded; we will denote it by $B_{j}^{\prime}$. By Theorem 6.1 the set of slopes of $T$ obtained by projecting $B_{j}^{\prime}$ after all possible cosmetic surgeries on $L \cap X(\alpha)_{j}^{+}$is bounded; we will denote it by $B_{j}$. Clearly, the set of meridians on $X(\alpha)$ (for $\alpha \in \mathcal{A}_{j}$ ) is contained in $B_{j}$.

We have obtained $m+1$ bounded sets, namely $B_{0}, \ldots, B_{m}$, such that the meridians of $X(\alpha)$ (for $\alpha \in \mathcal{A}$ ) are contained in $\bigcup_{j=0}^{m} B_{j}$. The theorem follows in this case.

We assume from now on that $X$ is irreducible, not Seifert fibered, and not a JSJ manifold.

Assume that $X$ is sol Since $T \subset \partial X$, we see that $\partial X \neq \varnothing$; hence $X$ cannot be sol.

We assume from now on that $X$ is irreducible, not Seifert fibered, not a JSJ manifold, and not sol. This leaves the following final case: 
Assume $\boldsymbol{X}$ is hyperbolic By Proposition $4.2 X$ admits only finitely many minimally nonhyperbolic fillings, which we will denote by $\alpha_{1}, \ldots, \alpha_{k}$. Fix $1 \leq j \leq k$. If there is no $\alpha \in \mathcal{A}$ for which $\alpha_{j} \subset_{\text {pf }} \alpha$, we set $B_{j}=\varnothing$; otherwise, any $\alpha \in \mathcal{A}$ for which $\alpha_{j} \subset_{\text {pf }} \alpha$ induces the multislope $\left.\alpha\right|_{\partial X\left(\alpha_{j}\right)}$ on $\partial X\left(\alpha_{j}\right)$. We claim that $X\left(\alpha_{j}\right)$ and $\left.\alpha\right|_{\partial X\left(\alpha_{j}\right)}$ fulfill the following four conditions:

(1) $X\left(\alpha_{j}\right)\left(\left.\alpha\right|_{\partial X\left(\alpha_{j}\right)}\right) \cong D^{2} \times S^{1}$ : this is immediate, as $X\left(\alpha_{j}\right)\left(\left.\alpha\right|_{\partial X\left(\alpha_{j}\right)}\right)=X(\alpha)$ (we emphasize that this is equality, not up to diffeomorphism).

(2) $\partial X\left(\alpha_{j}\right)\left(\left.\alpha\right|_{\partial X\left(\alpha_{j}\right)}\right)=T$ : this is immediate, as above.

(3) $\left.\alpha\right|_{\partial X\left(\alpha_{j}\right)}$ does not admit a partial filling $\left.\alpha^{\prime} \subset_{\mathrm{pf}} \alpha\right|_{\partial X\left(\alpha_{j}\right)}$ such that $X\left(\alpha_{j}\right)\left(\alpha^{\prime}\right) \cong$ $T^{2} \times[0,1]$ : otherwise the corresponding partial filling of $\alpha$ would yield $T^{2} \times[0,1]$, violating the second assumption of the theorem.

(4) The meridians of the solid tori $X\left(\alpha_{j}\right)\left(\left.\alpha\right|_{\partial X\left(\alpha_{j}\right)}\right)$ and $X(\alpha)$ define the same slope of $T$ : this is immediate, as emphasized in (1) above.

We will denote by $\mathcal{A}_{j} \subset \mathcal{A}$ the set

$$
\mathcal{A}_{j}=\left\{\left.\alpha\right|_{X\left(\alpha_{j}\right)} \mid \alpha \in \mathcal{A}, \alpha_{j} \subset_{\mathrm{pf}} \alpha\right\} .
$$

By points (1)-(3) above, $X\left(\alpha_{j}\right)$ and $\mathcal{A}_{j}$ fulfill the assumptions of the theorem. By construction $T\left(X\left(\alpha_{j}\right)\right)$ corresponds to a direct descendant of the root of $T(X)$; therefore $\left|T\left(X\left(\alpha_{j}\right)\right)\right|<|T(X)|$. By induction, the meridians of

$$
\left\{X\left(\alpha_{j}\right)\left(\left.\alpha\right|_{\partial X\left(\alpha_{j}\right)}\right) \mid \alpha \in \mathcal{A}_{j}\right\}
$$

form a bounded set of slopes of $T$, which we will denote by $B_{j}$. Since $D^{2} \times S^{1}$ is not hyperbolic, for every $\alpha \in \mathcal{A}$ there is $1 \leq j \leq k$ such that $\alpha_{j} \subset_{\mathrm{pf}} \alpha$; by point (4) above the meridian of $X(\alpha)$ is in $B_{j}$. We see that the meridians of $\{X(\alpha) \mid \alpha \in \mathcal{A}\}$ are

$$
\bigcup_{j=1}^{k} B_{j} .
$$

The theorem follows, as the finite union of bounded sets is bounded.

Next we prove a proposition about fillings that yield $D^{2} \times S^{1}$; it will be used in the proof of the ultimate claim in the paper, Proposition 14.6.

Proposition 7.2 Let $X$ be a compact orientable connected manifold such that $\partial X$ consists of tori. Denote the components of $\partial X$ by $T, T_{1}, \ldots, T_{n}$. Fix $\mathcal{T}$ a nonempty subset of $\left\{T_{1}, \ldots, T_{n}\right\}$. For a multislope $\alpha$ on $T_{1}, \ldots, T_{n}$, we will denote the link formed by the cores of the solid tori attached to $\mathcal{T}$ by $\mathcal{L}$. Let $\mathcal{A}$ be a set of multislopes of $\partial X$ such that every $\alpha \in \mathcal{A}$ satisfies the following conditions: 
(1) $X(\alpha) \cong D^{2} \times S^{1}$.

(2) $X(\alpha) \backslash \operatorname{int} N(\mathcal{L})$ is irreducible.

(3) No component of $\mathcal{L}$ is a core of $X(\alpha)$.

(4) $\left.\alpha\right|_{T}=\infty$.

Then for each $T_{i} \in \mathcal{T}$, there exists a bounded set $B_{T_{i}}$ of the slopes of $T_{i}$ such that for any $\alpha \in \mathcal{A}$ there exists $T_{i} \in \mathcal{T}$ with

$$
\left.\alpha\right|_{T_{i}} \in B_{T_{i}}
$$

Remark 7.3 For clarification we add a couple of comments. First note that conditions (1) and (4) imply that the boundary components filled are exactly $T_{1}, \ldots, T_{n}$. The link $\mathcal{L}$ has exactly $|\mathcal{T}|$ components, and it is certainly possible that $|\mathcal{T}|<n$. Whenever $|\mathcal{T}|<n$, the manifold $X(\alpha) \backslash$ int $M(\mathcal{L})$ is not homeomorphic to $X$ - they have different numbers of boundary components.

Proof We will induct on $|T(X)|$. Parts of the proof are similar to the proof of Theorem 7.1 and we will only sketch them here.

Assume that $X$ is hyperbolic Since $X(\alpha) \cong D^{2} \times S^{1}$ is not hyperbolic, any $\alpha \in \mathcal{A}$ factors through a minimally nonhyperbolic filling. Let $\alpha^{\prime} \subset_{\mathrm{pf}} \alpha$ be a minimally nonhyperbolic filling. If $\left.\alpha^{\prime}\right|_{T_{i}} \neq \infty$ for some $T_{i} \in \mathcal{T}$, we add $\left.\alpha^{\prime}\right|_{T_{i}}$ to $B_{T_{i}}$. We assume as we may that $\left.\alpha^{\prime}\right|_{T_{i}}=\infty$ for all $T_{i} \in \mathcal{T}$. Then $\mathcal{T} \subset \partial X\left(\alpha^{\prime}\right)$. We see that the filling $\left.\alpha\right|_{\partial X\left(\alpha^{\prime}\right)}$ induced by $\alpha$ on $X\left(\alpha^{\prime}\right)$ fulfills conditions (1)-(4) of the proposition; since $\left|T\left(X\left(\alpha^{\prime}\right)\right)\right|<|T(X)|$, the proposition follows from the induction hypothesis.

We assume from now on that $X$ is not hyperbolic.

Assume that $X$ is reducible Let $S$ be a sphere embedded in $X$ that realizes the decomposition $X^{\prime} \# X^{\prime \prime}$, where $X^{\prime}$ is prime and $T \subset X^{\prime}$ (we are not assuming that $X^{\prime \prime}$ is prime). By condition (2) of the proposition, $\mathcal{T} \subset \partial X^{\prime}$. Let $\mathcal{A}^{\prime}$ be the restrictions defined by

$$
\mathcal{A}^{\prime}=\left\{\left.\alpha\right|_{\partial X^{\prime}} \mid \alpha \in \mathcal{A}\right\} .
$$

It is easy to see that conditions (1)-(4) of the proposition hold, and since $\left|T\left(X^{\prime}\right)\right|<$ $|T(X)|$, the proposition follows from the induction hypothesis.

We assume from now on that $X$ is irreducible and not hyperbolic.

Assume that $\boldsymbol{X}$ is JSJ We first fix the notation that will be used in this case. Let $X_{0}$ be the component of the torus decomposition of $X$ that contains $T$. We will denote the components of $\partial X_{0}$ by $T, F_{1}, \ldots, F_{k}$ and the components of $\operatorname{cl}\left(X \backslash X_{0}\right)$ by 
$X_{1}, \ldots, X_{k}$, numbered so that $F_{j} \subset \partial X_{j}$. To avoid the situation $X_{j}=\varnothing$, if $F_{j} \subset \partial X$ we push it slightly into the interior so that $X_{j} \cong T^{2} \times[0,1]$ in this case. Since $D^{2} \times S^{1}$ contains no nonseparating tori, we assume as we may that $X_{j} \cap X_{j^{\prime}}=\varnothing$ for $j \neq j^{\prime}$. We will denote by $\mathcal{F} \subset\left\{F_{1}, \ldots, F_{k}\right\}$ the components $F_{j}$ that bound $X_{j}$ for which $\mathcal{T} \cap \partial X_{j} \neq \varnothing$. Given $\alpha \in \mathcal{A}$, we will denote $\left.\alpha\right|_{\partial X_{j}}$ by $\alpha_{j}$. (Note that by definition of $\mathcal{F}$, $F_{j} \in \mathcal{F}$ if and only if $\mathcal{L} \cap X_{j}\left(\alpha_{j}\right) \neq \varnothing$.) Clearly, $\partial X_{j}\left(\alpha_{j}\right)=F_{j}$ and $X_{j}\left(\alpha_{j}\right)$ is either a solid torus or the exterior of a nontrivial knot, which we will denote by $E\left(K_{j}\right)$. Up to finite ambiguity, we fix $\mathcal{F}_{\text {st }}$ and $\mathcal{F}_{\mathrm{k}}$ so that $\mathcal{F}=\mathcal{F}_{\text {st }} \sqcup \mathcal{F}_{\mathrm{k}}$ and consider the multislopes $\alpha \in \mathcal{A}$ for which $X_{j}\left(\alpha_{j}\right) \cong D^{2} \times S^{1}$ whenever $F_{j} \in \mathcal{F}_{\text {st }}$ and $X_{j}\left(\alpha_{j}\right) \cong E\left(K_{j}\right)$ whenever $F_{j} \in \mathcal{F}_{\mathrm{k}}$. To avoid overly complicated notation we do not rename $\mathcal{A}$. There are two cases to consider:

Case 1 (some $X_{j}\left(\alpha_{j}\right)$ has no core) Let $\mathcal{A}_{1} \subset \mathcal{A}$ be defined by requiring that for some $F_{j} \in \mathcal{F}_{\text {st }}$, no component of $\mathcal{L} \cap X_{j}\left(\alpha_{j}\right)$ is a core of the solid torus $X_{j}\left(\alpha_{j}\right)$. The second assumption of the proposition implies that $\mathcal{L} \cap X_{j}\left(\alpha_{j}\right)$ is irreducible. By Lemma 5.6, $\left|T\left(X_{j}\right)\right|<|T(X)|$. Applying the inductive hypothesis to $X_{j}$ we see that for each $T \in \mathcal{T} \cap \partial X_{j}$, there is a bounded set $B_{T}$ such that $\left.\alpha\right|_{T} \in B_{T}$ for some such $T$. The proposition follows for $\mathcal{A}_{1}$.

Case 2 (every $X_{j}\left(\alpha_{j}\right)$ has core) Let $\mathcal{A}_{2}=\mathcal{A} \backslash \mathcal{A}_{1}$. Then for every $F_{j} \in \mathcal{F}_{\text {st }}$ and every $\alpha \in \mathcal{A}_{2}$, the core of the solid torus attached to one of the components of $\mathcal{T} \cap \partial X_{j}$ is a core of $X_{j}\left(\alpha_{j}\right)$; we will denote it by $L_{j}$ (there may be more than one such component; we pick one). By Lemma 2.2, for every $j$ for which $F_{j} \in \mathcal{F}_{\mathrm{k}}$, there exists an embedded ball $D_{j} \subset X_{0}\left(\alpha_{0}\right)$ such that $X_{j}\left(\alpha_{j}\right) \subset D_{j}$ and $D_{j} \cap D_{j^{\prime}}=\varnothing$ for $j \neq j^{\prime}$. Thus the second assumption of the proposition implies that $\mathcal{F}_{\text {st }} \neq \varnothing$. Any $\alpha \in \mathcal{A}_{2}$ induces a multislope on $\partial X_{0}=T, F_{1}, \ldots, F_{k}$, which we will denote by $\alpha_{0}$, that consists of the following slopes:

(1) the meridian of $X_{j}\left(\alpha_{j}\right)$ (on components $F_{j}$ that bound $X_{j}\left(\alpha_{j}\right) \cong D^{2} \times S^{1}$ ),

(2) a slope that intersects the meridian of $X_{j}\left(\alpha_{j}\right)$ exactly once (on components $F_{j}$ that bound $X_{j}\left(\alpha_{j}\right) \cong E\left(K_{j}\right)$, a nontrivial knot exterior),

(3) $\infty($ on $T$ ).

For $F_{j} \in \mathcal{F}_{\mathrm{k}}$, the core of the solid tori attached to $F_{j}$ is an unknot in $D_{j}$; thus the cores of the solid tori attached to $\bigcup_{F_{j} \in \mathcal{F}_{\mathrm{k}}} F_{j}$ form a (possibly empty) unlink, which we will denote by $\mathcal{U}$. The cores of the solid tori attached to $\bigcup_{F_{j} \in \mathcal{F}_{\text {st }}} F_{j}$ form a (nonempty) link which we will denote by $\mathcal{L}_{0}$.

We claim that $\mathcal{L}_{0}$ is irreducible in the complement of $\mathcal{U}$. Assume, for a contradiction, that $X(\alpha) \backslash$ int $N\left(\mathcal{L}_{0} \cup \mathcal{U}\right)$ is reducible and let $S$ be a reducing sphere. Since $S$ is disjoint from the cores of the solid tori attached to $F_{j}$ (for every $F_{j} \in \mathcal{F}$ ) we may 
isotope $S$ out of these solid tori. It is now easy to see that $S \subset X(\alpha)$ and $S$ is disjoint from $\mathcal{L}$. Since $S$ is a reducing sphere for $\mathcal{L}_{0} \cup \mathcal{U}$, there are components of $\mathcal{L}_{0} \cup \mathcal{U}$ in the ball bounded by $S$ in $X_{0}\left(\alpha_{0}\right)$. Clearly, there are components of $\mathcal{L}$ in the ball bounded by $S$ in $X(\alpha)$. Thus $S$ is a reducing sphere for $\mathcal{L}$, contradicting the second assumption of the proposition.

We assume as we may by Lemma 2.4 that the slopes on $\mathcal{U}$ were chosen so that $\mathcal{L}_{0}$ is irreducible. We claim that no component of $\mathcal{L}_{0}$ is the core of $X_{0}\left(\alpha_{0}\right)$. Assume for a contradiction that this is not the case and fix $F_{j} \in \mathcal{F}_{\text {st }}$ for which the core of the solid torus attached to $F_{j}$ is a core of $X_{0}\left(\alpha_{0}\right)$. Recall that $L_{j}$ is a core of a solid torus attached to a component of $\mathcal{T} \cap \partial X_{j}$, and is the core of $X_{j}\left(\alpha_{j}\right)$. Thus $L_{j}$ is a component of $\mathcal{L}$, and is the core of $X(\alpha)$; this is impossible as it violates the third assumption of the proposition.

Thus $X_{0}, \mathcal{L}_{0}$, and the multislopes induced by $\mathcal{A}_{2}$ satisfy the assumptions of the proposition. By Lemma 5.6, we have $\left|T\left(X_{0}\right)\right|<T(X) \mid$. Hence by induction, for every $F_{j} \in \mathcal{F}_{\text {st }}$, there is a bounded set of slopes of $F_{j}$, which we will denote by $B_{F_{j}}$, so that for every multislope $\alpha_{0}$ (induced by some $\alpha \in \mathcal{A}_{2}$ ), there is $F_{j} \in \mathcal{F}_{\text {st }}$ for which $\left.\alpha_{0}\right|_{F_{j}} \in B_{F_{j}}$. The slope $\left.\alpha\right|_{\partial N\left(L_{j}\right)}$ is the projection of $\left.\alpha_{0}\right|_{F_{j}}$ by the product structure on $X_{j}\left(\alpha_{j}\right) \backslash N\left(L_{j}\right) \cong T^{2} \times[0,1]$. By the $T^{2} \times[0,1]$ cosmetic surgery theorem (Theorem 6.1) the projections of $B_{F_{j}}$ under all possible fillings of $X_{j}$ that yield $T^{2} \times[0,1]$ is a bounded set of slopes of $\partial N\left(L_{j}\right)$, which we will denote by $B_{j}$. Thus, for every $\alpha \in \mathcal{A}_{2}$, there exists $F_{j} \in \mathcal{F}_{\text {st }}$, for which $\left.\alpha\right|_{\partial N\left(L_{j}\right)} \in B_{j}$, proving the proposition in Case 2.

We assume from now on that $X$ is irreducible, not hyperbolic, and not JSJ.

Assume that $\boldsymbol{X}$ is a Seifert fibered space Fix a Seifert fibration on $X$. We consider three cases, depending on the intersection of $\left.\alpha\right|_{T_{i}}$ with slopes defined by the Seifert fiber on $T_{i}$ :

(1) If (for some $T_{i} \in \mathcal{T}$ ) $\left.\alpha\right|_{T_{i}}$ is the fiber in the Seifert fibration then the intersection number of $\left.\alpha\right|_{T_{i}}$ with the fiber is zero.

(2) If (for some $T_{i^{\prime}} \notin \mathcal{T}$ ) $\left.\alpha\right|_{T_{i^{\prime}}}$ is the fiber in the Seifert fibration, then for every $T_{i} \in \mathcal{T}$ the disk obtained by gluing a vertical annulus connecting $T_{i^{\prime}}$ to $T_{i}$ with a meridian disk of the solid torus attached to $T_{i^{\prime}}$ is a compressing disk for $T_{i}$ and its boundary is a regular fiber. Since $X(\alpha) \cong D^{2} \times S^{1}$ contains no nonseparating spheres or lens space summands, $\left.\alpha\right|_{T_{i}}$ intersects the regular fiber exactly once.

(3) If $\alpha_{T_{i}}$ is not the fiber for any $1 \leq i \leq n$, then the fibration on $X$ extends to a fibration of $X(\alpha)$, which is a fibration over $D^{2}$ with at most one exceptional fiber. The exceptional fiber, if exists, is the core of $X(\alpha)$. Thus by assumption (3) 
of the proposition every component of $\mathcal{L}$ is a regular fiber, implying that for every $T_{i} \in \mathcal{T},\left.\alpha\right|_{T_{i}}$ intersects the fiber in the Seifert fibration of $X$ exactly once.

We conclude that for every $\alpha \in \mathcal{A}$ there exists $T_{i} \in \mathcal{T}$ such that $\left.\alpha\right|_{T_{i}}$ intersects the fiber at most once. The proposition follows from Proposition 3.6(4).

We assume from now on that $X$ is irreducible, not hyperbolic, not JSJ, and not Seifert fibered. This leaves the following case:

Assume that $X$ is sol Since $T \subset \partial X$, we see that $\partial X \neq \varnothing$; hence $X$ cannot be sol. This completes the proof of Proposition 7.2.

\section{Hyperbolic cosmetic surgery: slopes}

In this section we ask "how much can a hyperbolic manifold get twisted by performing cosmetic surgery?" (Recall that in this paper by hyperbolic manifold we mean a connected compact manifold whose interior admits a complete finite-volume hyperbolic metric.) Consider the following problem: let $M$ be a hyperbolic manifold such that $\partial M$ is a single torus, $B$ a bounded set of slopes of $\partial M$, and $L \subset M$ a link. If $\alpha$ is a multislope of cosmetic surgery (that is, $L(\alpha) \cong M$ ), then an identification of $L(\alpha)$ with $M$ induces a bijection on the slopes of $\partial M$. Our goal is to show that the union of the images of $B$ under all such bijections is bounded. The theorem below is stated in terms of fillings (with $X$ corresponding to $M \backslash N(L)$ ) and is slightly more general as it allows for more boundary components.

Theorem 8.1 Let $M$ be an orientable hyperbolic manifold, $T_{M}$ a component of $\partial M$, $X$ a compact orientable connected manifold such that $\partial X$ consists of tori that we will denote by $T, T_{1}, \ldots, T_{n}$, and $B$ a bounded set of slopes of $T$. Let $\mathcal{X}=\left\{\left(\alpha, f_{\alpha}\right)\right\}$ be a set of pairs such that every $\left(\alpha, f_{\alpha}\right) \in \mathcal{X}$ satisfies the following conditions:

(1) $\alpha$ is a multislope of $X$.

(2) $f_{\alpha}: X(\alpha) \rightarrow M$ is a diffeomorphism.

(3) $f_{\alpha}$ maps $T$ to $T_{M}$.

For every $x=\left(\alpha, f_{\alpha}\right) \in \mathcal{X}$, we will denote the image of $B$ under the bijection induced by $f_{\alpha}$ from the slopes of $T$ to those of $T_{M}$ by $B_{x}$.

Then $\bigcup_{x \in \mathcal{X}} B_{x}$ is a bounded set of slopes of $T_{M}$. 
Proof We induct on $|T(X)|$.

Assume that $X$ is Seifert fibered or sol Then $X$ admits no hyperbolic filling.

We assume from now on that $X$ is not Seifert fibered or sol.

Assume that $X$ is reducible Let $S \subset X$ be a decomposing sphere that realizes the decomposition $X=X^{\prime} \cup_{S} X^{\prime \prime}$, where here $X^{\prime}$ is a prime manifold containing $T$ (we do not assume that $X^{\prime \prime}$ is prime). Then for every $\left(\alpha, f_{\alpha}\right) \in \mathcal{X}$ we have

$$
X(\alpha)=X^{\prime}\left(\left.\alpha\right|_{\partial X^{\prime}}\right) \cup_{S} X^{\prime \prime}\left(\left.\alpha\right|_{\partial X^{\prime \prime}}\right) .
$$

Since $X(\alpha) \cong M$ is hyperbolic, $S \subset X(\alpha)$ bounds a ball. Condition (3) of the theorem implies that $\left.\alpha\right|_{T}=\infty$; hence $X^{\prime \prime}\left(\left.\alpha\right|_{\partial X^{\prime \prime}}\right)$ is a ball. Thus $f_{\alpha}$ induces a diffeomorphism that we will denote by

$$
f_{\left.\alpha\right|_{\partial X^{\prime}}}: X^{\prime}\left(\left.\alpha\right|_{\partial X^{\prime}}\right) \rightarrow M
$$

We will denote the set of pairs $\left\{\left(\left.\alpha\right|_{\partial X^{\prime}}, f_{\left.\alpha\right|_{\partial X^{\prime}}}\right)\right\}$ induced by pairs $\left(\alpha, f_{\alpha}\right) \in \mathcal{X}$ by $\mathcal{X}^{\prime}$. For $\left(\left.\alpha\right|_{\partial X^{\prime}}, f_{\left.\alpha\right|_{\partial X^{\prime}}}\right)=x^{\prime} \in \mathcal{X}^{\prime}$, we will denote by $B_{X^{\prime}}$ the image of $B$ under the bijection induced by $f_{\left.\alpha\right|_{\partial X^{\prime}}}$ from the slopes of $T$ to those of $T_{M}$. Since $X^{\prime}$ corresponds to a direct descendant of the root of $T(X)$, we have $\left|T\left(X^{\prime}\right)\right|<|T(X)|$. By induction

$$
\bigcup_{x^{\prime} \in \mathcal{X}^{\prime}} B_{x^{\prime}}
$$

is a bounded set of slopes of $T_{M}$. By construction, every $x \in \mathcal{X}$ is induces $x^{\prime} \in \mathcal{X}^{\prime}$ for which $\left.f_{\left.\alpha\right|_{\partial X^{\prime}}}\right|_{T}=\left.f_{\alpha}\right|_{T}$. Thus $B_{x^{\prime}}=B_{x}$ and therefore

$$
\bigcup_{x \in \mathcal{X}} B_{x}=\bigcup_{x^{\prime} \in \mathcal{X}^{\prime}} B_{x^{\prime}}
$$

is a bounded set of slopes of $T_{M}$. The theorem follows in this case.

We assume from now on that $X$ is irreducible, not Seifert fibered, and not sol.

Assume that $\boldsymbol{X}$ is JSJ Let $\mathcal{A}$ be the set of multislopes in $\mathcal{X}$, that is, the following set of multislopes $\alpha$ :

$$
\mathcal{A}=\left\{\alpha \mid\left(\alpha, f_{\alpha}\right) \in \mathcal{X} \text { for some } f_{\alpha}\right\} .
$$

By Proposition 5.2, each $\alpha \in \mathcal{A}$ induces a filling on one of the components of the torus decomposition of $X$ that yields $M$. Up to finite ambiguity we fix one component of the torus decomposition of $X$, that we will denote as $X_{0}$, and consider only multislopes $\alpha$ that induce a filling on $X_{0}$. To avoid overcomplicated notation we do not rename $\mathcal{A}$. We will use the following notation: 
(1) The multislope induced by $\alpha$ on $\partial X_{0}$ will be denoted by $\alpha_{0}$.

(2) The closure of the component of $X \backslash X_{0}$ that contains $T$ will be denoted by $X_{1}$ (to avoid the situation $X_{1}=\varnothing$, if $T \subset \partial X_{0}$ we push it slightly into the interior so that $X_{1} \cong T^{2} \times[0,1]$ in this case).

(3) The torus $X_{0} \cap X_{1}$ will be denoted by $F$.

It follows from the construction in Proposition 5.2 that $f_{\alpha}(F)$ is a boundary parallel torus in $M$, which we will denote by $F_{M}$. We will denote the components of $M$ cut open along $F_{M}$ by $M_{0}$ and $M_{1}$, where $M_{1}$ is the component containing $T_{M}$. Each $\alpha \in \mathcal{A}$ induces a filling $X_{1}\left(\left.\alpha\right|_{\partial X_{1}}\right)$ whose result is $M_{1}$; since $F_{M}$ is a boundary parallel torus, $M_{1} \cong T^{2} \times[0,1]$. For each $\alpha \in \mathcal{A}$, the identification $X_{1}\left(\left.\alpha\right|_{\partial X_{1}}\right) \cong T^{2} \times[0,1]$ allows us to project $B$ to a set of slopes of $F$, which we will denote by $B_{F, \alpha}$. This is precisely the setup of Theorem 6.1, which tells us that the slopes

$$
\bigcup_{\alpha} B_{F, \alpha}
$$

form a bounded set of slopes of $F$, that we will denote by $B_{F}$.

By the construction in Proposition 5.2, $f_{\alpha}$ induces a diffeomorphism $f_{\alpha_{0}}: X_{0}\left(\alpha_{0}\right) \rightarrow M_{0}$ that maps $F$ to $F_{M}$. Since $X_{0}$ is a direct descendant of $X$, we have that $\left|T\left(X_{0}\right)\right|<$ $|T(X)|$. Therefore by induction the union of the images of $B_{F}$ under the bijections induced by $f_{\alpha_{0}}$ forms a bounded set of slopes of $F_{M}$, that we will denote by $B_{F_{M}}$. Since $M_{1} \cong T^{2} \times[0,1]$, the set $\bigcup_{x \in \mathcal{X}} B_{x}$ is naturally identified with $B_{F_{M}}$; hence $\bigcup_{x \in \mathcal{X}} B_{x}$ is bounded, completing the proof in this case.

We assume from now on that $X$ is irreducible and not Seifert fibered, sol, or JSJ.

Assume $\boldsymbol{X}$ is hyperbolic Let $\mathcal{X}^{1} \subset \mathcal{X}$ be all pairs $\left(\alpha, f_{\alpha}\right)$ for which $\alpha$ is totally hyperbolic. Fix $\alpha$ a totally hyperbolic filling and let $\left(\alpha, f_{j}\right)$ be all the elements of $\mathcal{X}^{1}$ that have $\alpha$ as their multislope, and different diffeomorphisms $f_{j}: X(\alpha) \rightarrow M$ (for $j \in J$ for some index set $J$ ). We will denote by $B_{j}$ the image of $B$ under the bijection induced by $f_{j}$ between the slopes of $T$ and those of $T_{M}$. After fixing an index in $J$, say $1 \in J$, we note that $f_{j} \circ f_{1}^{-1}: M \rightarrow M$ is a diffeomorphism that sends $T_{M}$ to itself and $f_{j}=\left(f_{j} \circ f_{1}^{-1}\right) \circ f_{1}$. Thus $B_{j}=\phi_{j}\left(B_{1}\right)$, where $\phi_{j}$ is a bijection induced on the slopes of $T$ by $f_{j} \circ f_{1}^{-1}$. By Lemma 2.5 , there exists a (possibly trivial) isometric involution $i$ on the set of slopes of $T_{M}$, so that for each $\phi_{j}$ we have that either $\phi_{j}\left(B_{1}\right)=B_{1}$ or $\phi_{j}\left(B_{1}\right)=i\left(B_{1}\right)$; since $i$ is an isometry, $i\left(B_{1}\right)$ is bounded. Hence

$$
\bigcup_{j \in J} B_{j} \subset B_{1} \cup i\left(B_{1}\right)
$$

is bounded. 
Let $\epsilon$ be the length of the shortest geodesic in $M$. By Proposition 5.4, $X$ admits only finitely many totally hyperbolic fillings not admitting a geodesic shorter than $\epsilon$, and hence

$$
\bigcup_{x \in \mathcal{X}^{1}} B_{x}
$$

is a bounded set of slopes.

Next we consider $\mathcal{X}^{2}=\mathcal{X} \backslash \mathcal{X}^{1}$. Since $\mathcal{X}^{1}$ consists of all totally hyperbolic multislopes in $\mathcal{X}$, every multislope in $\mathcal{X}^{2}$ admits a nonhyperbolic partial filling, and hence a minimally nonhyperbolic partial filling. By Proposition 4.2, $X$ admits only finitely many minimally nonhyperbolic fillings. We will denote by $\alpha_{1}, \ldots, \alpha_{k}$ the minimally nonhyperbolic fillings of $X$ for which $\left.\alpha_{j}\right|_{T}=\infty$. For $1 \leq j \leq k$, let $\mathcal{X}_{j}$ be the set of all pairs $\left(\alpha, f_{\alpha}\right)$ satisfying the following conditions:

(1) $\alpha$ is a multislope of $X\left(\alpha_{j}\right)$.

(2) $f_{\alpha}: X\left(\alpha_{j}\right)(\alpha) \rightarrow M$ is a diffeomorphism.

(3) $f_{\alpha}$ maps $T$ to $T_{M}$.

For $x_{j}=\left(\alpha, f_{\alpha}\right) \in \mathcal{X}_{j}$, we will denote by $B_{x_{j}}$ the image of $B$ under the bijection induced by $f_{\alpha}$ between the slopes of $T$ and those of $T_{M}$. Since $X\left(\alpha_{j}\right)$ corresponds to a direct descendant of the root of $T(X)$, we have $\left|T\left(X\left(\alpha_{j}\right)\right)\right|<|T(X)|$. Thus by induction

$$
\bigcup_{x_{j} \in \mathcal{X}_{j}} B_{x_{j}}
$$

is a bounded set of slopes of $T_{M}$.

By definition of $\mathcal{X}^{2}$, for every $x=\left(\alpha, f_{\alpha}\right) \in \mathcal{X}^{2}, \alpha$ is not totally hyperbolic. Hence $\alpha$ factors through some minimally nonhyperbolic filling $\alpha_{j}$ (for some $1 \leq j \leq k$ ), that is,

$$
X(\alpha)=X\left(\alpha_{j}\right)\left(\left.\alpha\right|_{\partial X\left(\alpha_{j}\right)}\right)
$$

We can view $f_{\alpha}: X(\alpha) \rightarrow M$ as a diffeomorphism $f_{\alpha}: X\left(\alpha_{j}\right)\left(\left.\alpha\right|_{\partial X\left(\alpha_{j}\right.}\right) \rightarrow M$; thus we obtain $\left(\left.\alpha\right|_{\partial X\left(\alpha_{j}\right)}, f_{\alpha}\right) \in \mathcal{X}_{j}$ for which $B_{\left(\left.\alpha\right|_{\partial X\left(\alpha_{j}\right)}, f_{\alpha}\right)}=B_{x}$. This shows that

$$
\bigcup_{x \in \mathcal{X}^{2}} B_{x} \subset \bigcup_{j=1}^{k}\left(\bigcup_{x_{j} \in \mathcal{X}_{j}} B_{x_{j}}\right) \text {. }
$$

Thus $\bigcup_{x \in \mathcal{X}^{2}} B_{x}$ is contained in a finite union of bounded sets, and hence is itself bounded.

The theorem follows in this final case.

This completes the proof of Theorem 8.1. 


\section{Hyperbolic cosmetic surgery: radius of injectivity}

Let $X$ be a hyperbolic manifold. A generic filling on $\mathcal{T} \subset \partial X$, with all the slopes very long, yields a hyperbolic manifold with at least $|\mathcal{T}|$ short geodesics. However, if $|\mathcal{T}|>1$, this requires excluding infinitely many multislopes. It is easy to construct examples where $X$ can be filled to give infinitely many manifolds that violate this rule; for example, every lens space is obtained by filling the Whitehead link exterior. As another example, given any hyperbolic manifold $M$, let $K \subset M \# T^{2} \times[0,1]$ be a simple knot. Then the exterior of $K$ is a hyperbolic manifold that admits infinitely many distinct multislopes $\alpha^{j}$ such that $X\left(\alpha^{j}\right) \cong M$ for every $j$ without the expected three short geodesics. In this section we show that although the set of multislopes yielding manifolds without a short geodesic may be infinite, only finitely many manifolds can be obtained.

Theorem 9.1 Let $X$ be a compact connected oriented manifold such that $\partial X$ consists of tori. Fix $\epsilon>0$. Then all but finitely many hyperbolic manifolds that are obtained by filling $X$ admit a geodesic of length less than $\epsilon$.

If in addition $X$ is hyperbolic, then there are only finitely many totally hyperbolic fillings $\alpha$ on $X$ such that $X(\alpha)$ does not admit a geodesic of length less than $\epsilon$.

Proof We induct on $|T(X)|$.

Assume that $X$ is Seifert fibered or sol Then no filling of $X$ yields a hyperbolic manifold.

We assume from now on that $X$ is not Seifert fibered or sol.

Assume that $\boldsymbol{X}$ is reducible Let $X_{1}, \ldots, X_{n}$ be the factors of the prime decomposition of $X$. Then in any filling of $X$ that gives a hyperbolic manifold (say $M$ ), exactly one $X_{i}$ fills to give $M$, and every other $X_{i^{\prime}}$ fills to $S^{3}$. Thus every hyperbolic manifold obtained by filling $X$ is obtained by filling $X_{i}$ for some $i$. Up to finite ambiguity we fix a factor $X_{i}$. Since $X_{i}$ corresponds to a direct descendant of $X$, we have $\left|T\left(X_{i}\right)\right|<|T(X)|$. By induction there are only finitely many hyperbolic manifolds obtained by filling $X_{i}$ that do not admit a geodesic of length less than $\epsilon$. The proposition follows in this case.

We assume from now on that $X$ is prime and not Seifert fibered or a sol manifold.

Assume that $\boldsymbol{X}$ is JSJ In order to apply Proposition 5.2 we start with a simple construction: let $Y$ be a hyperbolic manifold obtained by removing a simple knot from $X$ (such a knot exists by Myers [16, Corollary 6.3]) and let $\alpha^{\prime}$ be the multislope on $\partial Y$ 
given by the meridian on $\partial Y \backslash \partial X$ and $\infty$ on all other boundary components. Since $Y$ is hyperbolic, $Y\left(\alpha^{\prime}\right) \cong X$ is JSJ, and $\alpha^{\prime}$ corresponds to filling a single boundary component, it is clear that $\alpha^{\prime}$ is a minimally nonhyperbolic multislope. Applying Proposition 5.2 with $Y$ and $Y\left(\alpha^{\prime}\right)$ corresponding to $X$ and $X\left(\alpha^{\prime}\right)$ of that proposition, we conclude that any hyperbolic manifold obtained by filling $X \cong Y\left(\alpha^{\prime}\right)$ is obtained by filling some descendent of $X$ in $T(X)$. Let $X^{\prime}$ be a direct descendant of $X$. By construction, $\left|T\left(X^{\prime}\right)\right|<|T(X)|$. By induction, there only finitely many hyperbolic manifolds obtained by filling $X^{\prime}$ that do not admit a geodesic of length less than $\epsilon$. The proposition follows in this case, as $X$ has only finitely many direct descents.

We assume from now on that $X$ is irreducible and not JSJ, Seifert fibered, or sol. This leaves the following final case:

Assume that $\boldsymbol{X}$ is hyperbolic Let $\mathcal{A}$ be an infinite set of multislopes of $\partial X$ such that $X(\alpha)$ is hyperbolic and does not contain a geodesic shorter than $\epsilon$ for every $\alpha \in \mathcal{A}$. (Note that if no such set exists there is nothing to prove.) We will first establish the second conclusion of the theorem by showing that some multislope $\alpha \in \mathcal{A}$ is not totally hyperbolic. We will denote the components of $\partial X$ by $T_{1}, \ldots, T_{n}$. After subsequencing and reordering if necessary we assume as we may that for some $0 \leq k \leq n+1$ we have:

(1) For every $1 \leq i \leq k$ and every $j \neq j^{\prime}$ we have $\alpha_{i}^{j} \neq \alpha_{i}^{j^{\prime}}$ and $\alpha_{i}^{j} \neq \infty$.

(2) For every $k+1 \leq i \leq n$ and every $j, j^{\prime}$ we have $\alpha_{i}^{j}=\alpha_{i}^{j^{\prime}}$.

To avoid overly complicated notation we do not rename $\mathcal{A}$. Let $\alpha_{0}$ be the restriction $\left.\alpha\right|_{T_{k+1}, \ldots, T_{n}}$ for some $\alpha \in \mathcal{A}$ (by construction $\alpha_{0}$ is independent of choice), let $\hat{X}=$ $X\left(\alpha_{0}\right)$ (so $\partial \hat{X}=T_{1} \cup \cdots \cup T_{k}$ ), and let $\hat{\mathcal{A}}=\left\{\left.\alpha\right|_{\partial \hat{X}} \mid \alpha \in \mathcal{A}\right\}$. We claim that $\hat{X}$ is not hyperbolic; assume for a contradiction that it is. By truncating the cusps of $\hat{X}$ we obtain a Euclidean metric on every $T_{i}(1 \leq i \leq k)$. Since $\hat{\mathcal{A}}$ is infinite and the values $\left\{\left.\hat{\alpha}\right|_{T_{i}} \mid \hat{\alpha} \in \widehat{\mathcal{A}}\right\}$ are distinct, for any $l$ there is a multislope $\hat{\alpha} \in \hat{\mathcal{A}}$ such that $\left.\hat{\alpha}\right|_{T_{i}}$ is longer than $l$ for all $i$. By Thurston's Dehn surgery theorem, for large enough $l$, $\hat{X}(\hat{\alpha})$ is hyperbolic and the cores of the attached solid tori are geodesics of length less than $\epsilon$, contradicting our assumptions. Thus $\hat{X}$ is not hyperbolic. Since $\mathcal{A}$ is infinite, $k \geq 1$. By condition (1) above, for every $\alpha \in \mathcal{A}, \alpha_{0}$ is a strict partial filling of $\alpha$. This shows that $\alpha$ is not totally hyperbolic, establishing the second conclusion of the theorem.

We will denote the set of totally hyperbolic fillings in $\mathcal{A}$ by $\mathcal{A}_{-}$and $\mathcal{A} \backslash \mathcal{A}_{-}$by $\mathcal{A}_{+}$. Every $\alpha \in \mathcal{A}_{+}$admits a minimally nonhyperbolic partial filling, and the minimally nonhyperbolic fillings of $X$ correspond to the direct descendants of the root of $T(X)$; up to finite ambiguity we fix a direct descendant of the root of $X$ that we will denote 
by $X_{i}$. Then $\left|T\left(X_{i}\right)\right|<|T(X)|$. By induction there are only finitely many hyperbolic manifolds obtained by filling $X_{i}$ that do not admit a geodesic of length less than $\epsilon$. The theorem follows from this and finiteness of $\mathcal{A}_{-}$that was established above.

This completes the proof of Theorem 9.1.

\section{Cosmetic surgery on $S^{3}$}

We now turn to one of the more interesting applications of $T(X)$, concerning cosmetic surgery on $S^{3}$. Recall that a cosmetic surgery is $S^{3}$ is a surgery on a link $L \subset S^{3}$ with multislope $\alpha$ such that $L(\alpha) \cong S^{3}$. Note the following examples:

(1) Let $L=K_{1} \cup K_{2}$ be the Whitehead link. Then infinitely many slopes on $K_{1}$ can be completed to a cosmetic surgery: namely, $1 / m$ can be completed to the cosmetic surgery given by $(1 / m, 1 / 0)$, where here and in the examples below we are using the standard meridian-longitude. That is not a real problem: $\{1 / m\}$ is a bounded set.

(2) Worse is the Hopf link $H=K_{1} \cup K_{2}$. It is easy to see that $H(p / q, r / s) \cong S^{3}$ if and only if $p s-r q= \pm 1$. Thus every slope on $K_{1}$ can be completed to a cosmetic surgery.

(3) Kawauchi [13] constructed a two-component link $L=K_{1} \cup K_{2} \subset S^{3}$ that admits a nontrivial cosmetic surgery. By Teragaito [23, Theorem 1.1] we may assume that $L, K_{1}$ and $K_{2}$ are all hyperbolic (this was also announced by Kawauchi [12]). For a detailed discussion see the introduction to [23]. Let $L^{\prime} \subset S^{3}$ be the core of the attached solid tori after this surgery, and let $H^{\prime} \subset S^{3}$ be the Hopf link. By isotopy of $H^{\prime}$ (where we allow $H^{\prime}$ to intersect $L^{\prime}$ ) we place $H^{\prime}$ in a "very complicated" position relative to $L^{\prime}$. There is a surgery on $L^{\prime}$ which "undoes" the surgery, resulting in $S^{3}$. Denote the image of $H^{\prime}$ under this surgery by $H=K_{3} \cup K_{4}$. For any slope $\alpha_{3}$ there exist infinitely many slopes $\alpha_{4}$ such that $L \cup H\left(\alpha_{1}, \alpha_{2}, \alpha_{3}, \alpha_{4}\right) \cong S^{3}$. However, we expect that $H$ is no longer the Hopf link; in fact, it is quite likely that the components of $H$ are no longer unknotted, as the disks bounded by the components of $H^{\prime}$ are likely to be destroyed by the surgery on $L^{\prime}$, and new disks are unlikely to appear. We do not prove these claims, but in light of this discussion we expect the following to be true: there exists a four-component link in $S^{3}$ (such as $L \cup H=K_{1} \cup K_{2} \cup K_{3} \cup K_{4}$ above) that contains no Hopf sublink (perhaps even no unknotted components), yet every slope on $K_{3}$ can be completed to a cosmetic surgery. The moral is this: it is our aim to prove that not any slope can be completed to a cosmetic surgery, but one must beware of Hopf links, including those that are invisible in the original link but manifest themselves after surgery.

We are now ready to state: 
Theorem 10.1 Let $L \subset S^{3}$ be a link and denote its components by $K_{1}, \ldots, K_{n}$. Let $T_{1}$ be the component of $\partial E(L)$ that corresponds to $K_{1}$. Let $\mathcal{A}$ be a set of multislopes of $L$ such that every $\alpha \in \mathcal{A}$ fulfills the following two conditions:

(1) $L(\alpha) \cong S^{3}$.

(2) $L\left(\alpha^{\prime}\right) \not T^{2} \times[0,1]$ for any $\alpha^{\prime} \subset_{\mathrm{pf}} \alpha$ with $\left.\alpha^{\prime}\right|_{T_{1}}=\infty$.

Then the restrictions $\mathcal{A}_{1}=\left\{\left.\alpha\right|_{T_{1}} \mid \alpha \in \mathcal{A}\right\}$ form a bounded set.

Remarks 10.2 (1) There exists $\alpha^{\prime} \subset_{\mathrm{pf}} \alpha$ with $\left.\alpha^{\prime}\right|_{T_{1}}=\infty$ and $L\left(\alpha^{\prime}\right) \cong T^{2} \times[0,1]$ if and only if the cores of the solid tori attached to $\partial N\left(K_{1}\right)$ and $\partial N\left(K_{i}\right)$ form a Hopf link (for some $2 \leq i \leq n$ ). The cores of the solid tori attached along a multislope in $\mathcal{A}$ may, in fact, contain a Hopf sublink $H$; our assumption only requires that the core of the solid torus attached to $\partial N\left(K_{1}\right)$ is not a component of $H$.

(2) The second assumption in Theorem 10.1 cannot be avoided. If there exists $\alpha^{\prime} \subset_{\mathrm{pf}} \alpha$ with $\left.\alpha^{\prime}\right|_{T_{1}}=\infty$ and $L\left(\alpha^{\prime}\right) \cong T^{2} \times[0,1]$ then obviously any slope of $T_{1}$ can be completed to a cosmetic surgery.

Example 10.3 The following example shows that there is no explicit bound possible on the diameter of $\mathcal{A}_{1}$ in Theorem 10.1. Let $L=K_{1} \cup K_{2} \subset S^{3}$ be a two-component link such that $K_{1}$ is the unknot and $K_{2}$ is a torus knot on a torus parallel to $T_{1}=\partial N\left(K_{1}\right)$. Note that the exterior of $K_{1}$ is a solid torus, which we will denote by $V$, and $K_{2} \subset V$ is parallel to $\partial V=T_{1}$. Let $A$ be the annulus that $K_{2}$ cobounds with $T_{1}$. We will denote the slope of $T_{1}$ defined by $A \cap T_{1}$ by $\alpha$ and the slope of $T_{1}$ that corresponds to a curve that bounds a disk in $V$ by $\lambda$. Note that by choosing $K_{2}$ appropriately we can realize any slope of $T_{1}$ as $\alpha$, and in particular, $d_{\text {Farey }}(\lambda, \alpha)$ can be made arbitrarily large. We identify the slopes of $K_{2}$ with $\mathbb{Q} \cup\{1 / 0\}$ so that $1 / 0$ corresponds to the meridian in $S^{3}$ and $0 / 1$ is the slope defined by $A \cap \partial N\left(K_{2}\right)$. Then the slopes that intersect $A \cap \partial N\left(K_{2}\right)$ once are exactly those identified with $1 / n$. Surgery on $K_{2} \subset V$ with slope $1 / n$ yields a solid torus in which the slope $D_{\alpha}^{n}(\mu)$ bounds a disk (recall that $D_{\alpha}^{n}(\mu)$ is the slope obtained by Dehn twisting $\mu n$ times about $\alpha$ ). It is easy to see that the set of slopes of $T_{1}$ that corresponds to $\mathcal{A}_{1}$ (with the notation of Theorem 10.1) is

$$
\left\{\beta \mid d_{\text {Farey }}\left(\beta, D_{\alpha}^{n}(\mu)\right)=1 \text { for some } n \in \mathbb{Z}\right\} .
$$

On the other hand, for large enough $n$, we have $d_{\text {Farey }}\left(\mu, D_{\alpha}^{n}(\mu)\right)=2 d_{\text {Farey }}(\mu, \alpha)$. As $d_{\text {Farey }}(\mu, \alpha)$ can be arbitrarily large, we see that Theorem 10.1 cannot be improved by giving an explicit bound on the diameter of the set $\mathcal{A}_{1}$. If $K_{3}$ is a simple knot in $E(L)$, then $L^{\prime}=L \cup K_{3}$ provides a hyperbolic example with the same property as $L$. 
Proof of Theorem 10.1 We will denote $\operatorname{cl}\left(S^{3} \backslash N(L)\right)$ by $X$ and $\partial N\left(K_{i}\right)$ by $T_{i}$. Although the theorem was phrased in terms of surgery, we will prove the equivalent statement for fillings of $X$. We induct on $|T(X)|$.

Assume that $X$ is reducible Let $X=X^{\prime} \# X^{\prime \prime}$, where $X^{\prime}$ is the factor of the prime decomposition of $X$ that contains $T_{1}$. By renumbering the components of $\partial X$ if necessary we assume as we may that $\partial X^{\prime}=T_{1}, \ldots, T_{k}$, for some $1 \leq k \leq n$. For any multislope $\alpha \in \mathcal{A}$ we have

$$
X(\alpha) \cong X^{\prime}\left(\left.\alpha\right|_{\partial X^{\prime}}\right) \# X^{\prime \prime}\left(\left.\alpha\right|_{\partial X^{\prime \prime}}\right) .
$$

Thus $X^{\prime}\left(\left.\alpha\right|_{\partial X^{\prime}}\right) \cong S^{3} \cong X^{\prime \prime}\left(\left.\alpha\right|_{\partial X^{\prime \prime}}\right)$. If

$$
X^{\prime}\left(\infty, \alpha_{2}, \ldots, \alpha_{i-1}, \infty, \alpha_{i+1}, \ldots, \alpha_{k}\right) \cong T^{2} \times[0,1]
$$

for some $2 \leq i \leq k$, then

$$
\begin{aligned}
L\left(\infty, \alpha_{2}, \ldots, \alpha_{i-1}, \infty, \alpha_{i+1}\right. & \left., \ldots, \alpha_{n}\right) \\
& \cong X^{\prime}\left(\infty, \alpha_{2}, \ldots, \alpha_{i-1}, \infty, \alpha_{i+1}, \ldots, \alpha_{k}\right) \# X^{\prime \prime}\left(\left.\alpha\right|_{\partial X^{\prime \prime}}\right) \\
& \cong T^{2} \times[0,1] \# S^{3} \\
& \cong T^{2} \times[0,1] .
\end{aligned}
$$

This contradicts the second assumption of the theorem. Thus $X^{\prime}$ and $\mathcal{A}^{\prime}=\left\{\left.\alpha\right|_{\partial X^{\prime}} \mid \alpha \in \mathcal{A}\right\}$ fulfill the assumptions of the theorem. Since $X^{\prime}$ corresponds to a direct descendant of the root of $T(X)$, we have $\left|T\left(X^{\prime}\right)\right|<|T(X)|$. By induction, $\mathcal{A}_{1}^{\prime}=\left\{\left.\alpha^{\prime}\right|_{T_{1}} \mid \alpha^{\prime} \in \mathcal{A}^{\prime}\right\}$ is bounded. It is easy to see that $\mathcal{A}_{1}=\mathcal{A}_{1}^{\prime}$; the theorem follows in this case.

We assume from now on that $X$ is irreducible.

Assume that $\boldsymbol{X}$ is Seifert fibered or a sol manifold Since sol manifolds are closed we may ignore them. If $n=1$ then $L$ is a knot and the result is well known; for the rest of this case we assume as we may that $n \geq 2$. We fix a Seifert fibration on $X$. Then the fibers on $T_{1}$ define a slope which we denote by $\alpha_{1}^{f}$. For convenience we will denote $\left.\alpha\right|_{T_{i}}$ by $\alpha_{i}$. Define $\mathcal{A}_{f}, \mathcal{A}_{0}, \mathcal{A}_{1} \subset \mathcal{A}$ as follows:

(1) $\alpha \in \mathcal{A}_{f}$ if $\alpha_{1}=\alpha_{1}^{f}$.

(2) $\alpha \in \mathcal{A}_{0}$ if for some $2 \leq i \leq n, \alpha_{i}$ is the fiber on $T_{i}$.

(3) $\alpha \in \mathcal{A}_{1}$ if $\alpha \notin \mathcal{A}_{f} \cup \mathcal{A}_{0}$.

Clearly, $\mathcal{A}=\mathcal{A}_{f} \cup \mathcal{A}_{0} \cup \mathcal{A}_{1}$.

If $\alpha \in \mathcal{A}_{f}$ then $\alpha_{1} \in B_{f}$, where $B_{f}$ is the singleton defined by $B_{f}=\left\{\alpha_{1}^{f}\right\}$. Obviously, $B_{f}$ is the bounded set of slopes of $T_{1}$. 
For $\alpha \in \mathcal{A}_{0}$, let $D$ be the disk obtained by attaching a vertical annulus connecting $T_{i}$ and $T_{1}$ to the meridian disk of the solid torus attached to $T_{i}$ (where $i$ is as in the definition of $\left.\mathcal{A}_{0}\right)$. Thus we see that $D$ is a compressing disk for $T_{1}$ and the slope defined by $\partial D$ is $\alpha_{1}^{f}$. Since $X\left(\alpha_{1}, \ldots, \alpha_{n}\right) \cong S^{3}$, we have that $\Delta\left(\alpha_{1}, \alpha_{1}^{f}\right)=1$; thus $\alpha_{1} \in \Delta\left(\left\{\alpha_{1}^{f}\right\}, 1\right)$ (note that $\Delta$ is used in two different ways here; recall Notation 3.5). We will denote $\Delta\left(\left\{\alpha_{1}^{f}\right\}, 1\right)$ by $B_{0}$. By Proposition 3.6(4) we have that $B_{0}$ is a bounded set.

If $\alpha \in \mathcal{A}_{1}$ then the fibration of $X$ extends to a fibration of $X\left(\infty, \alpha_{2}, \ldots, \alpha_{n}\right)$, and the fibration of $X\left(\infty, \alpha_{2}, \ldots, \alpha_{n}\right)$ extends to a fibration of $X(\alpha) \cong S^{3}$. Therefore $X\left(\infty, \alpha_{2}, \ldots, \alpha_{n}\right)$ is a Seifert fibered space over $D^{2}$ with at most two exceptional fibers and the cores of the solid tori attached to $T_{1}, \ldots, T_{n}$ are fibers.

Assume first that $X\left(\infty, \alpha_{2}, \ldots, \alpha_{n}\right)$ is a Seifert fibered space over $D^{2}$ with exactly two exceptional fibers. Since $S^{3}$ does not admit a Seifert fibration with more than two exceptional fibers, we have that $\alpha_{1} \in B_{0}$ in this case.

Next assume that $X\left(\infty, \alpha_{2}, \ldots, \alpha_{n}\right)$ is a Seifert fibered space over $D^{2}$ with at most one exceptional fiber, and the exceptional fiber (if it exists) is the core of the solid torus attached to $T_{i}$; by renumbering $T_{2}, \ldots, T_{n}$ if necessary we may assume that $i=2$. Then $X\left(\infty, \infty, \alpha_{3}, \ldots, \alpha_{n}\right) \cong T^{2} \times[0,1]$, contradicting our assumption.

Thus we have reduced the proof to the case where $X\left(\infty, \alpha_{2}, \ldots, \alpha_{n}\right)$ is a Seifert fibered space over $D^{2}$ with exactly one exceptional fiber, and the exceptional fiber is not the core of a solid torus attached to $T_{i}(2 \leq i \leq n)$. Then the exceptional fiber is contained in $X$ and its multiplicity, which we will denote by $d$, does not depend on $\alpha \in \mathcal{A}_{1}$. Since $X\left(\infty, \alpha_{2}, \ldots, \alpha_{n}\right)$ is a Seifert fibered space over $D^{2}$ with exactly one exceptional fiber, $X\left(\infty, \alpha_{2}, \ldots, \alpha_{n}\right) \cong D^{2} \times S^{1}$; we will denote the slope defined by the boundary of its meridian disk by $\alpha^{\prime}$. Then $\Delta\left(\alpha_{1}^{f}, \alpha^{\prime}\right)=d$. Since $X(\alpha) \cong S^{3}$, we also have that $\Delta\left(\alpha^{\prime}, \alpha_{1}\right)=1$. Thus $\alpha_{1} \in B_{1}$, where $B_{1}$ is the set of slopes of $T_{1}$ defined by

$$
B_{1}=\left\{\alpha \mid \Delta\left(\alpha_{1}^{f}, \alpha^{\prime}\right)=d \text { and } \Delta\left(\alpha^{\prime}, \alpha\right)=1 \text { for some } \alpha^{\prime}\right\} \text {. }
$$

By applying Proposition 3.6(4) twice we see that $B_{1}$ is bounded.

Since $\mathcal{A}=\mathcal{A}_{f} \cup \mathcal{A}_{0} \cup \mathcal{A}_{1}$, for any $\alpha \in \mathcal{A}$ we have $\alpha_{1} \in B_{f} \cup B_{0} \cup B_{1}$. This completes the proof for Seifert fibered and sol manifolds.

We assume from now on that $X$ is irreducible, and not a Seifert fibered or sol manifold.

Assume that $\boldsymbol{X}$ is JSJ Let $X_{0}$ be the component of the torus decomposition of $X$ that contains $T_{1}$ and denote the remaining components of $\partial X_{0}$ by $\left\{F_{j}\right\}_{j=1}^{k}$; see Figure 4 . Given $\alpha \in \mathcal{A}$, we will denote the components of $X\left(\left.\alpha\right|_{T_{2}, \ldots, T_{n}}\right)$ cut open along $F_{j}$ as follows (see the right side of Figure 4): 

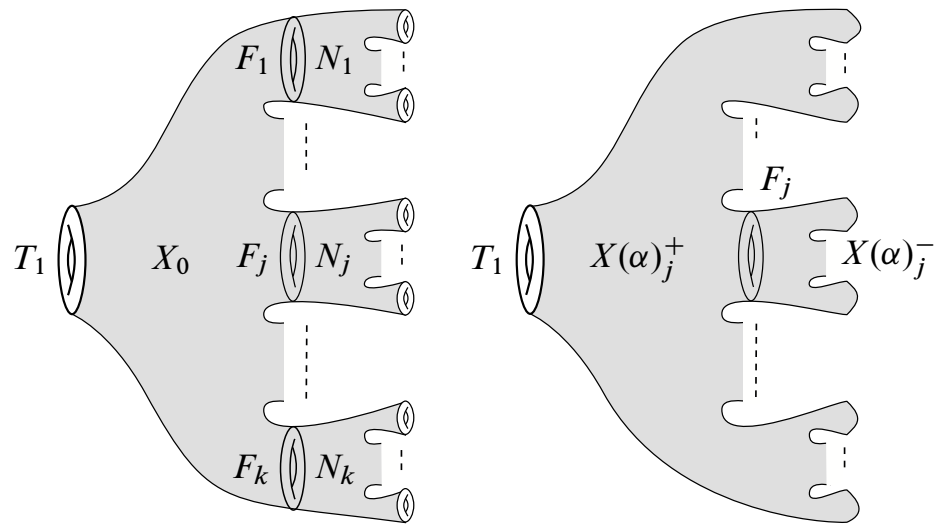

Figure 4: Notation used when $X$ is JSJ

(1) The component whose boundary is $T_{1} \cup F_{j}$ will be denoted by $X(\alpha)_{j}^{+}$.

(2) The component whose boundary is $F_{j}$ will be denoted by $X(\alpha)_{j}^{-}$.

Since $S^{3}$ admits no nonseparating torus, we assume as we may that $X(\alpha)_{j}^{-} \cap X(\alpha)_{j^{\prime}}^{-}=\varnothing$ for $j \neq j^{\prime}$.

There are two cases to consider:

Case 1 Let $\mathcal{A}_{1}$ be the multislopes $\alpha \in \mathcal{A}$ for which $X(\alpha)_{j}^{+} \not T^{2} \times[0,1]$ for all $j$. Then $\alpha \in \mathcal{A}_{1}$ induces a multislope on $\partial X_{0}$, which we will denote by $\alpha_{0}$, defined as follows:

(1) $\left.\alpha_{0}\right|_{T_{1}}=\left.\alpha\right|_{T_{1}}$.

(2) If $X(\alpha)_{j}^{-} \cong D^{2} \times S^{1}$ then $\left.\alpha_{0}\right|_{F_{j}}$ is the slope of the meridian of the solid torus $X(\alpha)_{j}^{-}$.

(3) If $X(\alpha)_{j}^{-} \neq D^{2} \times S^{1}$ then $X(\alpha)_{j}^{-} \cong E\left(K_{j}\right)$ for some nontrivial knot $K_{j} \subset S^{3}$. We take $\left.\alpha_{0}\right|_{F_{j}}$ to be any slope that intersects the meridian of $E\left(K_{j}\right)$ exactly once.

By Lemma 2.2, we may assume that the components $X(\alpha)_{j}^{-}$in Case (3) are contained in disjointly embedded balls; hence removing $X(\alpha)_{j}^{-}$and attaching a solid torus along $\left.\alpha_{0}\right|_{F_{j}}$ does not change $X(\alpha)$ or $X(\alpha)_{j^{\prime}}^{+}$(for $1 \leq j^{\prime} \leq k$ ); recall Figure 3 . Thus $X_{0}$ and the induced slopes $\left\{\alpha_{0} \mid \alpha \in \mathcal{A}_{1}\right\}$ satisfy the conditions of the theorem (condition (1) follows from the corresponding assumption for $X$, and condition (2) follows from the defining assumption of case one). By Lemma 5.6, we have $\left|T\left(X_{0}\right)\right|<|T(X)|$. By induction, $\left\{\left.\alpha_{0}\right|_{T_{1}} \mid \alpha \in \mathcal{A}_{1}\right\}$ is a bounded set which we will denote by $B_{1}$. By construction $\left.\alpha_{0}\right|_{T_{1}}=\left.\alpha\right|_{T_{1}}$.

Hence $\left\{\left.\alpha\right|_{T_{1}} \mid \alpha \in \mathcal{A}_{1}\right\}=B_{1}$ is bounded. 
Case 2 Fix $1 \leq j \leq k$. Let $\mathcal{A}_{2, j} \subset \mathcal{A}$ be the multislopes $\alpha \in \mathcal{A}$ for which $X(\alpha)_{j}^{+} \cong$ $T^{2} \times[0,1]$. The definitions immediately imply that the following two conditions hold:

(1) $X(\alpha)_{j}^{+}\left(\left.\alpha\right|_{T_{1}}\right) \cong D^{2} \times S^{1}$ for any $\alpha \in \mathcal{A}_{2, j}$.

(2) $\mathcal{A}=\mathcal{A}_{1} \cup\left(\bigcup_{j=1}^{k} \mathcal{A}_{2, j}\right)$.

We will denote the component of $X$ cut open along $F_{j}$ that does not contain $X_{1}$ by $N_{j}$ and $\partial X \cap N_{j}$ by $\mathcal{T}_{j}$; to avoid the situation $N_{j}=\varnothing$, if $F_{j} \subset \partial X$ we push it slightly into the interior so that $N_{j} \cong T^{2} \times[0,1]$ in this case. Note that $\partial N_{j}=F_{j} \cup \mathcal{T}_{j}$.

Every $\alpha \in \mathcal{A}_{2, j}$ induces the multislope on $\partial N_{j}$, which we will denote by $\alpha_{j}$, defined by the slope of the meridian of the solid torus $X(\alpha)_{j}^{+}\left(\left.\alpha\right|_{T_{1}}\right)$ on $F_{j}$ and the restriction $\left.\alpha\right|_{\mathcal{T}}$ on $\mathcal{T}_{j}$. We show that the following two conditions hold:

(1) $N_{j}\left(\alpha_{j}\right) \cong S^{3}$ : by construction $N_{j}\left(\alpha_{j}\right) \cong X(\alpha)$, and by assumption $X(\alpha) \cong S^{3}$.

(2) Let $\alpha_{j}^{\prime} \subset_{\mathrm{pf}} \alpha_{j}$ be a partial filling for which $\left.\alpha_{j}^{\prime}\right|_{F_{j}}=\infty$. Then $N_{j}\left(\alpha_{j}^{\prime}\right) \not D^{2} \times S^{1}$ : assume for a contradiction that $N_{j}\left(\alpha_{j}^{\prime}\right) \cong D^{2} \times S^{1}$. Let $T_{i}$ be the component of $\partial N_{j} \cap X$ for which $\left.\alpha_{j}^{\prime}\right|_{T_{i}}=\infty$. Let $\alpha^{\prime} \subset_{\mathrm{pf}} \alpha$ be the partial filling giving by setting $\left.\alpha^{\prime}\right|_{T_{1}}$ and $\left.\alpha^{\prime}\right|_{T_{i}}$ to $\infty$. Then

$$
\begin{aligned}
X\left(\alpha^{\prime}\right) & \cong X(\alpha)_{j}^{+} \cup_{F_{j}} N_{j}\left(\alpha_{j}^{\prime}\right) \\
& \cong T^{2} \times[0,1] \cup_{F_{j}} T^{2} \times[0,1] \\
& \cong T^{2} \times[0,1],
\end{aligned}
$$

violating assumption (2) of the theorem.

Thus the assumptions of the theorem are satisfied by $N_{j}$ and $\left\{\alpha_{j} \mid \alpha \in \mathcal{A}_{2, j}\right\}$. By Lemma 5.6 we have $\left|T\left(N_{j}\right)\right|<|T(X)|$. By induction, $\left\{\left.\alpha_{j}\right|_{F_{j}}\right\}$ is bounded. For each $\alpha \in \mathcal{A}_{2, j},\left.\alpha\right|_{T_{1}}$ is the image of $\left.\alpha_{j}\right|_{F_{j}}$ under the projection induced by the product structure $X(\alpha)_{j}^{+} \cong T^{2} \times[0,1]$. By the $T^{2} \times[0,1]$ cosmetic surgery theorem (Theorem 6.1), the union of the images of $\left\{\left.\alpha_{j}\right|_{F_{j}}\right\}$ under the projections given by all possible fillings of $\partial X(\alpha)_{j}^{+} \backslash\left(T_{1} \cup F_{j}\right)$ for which $X(\alpha)_{j}^{+} \cong T^{2} \times[0,1]$ is a bounded set of slopes of $T_{1}$ that we will denote by $B_{2, j}$. We conclude that $\left.\alpha\right|_{T_{1}} \in B_{2, j}$. This completes Case 2.

Since $\mathcal{A}=\mathcal{A}_{1} \cup\left(\bigcup_{j} \mathcal{A}_{2, j}\right)$ we have

$$
\left\{\left.\alpha\right|_{T_{1}} \mid \alpha \in \mathcal{A}\right\} \subset B_{1} \cup\left(\bigcup_{j} B_{2, j}\right) .
$$

The theorem follows for JSJ manifolds, and we assume from now on that $X$ is irreducible, and not Seifert fibered, sol, or JSJ. Thus $X$ is hyperbolic. 
Assume that $\boldsymbol{X}$ is hyperbolic Since $S^{3}$ is not hyperbolic any $\alpha \in \mathcal{A}$ admits a minimally nonhyperbolic partial filling. Up to finite ambiguity we fix a minimally nonhyperbolic filling which we will denote by $\alpha^{\prime}$. If $\left.\alpha^{\prime}\right|_{T_{1}} \neq \infty$ then for any $\alpha$ with $\alpha^{\prime} \subset_{\mathrm{pf}} \alpha,\left.\alpha\right|_{T_{1}}=\left.\alpha^{\prime}\right|_{T_{1}}$ is in the finite (and hence bounded) set $\left\{\left.\alpha^{\prime}\right|_{T_{1}}\right\}$. Otherwise, any $\alpha$ with $\alpha^{\prime} \subset_{\mathrm{pf}} \alpha$ factors through $X\left(\alpha^{\prime}\right)$ as

$$
X \stackrel{\alpha^{\prime}}{\longrightarrow} X\left(\alpha^{\prime}\right) \stackrel{\left.\alpha\right|_{\partial X\left(\alpha^{\prime}\right)}}{\longrightarrow} X\left(\alpha^{\prime}\right)\left(\left.\alpha\right|_{\partial X\left(\alpha^{\prime}\right)}\right)
$$

It is straightforward to see that we can apply induction to $X\left(\alpha^{\prime}\right)$ and $\left\{\left.\alpha\right|_{\partial X\left(\alpha^{\prime}\right)} \mid \alpha \in \mathcal{A}\right\}$, showing that $\left\{\left.\left.\alpha\right|_{\partial X\left(\alpha^{\prime}\right)}\right|_{T_{1}} \mid \alpha \in \mathcal{A}\right\}$ is bounded. Thus for any $\alpha$ with $\alpha^{\prime} \subset_{\mathrm{pf}} \alpha$, we have that $\left.\alpha\right|_{T_{1}}=\left.\left.\alpha\right|_{\partial X\left(\alpha^{\prime}\right)}\right|_{T_{1}}$ is in this bounded set.

This completes the proof in this final case.

\section{Proof of Theorem 1.1}

In this section we prove Theorem 1.1 assuming the results of the next three sections. We decided to present the proof before Sections 12-14 in order to help the reader understand the motivation behind the exact statements proved in those sections.

As in the statement of the theorem, let $M$ be a hyperbolic manifold such that $\partial M$ is a single torus that we will denote by $T$, and let $V>0$ be a fixed number. Consider a slope $\beta$ on $T$ such that $\operatorname{Link} \operatorname{Vol}(M(\beta))<V$. By the structure theorem of [19], there exist finitely many covers $\phi: X \rightarrow E$, with $X$ and $E$ hyperbolic, such that the following diagram commutes:

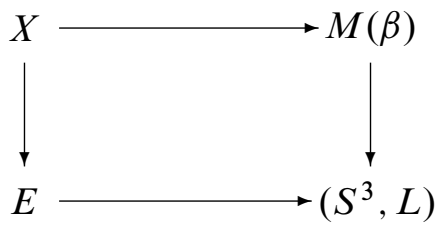

Here, the horizontal arrows represent inclusions induced by fillings and the vertical arrows represent covering projections; $\phi: X \rightarrow E$ is an unbranched covering projection between hyperbolic manifolds and $\hat{\phi}: M(\beta) \rightarrow S^{3}$ is a branched cover realizing $\operatorname{Link\operatorname {Vol}}(M(\beta))$. Up to finite ambiguity we fix one cover $\phi: X \rightarrow E$. We will denote the components of $\partial X$ by $T_{1}, \ldots, T_{n}$.

Case 1 We first consider fillings $X \rightarrow M(\beta)$ that do not factor though $M$, that is, slopes $\beta$ for which there exist a multislope $\alpha$ of $\partial X$ fulfilling the following two conditions:

(1) $X(\alpha) \cong M(\beta)$.

(2) There is no $\alpha^{\prime} \subset_{\text {pf }} \alpha$ such that $X\left(\alpha^{\prime}\right) \cong M$. 
In Section 12 we prove Theorem 12.1, showing that the set of slopes $\beta$ that arise in this way, which we will denote by $B_{1}$, is bounded. We remark that this is a general fact about fillings $X \rightarrow M(\beta)$ and does not use the covers $\phi: X \rightarrow E$ and $\widehat{\phi}: M(\beta) \rightarrow S^{3}$.

Case 2 We next consider fillings $X(\alpha) \cong M(\beta)$ that do factor though $M$, that is, fillings that admit a partial filling $M$. Up to finite ambiguity, we may assume that the component of $\partial X$ that corresponds to $\partial M$ is $T_{1}$. Thus we are considering multislopes $\alpha$ such that the diffeomorphism $X(\alpha) \rightarrow M(\beta)$ induces, by restriction, a diffeomorphism

$$
X\left(\left.\alpha\right|_{T_{2}, \ldots, T_{n}}\right) \rightarrow M .
$$

Denote the components of $\partial E$ by $T_{1}^{\prime}, \ldots, T_{m}^{\prime}$. By renumbering $T_{1}^{\prime}, \ldots, T_{m}^{\prime}$ if necessary we may assume that $T_{1}^{\prime}=\phi\left(T_{1}\right)$. The diagram above implies that $\alpha$ induces a multislope of $\partial E$, which we will denote as $\alpha^{E}=\left(\alpha_{1}^{E}, \ldots, \alpha_{m}^{E}\right)$. In Case 2 we only consider fillings on $E$ that do not factor through $T^{2} \times[0,1]$; more precisely, multislopes $\alpha^{E}$ fulfilling the following condition:

$$
E\left(\alpha^{\prime}\right) \not T^{2} \times[0,1] \text { for any } \alpha^{\prime} \subset_{\mathrm{pf}} \alpha^{E} \text { with }\left.\alpha^{\prime}\right|_{T_{1}^{\prime}}=\infty .
$$

In that case, the strategy is as follows: applying the $S^{3}$ cosmetic surgery theorem (Theorem 10.1) we see that the possibilities for $\alpha_{1}^{E}$ are bounded; the covering projection $\phi: X \rightarrow E$ induces a bilipschitz bijection between the slopes of $T_{1}^{\prime}$ and those of $T_{1}$ (Lemma 3.4); hence the possibilities for slopes on $T_{1}$ are bounded. The argument is worked out in detail in Section 13.

Case 3 The last and most exciting case is when the filling of $X$ factors through $M$ and the filling of $E$ factors through $T^{2} \times[0,1]$. The proof in this case is given in Section 14. Again, we conclude that $\left\{\left.\alpha\right|_{T_{1}}\right\}$ is bounded.

Assuming the results of the following sections, we deduce Theorem 1.1 as follows:

Proof of Theorem 1.1 Let $\mathcal{A}$ be the set of all multislopes of $\partial X$ such that for each $\alpha \in \mathcal{A}$ there is a slope $\beta$ of $\partial M$ with $X(\alpha) \cong M(\beta)$.

Since $M$ is hyperbolic there is a finite set of slopes of $\partial M$, which we will denote by $B_{F}^{\prime}$, such that $M(\beta)$ is hyperbolic for any $\beta \notin B_{F}^{\prime}$ and the core of the attached solid torus is its unique shortest geodesic (Lemma 2.7). Let $B_{F}$ be the set of slopes of $T$ given by

$$
B_{F}=\left\{\beta \mid M(\beta) \cong M\left(\beta^{\prime}\right) \text { for some } \beta^{\prime} \in B_{F}^{\prime}\right\} .
$$

Since $M$ is hyperbolic and $|\partial M|=1$, no manifold is obtained by filling infinitely many distinct slopes of $\partial M$; hence $B_{F}$ is finite. We will only consider multislopes 
$\alpha \in \mathcal{A}$ for which $X(\alpha) \cong M(\beta)$ for $\beta \notin B_{F}$. To avoid overly complicated notation we will not rename $\mathcal{A}$.

We now consider Cases 2 and 3. We will denote by $\mathcal{A}_{2,3}$ the multislopes of $\mathcal{A}$ in these cases, so that every $\alpha \in \mathcal{A}_{2,3}$ admits a partial filling $\alpha^{\prime} \subset_{\text {pf }} \alpha$ with $X\left(\alpha^{\prime}\right) \cong M$. Up to finite ambiguity and renumbering we may assume that $\alpha^{\prime}=\left.\alpha\right|_{T_{2}, \ldots, T_{n}}$. We will denote the set of restrictions $\left\{\left.\alpha\right|_{T_{1}} \mid \alpha \in \mathcal{A}_{2,3}\right\}$ as $B_{M}^{\prime}$. By Propositions 13.1 and 14.6 and Lemma 14.1 (see also Remark 14.7), $B_{M}^{\prime}$ is bounded.

Let $\beta$ be a slope of $\partial M$ such that $M(\beta) \cong X(\alpha)$ for some $\alpha \in \mathcal{A}_{2,3}$. We will consider $X(\alpha)$ as $X\left(\left.\alpha\right|_{T_{2}, \ldots, T_{n}}\right)\left(\left.\alpha\right|_{T_{1}}\right)$, the manifold obtained by filling $X\left(\left.\alpha\right|_{T_{2}, \ldots, T_{n}}\right)$ along slope $\left.\alpha\right|_{T_{1}}$. Then we see that $X\left(\left.\alpha\right|_{T_{2}, \ldots, T_{n}}\right) \cong M$ and

$$
X\left(\left.\alpha\right|_{T_{2}, \ldots, T_{n}}\right)\left(\left.\alpha\right|_{T_{1}}\right) \cong M(\beta) .
$$

Let $f: X\left(\left.\alpha\right|_{T_{2}, \ldots, T_{n}}\right) \rightarrow M$ be a diffeomorphism. Denote the image of $\left.\alpha\right|_{T_{1}}$ under $f$ by $\alpha^{M}$ and the image of $B_{M}^{\prime}$ under $f_{\left.\alpha\right|_{T_{2}}, \ldots, T_{n}}$ by $B_{M, f}$; note that $\alpha^{M} \in B_{M, f}$. Let $i$ be the isometric involution on the slopes of $T$ given by Lemma 3.8; we will denote $B_{M, f} \cup i\left(B_{M, f}\right)$ by $B_{M}$. Clearly $B_{M}$ is bounded and $\alpha^{M} \in B_{M}$.

Since $M\left(\alpha^{M}\right) \cong X\left(\left.\alpha\right|_{T_{2}, \ldots, T_{n}}\right)\left(\left.\alpha\right|_{T_{1}}\right)$ and $M(\beta)$ are diffeomorphic, by Mostow rigidity there is an isometry $f: M\left(\alpha^{M}\right) \rightarrow M(\beta)$. Since $\beta \notin B_{F}$, the cores of the attached solid tori are the shortest geodesics of $M\left(\alpha^{M}\right)$ and $M(\beta)$. Thus $f$ carries the core of the solid torus attached to $M\left(\alpha^{M}\right)$ to the core of the solid torus attached to $M(\beta)$ and hence by restriction $f$ induces an isometry $M \rightarrow M$ that maps $\alpha^{M}$ to $\beta$. By Lemma 3.8, $\alpha^{M}=\beta$ or $\alpha^{M}=i(\beta)$. Since $\alpha^{M} \in B_{M}$ and $B_{M}=i\left(B_{M}\right)$, we conclude that $\beta \in B_{M}$.

Recall that we denoted the set of slopes of $\partial M$ that is realized in Case 1 by $B_{1}$; in Theorem 12.1 we show that $B_{1}$ is bounded. Combining all the possibilities we see that

$$
\beta \in B_{M} \cup B_{1} \cup B_{F} .
$$

Theorem 1.1 follows.

\section{Case 1: fillings of $X$ that do not factor through $M$}

In this section we consider two manifolds, denoted by $X$ and $M$, where $M$ is a one-cusped hyperbolic manifold. If $\alpha^{\prime}$ is a multislope of $X$ such that $X\left(\alpha^{\prime}\right) \cong M$, then (trivially) for any slope $\beta$ of $\partial M$ there is a multislope $\alpha$ such that $\alpha^{\prime} \subset_{\text {pf }} \alpha$ and $X(\alpha) \cong M(\beta)$. In Theorem 12.1 we show that if one considers only multislopes $\alpha$ that do not admit $\alpha^{\prime} \subset_{\text {pf }} \alpha$ with $X\left(\alpha^{\prime}\right) \cong M$, then the set of slopes of $\partial M$ that give 
rise to manifolds that are also obtained by filling $X$ is bounded. This is purely a result about filling and is independent of the covers considered in this paper. The precise statement is:

Theorem 12.1 Let $X$ be a compact orientable connected manifold so that $\partial X$ consists of tori and $M$ a hyperbolic manifold with $\partial M$ a single torus. Let $\mathcal{A}$ be a set of multislopes of $\partial X$ and $\mathcal{B}$ a set of slopes of $\partial M$ fulfilling the following two conditions:

(1) For every $\beta \in \mathcal{B}$ there is a multislope $\alpha \in \mathcal{A}$ so that $X(\alpha) \cong M(\beta)$.

(2) For every $\alpha \in \mathcal{A}$ and every $\alpha^{\prime} \subset_{\mathrm{pf}} \alpha, X(\alpha) \not M$.

Then $\mathcal{B}$ is bounded.

Proof We denote $\partial M$ by $T$. By Thurston's Dehn surgery theorem (see Lemma 2.7) we may fix an $\epsilon>0$ and a finite set of slopes of $T$, which we will denote by $B_{f}^{\prime}$, such that for every $\beta \notin B_{f}^{\prime}$ the following three conditions hold:

(1) $M(\beta)$ is hyperbolic.

(2) The core of the attached solid torus, which we will denote by $\gamma$, is a geodesic and $l(\gamma)<\epsilon$.

(3) Any geodesic $\delta \subset M(\beta)$ with $l(\delta)<\epsilon$ is a power of $\gamma$.

Since no manifold is obtained by filling along infinitely many distinct slopes of $M$, the set $B_{f}=\left\{\beta \mid M(\beta) \cong M\left(\beta^{\prime}\right)\right.$ for some $\left.\beta^{\prime} \in B_{f}^{\prime}\right\}$ is finite. For the remainder of the proof we only consider $\beta \notin B_{f}$. Accordingly, we remove the multislopes $\alpha \in \mathcal{A}$ for which $X(\alpha) \cong M(\beta)$ for $\beta \in B_{f}$. To avoid overly complicated notation we do not rename $\mathcal{A}$ and $\mathcal{B}$.

We induct on $|T(X)|$.

Assume that $X$ is Seifert fibered or sol Then $X$ cannot be filled to give a hyperbolic manifold.

We assume from now on that $X$ is not Seifert fibered or sol.

Assume that $X$ is reducible Let $X_{1}, \ldots, X_{n}$ be the factors of the prime decomposition of $X$. For every $\alpha \in \mathcal{A}$ we will denote the restriction $\left.\alpha\right|_{\partial X_{i}}$ by $\alpha_{i}$. By considering the image of decomposing spheres for $X$ in $X(\alpha)$ we see that for every $\alpha \in \mathcal{A}$ we have

$$
X(\alpha)=X_{1}\left(\alpha_{1}\right) \# \cdots \# X_{n}\left(\alpha_{n}\right) .
$$


For $1 \leq i \leq n$ we will denote by $\mathcal{A}_{i} \subset \mathcal{A}$ the multislopes $\alpha$ for which $X_{i}\left(\alpha_{i}\right) \cong M(\beta)$ (for some $\beta \in \mathcal{B}$ ) and for $i^{\prime} \neq i, X_{i^{\prime}}\left(\alpha_{i^{\prime}}\right) \cong S^{3}$. Since $\beta \notin B_{f}, X(\alpha)$ is hyperbolic and hence prime. Thus, by (1),

$$
\mathcal{A}=\bigcup_{i=1}^{n} \mathcal{A}_{i} .
$$

Fix $1 \leq i \leq n$. Suppose, for a contradiction, that for some $\alpha \in \mathcal{A}_{i}$ there is $\alpha_{i}^{\prime} \subset_{\mathrm{pf}} \alpha_{i}$ such that $X_{i}\left(\alpha_{i}^{\prime}\right) \cong M$. Then there is a unique component $T$ of $\partial X_{i}$ for which $\left.\alpha_{i}^{\prime}\right|_{T}=\infty$. Let $\alpha^{\prime} \subset_{\mathrm{pf}} \alpha$ be the partial filling defined by setting $\left.\alpha^{\prime}\right|_{T}=\infty$ and $\left.\alpha^{\prime}\right|_{T^{\prime}}=\left.\alpha\right|_{T^{\prime}}$ for any other component $T^{\prime}$ of $\partial X$. Then, by (1),

$$
\begin{aligned}
X\left(\alpha^{\prime}\right) & \cong X_{i}\left(\left.\alpha^{\prime}\right|_{\partial X_{i}}\right) \#\left(\#_{i^{\prime} \neq i} X_{i^{\prime}}\left(\left.\alpha^{\prime}\right|_{\partial X_{i^{\prime}}}\right)\right) \\
& \cong X_{i}\left(\alpha_{i}^{\prime}\right) \#\left(\#_{i^{\prime} \neq i} X_{i^{\prime}}\left(\alpha_{i^{\prime}}\right)\right) \\
& \cong M \#\left(\#_{i^{\prime} \neq i} S^{3}\right) \\
& \cong M
\end{aligned}
$$

(Here we used that $\left.X_{i^{\prime}}\left(\alpha_{i^{\prime}}\right)\right) \cong S^{3}$, which holds by the definition of $\mathcal{A}_{i}$.) This contradicts the assumptions of the theorem. Hence $X_{i}$ and $\left\{\alpha_{i} \mid \alpha \in \mathcal{A}_{i}\right\}$ fulfill the assumptions of the theorem. Since $X_{i}$ corresponds to a direct descendant of the root of $T(X)$, we have $\left|T\left(X_{i}\right)\right|<|T(X)|$. We will denote by $B_{i}$ the set of slopes of $T$ such that for every $\beta \in B_{i}$ there is $\alpha_{i} \in \mathcal{A}_{i}$ with $M(\beta) \cong X_{i}\left(\alpha_{i}\right)$. By induction, $B_{i}$ is bounded. As $i$ was arbitrary, we obtain bounded sets $B_{1}, \ldots, B_{n}$. Since $\mathcal{A}=\bigcup_{i=1}^{n} \mathcal{A}_{i}$,

$$
\mathcal{B} \subset B_{f} \cup\left(\bigcup_{i=1}^{n} B_{i}\right) .
$$

The theorem follows for reducible manifolds.

We assume from now on that $X$ is irreducible and not Seifert fibered or sol.

Assume that $X$ is hyperbolic Since there is no lower bound on the length of geodesics in $\{M(\beta) \mid \beta \in \mathcal{B}\}$, Theorem 9.1 does not apply directly; however, the proof here is similar to the proof of that theorem, where more details can be found. We start with:

Claim There are only finitely many totally hyperbolic fillings in $\mathcal{A}$.

When proving the claim we may obviously assume that $\mathcal{A}$ is infinite. Let $\left\{\alpha^{j}\right\}_{j \in \mathbb{N}} \subset \mathcal{A}$ be an infinite set. We will prove the claim by showing that some multislope of $\left\{\alpha^{j}\right\}_{j \in \mathbb{N}}$ is not totally hyperbolic. We denote the components of $\partial X$ by $T_{1}, \ldots, T_{n}$ and $\left.\alpha^{j}\right|_{T_{i}}$ by $\alpha_{i}^{j}$. After subsequencing and reordering if necessary we assume as we may that for some $0 \leq k \leq n$ the following hold: 
(1) $\alpha_{i}^{j} \neq \alpha_{i}^{j^{\prime}}$ and $\alpha_{i}^{j} \neq \infty$ for every $1 \leq i \leq k$ and every $j \neq j^{\prime}$.

(2) $\alpha_{i}^{j}=\alpha_{i}^{j^{\prime}}$ for every $k+1 \leq i \leq n$ and every $j, j^{\prime}$.

Since $\left\{\alpha^{j}\right\}_{j \in \mathbb{N}}$ is infinite, $k \geq 1$. Let $\alpha^{\prime}=\left(\infty, \ldots, \infty, \alpha_{k+1}^{j}, \ldots, \alpha_{n}^{j}\right)$; by construction $\alpha^{\prime}$ does not depend on $j$. We claim that $X\left(\alpha^{\prime}\right)$ is not hyperbolic. Assume, for a contradiction, that it is. Then for any $l>0$ we may choose $j$ such that the slopes $\alpha_{1}^{j}, \ldots, \alpha_{k}^{j}$ are all longer than $l$ (where the lengths are measured in the Euclidean metrics induced on the boundary components after some truncation of the cusps). By Thurston's Dehn surgery theorem, if $l$ is sufficiently large then $X\left(\alpha^{\prime}\right)\left(\alpha_{1}^{j}, \ldots, \alpha_{k}^{j}\right)$ is hyperbolic and admits at least $k$ geodesics that are all shorter than $\epsilon$; since $M(\beta)$ admits only one such geodesic, $k=1$. Thus $X\left(\alpha^{\prime}\right)$ and $M$ are one-cusped hyperbolic manifolds that admit infinitely many diffeomorphic fillings. A standard application of Mostow rigidity shows that $X\left(\alpha^{\prime}\right) \cong M$. Since $\alpha^{\prime} \subset_{\mathrm{pf}} \alpha^{j}$ this contradicts the second assumption of the theorem, showing that $X\left(\alpha^{\prime}\right)$ is not hyperbolic. Hence $\alpha^{\prime} \subset_{\mathrm{pf}} \alpha^{j}$ is a nonhyperbolic partial filling, showing that $\alpha^{j}$ is not totally hyperbolic. Hence there are only finitely many totally hyperbolic fillings, as claimed.

By the claim there are only finitely many $\alpha \in \mathcal{A}$ that are totally hyperbolic. We remove these multislopes from $\mathcal{A}$ and remove from $\mathcal{B}$ the finite set of slopes $\beta$ for which $M(\beta) \cong X(\alpha)$ only for totally hyperbolic fillings; to avoid overly complicated notation we do not rename $\mathcal{A}$ and $\mathcal{B}$. Hence every multislope of $\mathcal{A}$ admits a minimally nonhyperbolic partial filling. Up to finite ambiguity we fix one minimally nonhyperbolic partial filling of $X$ and denote it by $\alpha^{\prime}$. By definition of partial filling, if $\alpha^{\prime} \subset_{\mathrm{pf}} \alpha$ then $X(\alpha)$ is obtained by filling $X\left(\alpha^{\prime}\right)$ along slopes $\left.\alpha\right|_{\partial X\left(\alpha^{\prime}\right)}$. By assumption, $\alpha$ does not admit a partial filling that yields $M$; hence the same holds for $\left.\alpha\right|_{\partial X\left(\alpha^{\prime}\right)}$. Since $X\left(\alpha^{\prime}\right)$ corresponds to a direct descendant of the root of $T(X)$, we have $\left|T\left(X\left(\alpha^{\prime}\right)\right)\right|<|T(X)|$. By induction the set of slopes of $T$

$$
\left\{\beta \mid M(\beta) \cong X\left(\alpha^{\prime}\right)\left(\left.\alpha\right|_{\partial X\left(\alpha^{\prime}\right)}\right), \alpha \in \mathcal{A}\right\}
$$

is bounded. The theorem follows.

We assume from now on that $X$ is irreducible, and not Seifert fibered, sol, or hyperbolic.

Assume that $\boldsymbol{X}$ is JSJ By Proposition 5.2 for every $\beta \in \mathcal{B}, M(\beta)$ is obtained by filling some component of the torus decomposition of $X$. Up to finite ambiguity we fix such component which we will denote by $X_{1}$ and consider only slopes $\beta$ (and corresponding multislopes $\alpha$ ) such that $M(\beta)$ is obtained by filling $X_{1}$; to avoid overly complicated notation we do not rename $\mathcal{A}$ and $\mathcal{B}$. Recall that in Proposition 5.2 for every $\alpha \in \mathcal{A}$ we constructed a multislope for $X_{1}$, which we will call the induced multislope and denote by $\alpha_{1}$, such that $M(\beta) \cong X_{1}\left(\alpha_{1}\right)$. We will denote the set of 
induced multislopes thus obtained by $\mathcal{A}_{1}$; thus for every $\beta \in \mathcal{B}$ there is $\alpha_{1} \in \mathcal{A}_{1}$ such that $M(\beta) \cong X_{1}\left(\alpha_{1}\right)$.

Let $\mathcal{A}_{1}^{+}$be the multislopes $\alpha_{1} \in \mathcal{A}_{1}$ for which there is no $\alpha_{1}^{\prime} \subset_{\mathrm{pf}} \alpha_{1}$ such that $X_{1}\left(\alpha_{1}^{\prime}\right) \cong M$. Then $X_{1}$ and $\mathcal{A}_{1}^{+}$fulfill the assumptions of the theorem. By Lemma 5.6, $\left|T\left(X_{1}\right)\right|<|T(X)|$. By induction, the set

$$
B_{1}^{+}=\left\{\beta \mid M(\beta) \cong X_{1}\left(\alpha_{1}\right) \text { for some } \alpha_{1} \in \mathcal{A}_{1}^{+}\right\}
$$

is bounded. We will return to $B_{1}^{+}$at the end of the proof.

We will denote $\mathcal{A}_{1} \backslash \mathcal{A}_{1}^{+}$by $\mathcal{A}_{1}^{-}$. Then every $\alpha_{1} \in \mathcal{A}_{1}^{-}$admits $\alpha_{1}^{\prime} \subset_{\mathrm{pf}} \alpha_{1}$ such that $X_{1}\left(\alpha_{1}^{\prime}\right) \cong M$. Up to finite ambiguity we fix one boundary component of $\partial X_{1}$, which we will denote by $F$, and consider only the multislopes $\alpha_{1} \in \mathcal{A}_{1}^{-}$for which $\left.\alpha_{1}^{\prime}\right|_{F}=\infty$ (in other words, $F \subset \partial X_{1}$ corresponds to $T$ after filling along $\alpha_{1}^{\prime}$ ). Note that $F \not \subset \partial X$, for otherwise $\alpha_{1}$ would correspond to a multislope $\alpha \in \mathcal{A}$ that admits $\alpha^{\prime} \subset_{\mathrm{pf}} \alpha$ for which $X\left(\alpha^{\prime}\right) \cong M$, contradicting the assumptions of the theorem. We will denote the components of $X$ cut open along $F$ by $X^{+}$and $X^{-}$, with $X_{0} \subset X^{+}$.

In the remainder of the proof we work directly with the set of multislopes of $\mathcal{A}$ that induce multislopes of $\mathcal{A}_{1}^{-}$fulfilling the assumptions above, which we will denote by $\mathcal{A}^{-} \subset \mathcal{A}$. Given $\alpha \in \mathcal{A}^{-}$, we will denote the multislope $\left.\alpha\right|_{\partial X^{+}}$induced on $\partial X^{+}$ by $\alpha^{+}$and the multislope $\left.\alpha\right|_{\partial X^{-}}$induced on $\partial X^{-}$by $\alpha^{-}$. By the defining assumption of $\mathcal{A}_{1}^{-}$we have $X^{+}\left(\alpha^{+}\right) \cong M$, and by Proposition 5.2 either $X^{-}\left(\alpha^{-}\right) \cong D^{2} \times S^{1}$ or $X^{-}\left(\alpha^{-}\right) \cong E(K)$ for a nontrivial knot $K \subset S^{3}$. But if the latter occurred, $F$ would be an incompressible torus in $M(\beta)$, which is absurd as $M(\beta)$ is hyperbolic. Thus $X^{-}\left(\alpha^{-}\right) \cong D^{2} \times S^{1}$. We will denote the slope defined by the meridian of $X^{-}\left(\alpha^{-}\right)$ on $F$ by $\mu_{F}$ and consider $X(\alpha)$ as $X^{+}\left(\alpha^{+}\right)\left(\mu_{F}\right)$, the manifold obtained by filling $X^{+}\left(\alpha^{+}\right)$along slope $\mu_{F}$.

Let $f: X^{+}\left(\alpha^{+}\right)\left(\mu_{F}\right) \rightarrow M(\beta)$ be a diffeomorphism. Then, since we assumed that $\beta \notin B_{f}$ (recall the definition of $B_{f}$ in the beginning of the proof), the cores of the solid tori attached to $X^{+}\left(\alpha^{+}\right)$and $M$ are the unique shortest geodesics in $X^{+}\left(\alpha^{+}\right)\left(\mu_{F}\right)$ and $M(\beta)$. By Mostow rigidity we may assume that $f$ is an isometry, and thus $f$ carries the core of the solid torus attached to $X^{+}\left(\alpha^{+}\right)$to the core of the solid torus attached to $M$. The restriction of $f$ induces a diffeomorphism which we will denote by $f^{+}: X^{+}\left(\alpha^{+}\right) \rightarrow M$. Note that $f^{+}$maps $\mu_{F}$ to $\beta$.

Turning our attention to the solid torus $X^{-}$, we claim that for every $\alpha \in \mathcal{A}^{-}$, the induced multislope $\alpha^{-}$satisfies the following conditions: 
(1) $X^{-}\left(\alpha^{-}\right) \cong D^{2} \times S^{1}$ : this was established above.

(2) For any partial filling $\widehat{\alpha} \subset_{\mathrm{pf}} \alpha^{-}, X^{-}(\widehat{\alpha}) \not T^{2} \times[0,1]$ : otherwise, let $\alpha^{\prime} \subset_{\mathrm{pf}} \alpha$ be the partial filling defined by setting $\left.\alpha^{\prime}\right|_{\partial X^{-}}=\hat{\alpha}$ and $\left.\alpha^{\prime}\right|_{\partial X^{+}}=\left.\alpha\right|_{\partial X^{+}}$. Then we have

$$
X\left(\alpha^{\prime}\right) \cong X^{-}(\widehat{\alpha}) \cup_{F} X^{+}\left(\left.\alpha^{+}\right|_{\partial X^{+}}\right) \cong\left(T^{2} \times[0,1]\right) \cup_{F} M \cong M,
$$

contradicting the assumption of the theorem.

Thus $X^{-}$and the multislopes $\mathcal{A}_{1}^{-}$fulfill the requirements of the solid torus cosmetic surgery theorem (Theorem 7.1), showing that the set of meridians of the solid tori $\left\{X^{-}\left(\alpha^{-}\right) \mid \alpha^{-} \in \mathcal{A}_{1}^{-}\right\}$, which we will denote by $B^{-}$, is bounded.

By the discussion above, if $X(\alpha) \cong M(\beta)$ then $\beta$ is the image of $\mu_{F} \in B^{-}$under the diffeomorphism denoted above by $f^{+}: X^{+}\left(\alpha^{+}\right) \rightarrow M$. We will denote the image of $B^{-}$under $f^{+}$by $B_{f}^{-}$. By Lemma 3.8 there is an isometric involution $i$ on the slopes of $F$ such that if $g^{+}: X^{+}\left(\alpha^{+}\right) \rightarrow M$ is a diffeomorphism, the image of $B^{-}$ under $g^{+}$is either $B_{f^{+}}^{-}$or $i\left(B_{f^{+}}^{-}\right)$. We will denote $B_{f}^{-} \cup i\left(B_{f^{+}}^{-}\right)$by $B_{M}$. Clearly, $B_{M}$ is bounded. The theorem follows for JSJ manifolds, as $\mathcal{B} \subset B_{f} \cup B_{1}^{+} \cup B_{M}$.

This completes the proof of Theorem 12.1.

\section{Case 2: fillings of $E$ that do not factor through $T^{2} \times[0,1]$}

In this section we prove the following proposition, which is used in Case 2 of the proof of Theorem 1.1:

Proposition 13.1 Let $X$ and $E$ be compact orientable connected manifolds with toral boundary and $\phi: X \rightarrow E$ a branched cover such that $\left.\phi\right|_{\partial X}: \partial X \rightarrow \partial E$ is a cover. Let $\widehat{\mathcal{A}}$ be a set of multislopes of $X$ such that every $\widehat{\alpha} \in \hat{\mathcal{A}}$ induces a multislope on $\partial E$ which we will denote by $\alpha^{E}$. Fix a component of $\partial X$ (which we will denote by $T_{1}$ ) and denote $\phi\left(T_{1}\right)$ by $T_{1}^{\prime}$. Suppose that every $\alpha^{E}$ fulfills the following conditions:

(1) $E\left(\alpha^{E}\right) \cong S^{3}$.

(2) There does not exist $\alpha^{\prime} \subset_{\mathrm{pf}} \alpha^{E}$ fulfilling the following two conditions:

(a) $\left.\alpha^{\prime}\right|_{T_{1}^{\prime}}=\infty$.

(b) $E\left(\alpha^{\prime}\right) \cong T^{2} \times[0,1]$.

Then the set of restrictions $\left\{\left.\hat{\alpha}\right|_{T_{1}} \mid \widehat{\alpha} \in \widehat{\mathcal{A}}\right\}$ is bounded. 
Proof Let $\mathcal{A}_{E}$ be the set multislopes $\left\{\alpha^{E}\right\}$ above. By the $S^{3}$ cosmetic surgery theorem (Theorem 10.1), the set of restrictions $\left\{\left.\alpha^{E}\right|_{T_{1}^{\prime}} \mid \alpha^{E} \in \mathcal{A}_{E}\right\}$ is bounded. The set of restrictions $\left\{\left.\hat{\alpha}\right|_{T_{1}} \mid \widehat{\alpha} \in \widehat{\mathcal{A}}\right\}$ is contained in the image of $\left\{\left.\alpha^{E}\right|_{T_{1}^{\prime}} \mid \alpha^{E} \in \mathcal{A}_{E}\right\}$ under the bilipschitz bijection induced by $\phi$ (Lemma 3.4). The proposition follows.

\section{Case 3: fillings of $X$ that factor through $M$ and fillings of $E$ that factor through $T^{2} \times[0,1]$}

In this section we tackle Case 3, the final case of Theorem 1.1. Below we denote by $\mathcal{A}_{3}$ the multislopes in $\mathcal{A}$ that correspond to this case. After general analysis of the situation, we show that certain conditions must hold (up to finite ambiguity). These conditions are summarized in Lemma 14.5. Theorem 1.1 then follows from Proposition 14.6 and Lemma 14.1.

We begin by fixing our notation. Let $X, M(\beta), E, L, \phi$, and $\widehat{\phi}$ be as in the diagram in Section 11 (see page 3499). We will denote the multislope of $X$ that corresponds to the filling $X \rightarrow M(\beta)$ by $\alpha$ and the multislope of $E$ that corresponds to $E \rightarrow S^{3}$ by $\alpha^{E}$. By construction, $\alpha$ and $\alpha^{E}$ are corresponding multislopes (in the correspondence defined by the restriction of $\phi: X \rightarrow E$ to the boundary; recall Section 3A). Up to finite ambiguity we fix two components of $\partial E$ which we will denote by $T_{1}^{\prime}$ and $T_{2}^{\prime}$ (note that $|\partial E| \geq 2$ since $E \rightarrow S^{3}$ is assumed to factor through $T^{2} \times[0,1]$ ) and a component of $\phi^{-1}\left(T_{1}^{\prime}\right)$ which we will denote by $T_{1}$. We will denote the remaining components of $\partial X$ by $T_{2}, \ldots, T_{n}$ and the remaining components of $\partial E$ by $T_{3}^{\prime}, \ldots, T_{k}^{\prime}$. In the two preceding sections we have reduced the proof of Theorem 1.1 to multislopes $\alpha \in \mathcal{A}$ fulfilling the following conditions:

(1) $X(\alpha) \cong M(\beta)$ (for some slope $\beta$ of $\partial M$ ).

(2) $X\left(\left.\alpha\right|_{T_{2}, \ldots, T_{n}}\right) \cong M$.

(3) $E\left(\left.\alpha^{E}\right|_{T_{3}^{\prime}, \ldots, T_{k}^{\prime}}\right) \cong T^{2} \times[0,1]$.

Thus we obtain the following commutative diagram, where horizontal arrows represent inclusions induced by fillings, vertical arrows represent covering projections, $T^{2} \times[0,1]$ represents $E\left(\left.\alpha^{E}\right|_{T_{3}^{\prime}, \ldots, T_{k}^{\prime}}\right), \phi^{-1}\left(T^{2} \times[0,1]\right)$ is denoted by $X^{\prime}$, and $\left.\phi\right|_{X^{\prime}}$ is denoted by $\phi^{\prime}$ :

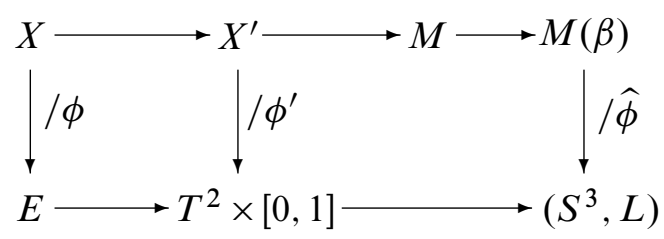


We will denote the set of all multislopes fulfilling these conditions by $\mathcal{A}_{3} \subset \mathcal{A}$. For $\alpha \in \mathcal{A}_{3}$, we will denote the restriction $\left.\alpha\right|_{T_{2}, \ldots, T_{n}}$ by $\hat{\alpha}$, and the set or restriction $\left\{\hat{\alpha} \mid \alpha \in \mathcal{A}_{3}\right\}$ by $\hat{\mathcal{A}}$.

Our goal is to show that the set of slopes induced on $T_{1}$ by restricting the multislopes $\mathcal{A}_{3}$ is bounded. However, in the course of the proof we sometimes end up with a bounded set of slopes of a different component of $\partial X$, say $T_{2}$. It will always be the case that $\phi\left(T_{2}\right)=T_{1}^{\prime}$ or $\phi\left(T_{2}\right)=T_{2}^{\prime}$. If $\phi\left(T_{2}\right)=T_{1}^{\prime}$, then the covering map $\phi$ induces a bijection between the slopes of $T_{2}$ and those of $T_{1}^{\prime}$, and a bijection between the slopes of $T_{1}^{\prime}$ and those of $T_{1}$; both bijections are bilipschitz maps of the Farey graph (Lemma 3.4). Composing the two bijections, we readily see a bounded set of slopes on $T_{1}$. If instead $\phi\left(T_{2}\right)=T_{2}^{\prime}$, let $B_{2}$ be the bounded set of slopes of $T_{2}$ and let $B_{2}^{\prime}$ be its image (in the slopes of $T_{2}^{\prime}$ ) under the bijection induced by $\phi$; by Lemma 3.4, $B_{2}^{\prime}$ is bounded. Since $E\left(\left.\alpha^{E}\right|_{T_{3}^{\prime}, \ldots, T_{k}^{\prime}}\right) \cong T^{2} \times[0,1]$, we can then project $B_{2}^{\prime}$ from $T_{2}^{\prime}$ to a set of slopes on $T_{1}^{\prime}$ using the product structure. As this set depends on $\alpha^{E}$, we denote it by $B_{1, \alpha}^{\prime}$. By the $T^{2} \times[0,1]$ cosmetic surgery theorem (Theorem 6.1), $\cup_{\alpha^{E}} B_{1, \alpha^{E}}^{\prime}$ is a bounded set of slopes on $T_{1}^{\prime}$. Using Lemma 3.4 once more, we obtain a bounded set of slopes on $T_{1}$.

We summarize this:

Lemma 14.1 With the notation as above, suppose that, for each component $T_{i}$ of $\phi^{-1}\left(T_{1}^{\prime} \cup T_{2}^{\prime}\right)$, there is a bounded set of slopes of $T_{i}$, that we will denote by $B_{i}$, such that for each $\alpha \in \mathcal{A}_{3}$, there is some component $T_{i}$ of $\phi^{-1}\left(T_{1}^{\prime} \cup T_{2}^{\prime}\right)$ with $\left.\alpha\right|_{T_{i}} \in B_{i}$.

Then $\left\{\left.\alpha\right|_{T_{1}} \mid \alpha \in \mathcal{A}_{3}\right\}$ is bounded.

Remark 14.2 It is unfortunate that this set up does not lend itself well to induction. There are many problems, and here is perhaps the best example: when considering a JSJ manifold $X$ we apply Proposition 5.2 and conclude that $M(\beta)$ is obtained by filling some component of the torus decomposition of $X$; however, the cover $\phi^{\prime}: X^{\prime} \rightarrow E^{\prime}$ is nowhere to be found. We must therefore first identify the essential information that is preserved in the inductive step. This is done in the following two lemmas:

Lemma 14.3 Any prime factor of $X^{\prime}$ has at least two boundary components.

Proof If $X^{\prime}$ is prime then the lemma follows from the fact that $T \times\{0\}$ and $T \times\{1\}$ (as components of $\left.\partial E\left(\left.\alpha^{E}\right|_{T_{3}^{\prime}, \ldots, T_{k}^{\prime}}\right) \cong T^{2} \times[0,1]\right)$ have at least one preimage each. Otherwise, let $S \subset X^{\prime}$ be a decomposing sphere that realizes the decomposition $X^{\prime}=X^{\prime \prime} \# X^{\prime \prime \prime}$, where $X^{\prime \prime}$ is a prime factor of $X^{\prime}$. We will prove the lemma by showing that $\left|\partial X^{\prime \prime}\right| \geq 2$. 
(1) Suppose that $\left|\partial X^{\prime \prime}\right|=0$. Equivalently, $X^{\prime \prime}$ is closed. Then $X^{\prime \prime}$ is a prime factor of $M$. But $M$ is a hyperbolic manifold and $\partial M \neq \varnothing$, and so it has no closed factors. Thus $\left|\partial X^{\prime \prime}\right| \neq 0$.

(2) Suppose that $\left|\partial X^{\prime \prime}\right|=1$. Without loss of generality we may assume that $\phi^{\prime}\left(\partial X^{\prime \prime}\right)=$ $T^{2} \times\{0\}$. We will denote the component of $X^{\prime}$ cut open along $S$ that corresponds to $X^{\prime \prime}$ by $X^{*}$; note that $\partial X^{*}$ has two components: a torus (which we can naturally identify with $\partial X^{\prime \prime}$ ) and a sphere (which we can naturally identify with $S$ ). Since $\pi_{2}\left(T^{2} \times[0,1]\right)$ is trivial, $\left.\phi^{\prime}\right|_{S}: S \rightarrow T^{2} \times[0,1]$ can be extended to a map from the closed ball $D$, which we will denote by $\phi^{\prime \prime}$. After a homotopy of $\phi^{\prime}$ if necessary, we assume as we may that for a sufficiently small fixed $\epsilon>0$ the following three conditions hold:

(a) $\phi^{\prime \prime}(D) \cap\left(T^{2} \times[0, \epsilon)\right)=\varnothing$.

(b) $\phi^{\prime \prime}(D) \cap\left(T^{2} \times(1-\epsilon, 1]\right)=\varnothing$.

(c) $\phi^{\prime}\left(X^{*}\right) \cap\left(T^{2} \times(1-\epsilon, 1]\right)=\varnothing$.

We paste $\phi^{\prime \prime}: D \rightarrow T^{2} \times[0,1]$ and $\phi^{\prime}: X^{*} \rightarrow T^{2} \times[0,1]$ to obtain a map which we will denote as $\psi: X^{\prime \prime}=X^{*} \cup_{S} D \rightarrow T^{2} \times[0,1]$. By conditions (a) and (b) above the following holds:

$$
\left.\psi\right|_{\psi^{-1}\left(T^{2} \times[0, \epsilon)\right)}=\left.\phi^{\prime}\right|_{\psi^{-1}\left(T^{2} \times[0, \epsilon)\right)} .
$$

Therefore $\left.\psi\right|_{\psi^{-1}\left(T^{2} \times[0, \epsilon)\right)}: \psi^{-1}\left(T^{2} \times[0, \epsilon)\right) \rightarrow T^{2} \times[0, \epsilon)$ is a cover and has nonzero degree. On the other hand, $\psi^{-1}\left(T^{2} \times(1-\epsilon, 1]\right)=\varnothing$ so the map

$$
\left.\psi\right|_{\psi^{-1}\left(T^{2} \times(1-\epsilon, 1]\right)}: \psi^{-1}\left(T^{2} \times(1-\epsilon, 1]\right) \rightarrow T^{2} \times(1-\epsilon, 1]
$$

has degree zero. This contradiction shows that $\left|\partial X^{\prime \prime}\right| \neq 1$.

In light of this lemma, we define:

Notation 14.4 We will denote the prime factor of $X^{\prime}$ that contains $T_{1}$ by $X^{\prime \prime}$ and the components of $\partial X^{\prime \prime} \backslash T_{1}$ by $\mathcal{T}$. Given $\alpha \in \mathcal{A}_{3}$, the link in $M$ consisting of the cores of the solid tori attached to $\mathcal{T}$ will be denoted by $\mathcal{L}_{\alpha}$, or simply $\mathcal{L}$ when no confusion may arise.

Next we prove:

Lemma 14.5 $\mathcal{L}$ has the following properties:

(1) $\mathcal{L} \neq \varnothing$.

(2) $E(\mathcal{L})$ is irreducible. 
Proof By Lemma 14.3, $X^{\prime \prime}$ has at least two boundary components. Hence $\mathcal{T} \neq \varnothing$, and (1) follows.

If $X^{\prime}$ is prime that by construction $\mathcal{T}=\partial X^{\prime} \backslash T_{1}$; hence $E(\mathcal{L}) \cong X^{\prime}$ and (2) follows. Otherwise, recall the definition of $S$ and $X^{*} \subset X^{\prime}$ from the construction of $\psi$ above. Let $X^{* *}=\operatorname{cl}\left(X^{\prime} \backslash X^{*}\right)$. Since $M$ is hyperbolic the boundary $\partial M$ is the image of $T_{1} \subset X^{*}$; after filling $X^{* *}$ we obtain a ball. This shows that $E(\mathcal{L}) \cong X^{*}$; by construction $X^{*}$ is a factor of the prime decomposition of $X^{\prime}$ and hence is itself prime.

Thus, for every $\alpha \in \mathcal{A}_{3}$, there is $I \subset\{1, \ldots, n\}$ such that the following three conditions are satisfied:

(1) $1 \notin I \neq \varnothing$.

(2) $\left\{T_{i}\right\}_{i \in I}$ (which we will denote by $\mathcal{T}$ ) is a union of components of $\phi^{-1}\left(T_{1}^{\prime} \cup T_{2}^{\prime}\right)$.

(3) The cores of the solid tori attached to $\mathcal{T}$ form a link (which we will denote by $\mathcal{L} \subset M$ ) with an irreducible exterior.

Up to finite ambiguity we fix $I$ as above and consider only $\alpha \in \mathcal{A}_{3}$ that fulfill these conditions for the fixed index set $I$. To avoid overly complicated notation we do not rename $\mathcal{A}_{3}$ or $\hat{\mathcal{A}}$.

We are now ready to state and prove the main proposition of this section:

Proposition 14.6 Let $M$ be a hyperbolic manifold with boundary $\partial M$ a single torus that we will denote by $T, X$ a compact connected orientable manifold with boundary $\partial X$ consisting of tori that we will denote by $\left\{T_{i}\right\}_{i=1}^{n}$, and $\mathcal{A}_{3}$ a set of multislopes on $\partial X$. Fix a nonempty index set $I \subset\{2, \ldots, n\}$; we will denote $\left\{T_{i}\right\}_{i \in I}$ by $\mathcal{T}$. Denote the link formed by the core of the solid tori attached to $\mathcal{T}$ when filling along $\alpha \in \mathcal{A}_{3}$ by $\mathcal{L}_{\alpha} \subset M$ (or simply $\mathcal{L}$ when no confusion may arise). Assume that any $\alpha \in \mathcal{A}_{3}$ fulfills the following conditions:

(1) $\left.\alpha\right|_{T_{1}}=\infty$

(2) $X(\alpha) \cong M$

(3) $E\left(\mathcal{L}_{\alpha}\right)$ is irreducible.

Then for each $i \in I$, there is a bounded set $B_{i}$ of slopes of $T_{i}$ such that for each $\alpha \in \mathcal{A}_{3}$ there is an $i \in I$ with $\left.\alpha\right|_{T_{i}} \in B_{i}$. 
Remark 14.7 By the discussion above (in particular Lemma 14.5), $X, T_{1}, \mathcal{T}$, and the multislopes of $\mathcal{A}$ considered in Case 3 of Theorem 1.1 satisfy the conditions of Proposition 14.6 for some $I$. By Lemma 14.1, proving this proposition will complete the proof of Theorem 1.1.

\section{Proof of Proposition 14.6 We induct on $|T(X)|$.}

Assume that $\boldsymbol{X}$ is Seifert fibered or sol Then no filling of $X$ gives $M$. We assume from now on that $X$ is not Seifert fibered or sol.

Assume that $\boldsymbol{X}$ is hyperbolic Fix $\epsilon>0$ smaller than the length of the shortest geodesic in $M$. By Theorem 9.1 there are only finitely many totally hyperbolic fillings on $X$ yielding $M$. Given $i \in I$, we will denote the set of slopes obtained by restricting totally hyperbolic multislopes of $\mathcal{A}$ to $T_{i}$ by $B_{i}^{1}$, whenever the restriction does not equal $\infty$. Then $B_{i}^{1}$ is finite (and hence bounded). We will denote by $\mathcal{A}^{\prime} \subset \mathcal{A}_{3}$ the set

$$
\mathcal{A}^{\prime}=\left\{\alpha \in \mathcal{A}_{3}|\alpha|_{T_{i}} \notin B_{i}^{1} \text { for any } i \in I\right\} .
$$

From this point on we assume as we may that $\alpha \in \mathcal{A}^{\prime}$. Then $\alpha$ is not totally hyperbolic and hence admits a minimally nonhyperbolic partial filling. For each $i \in I$, let $B_{i}^{2}$ be the set of restrictions $\left\{\left.\alpha_{\min }\right|_{T_{i}}\right\}$, where $\alpha_{\min }$ ranges over all minimally nonhyperbolic fillings for which $\left.\alpha_{\min }\right|_{T_{i}} \neq \infty$. By Proposition 4.2 we have that $X$ admits only finitely many minimally nonhyperbolic fillings and so $B_{i}^{2}$ is finite (and hence bounded). We will denote by $\mathcal{A}^{\prime \prime} \subset \mathcal{A}^{\prime}$ the set

$$
\mathcal{A}^{\prime \prime}=\left\{\alpha \in \mathcal{A}_{3}|\alpha|_{T_{i}} \notin\left(B_{i}^{1} \cup B_{i}^{2}\right) \text { for any } i \in I\right\} .
$$

From this point on we assume as we may that $\alpha \in \mathcal{A}^{\prime \prime}$. By definition, any $\alpha \in \mathcal{A}^{\prime \prime}$ admits a minimally nonhyperbolic partial filling $\alpha_{\min }$ such that $\left.\alpha_{\min }\right|_{T_{i}}=\infty$ for every $i \in I$.

Fix a minimally nonhyperbolic filling $\alpha_{\min }$ for which $\left.\alpha_{\min }\right|_{T_{i}}=\infty$ for every $i \in I$. We will denote $X\left(\alpha_{\min }\right)$ by $X_{1}$ and the restrictions to $X_{1}$ of multislopes in $\mathcal{A}^{\prime \prime}$ that admit a partial filling $\alpha_{\min }$ by $\mathcal{A}_{\alpha_{\min }}$, that is

$$
\mathcal{A}_{\alpha_{\min }}=\left\{\left.\alpha\right|_{\partial X_{1}} \mid \alpha \in \mathcal{A}^{\prime \prime} \text { and } \alpha_{\min } \subset_{\mathrm{pf}} \alpha\right\} .
$$

We claim that the following conditions are satisfied:

(1) $\left|T\left(X_{1}\right)\right|<|T(X)|$ : this holds since $X_{1}$ corresponds to a direct descendant of the root of $T(X)$.

(2) $\mathcal{T} \subset \partial X_{1}$ : this follows from the definition of $\mathcal{A}^{\prime \prime}$, where we required that $\left.\alpha\right|_{T_{i}}=\infty$ (for all $i \in I$ ). 
(3) $\left.\quad \alpha_{1}\right|_{T_{1}}=\infty$ for any $\alpha_{1} \in \mathcal{A}_{\alpha_{\min }}$ : it follows from the definitions that $\left.\alpha_{1}\right|_{T_{1}}=\left.\alpha\right|_{T_{1}}$; hence by the first assumption of the proposition, $\left.\alpha\right|_{T_{1}}=\infty$.

(4) $X_{1}\left(\alpha_{1}\right) \cong M$ for any $\alpha_{1} \in \mathcal{A}_{\alpha_{\min }}$ : by definition, $X_{1}\left(\alpha_{1}\right)=X\left(\alpha_{\min }\right)\left(\alpha_{1}\right)=X(\alpha)$. By the second assumption of the proposition, $X(\alpha) \cong M$.

(5) $E\left(\mathcal{L}_{\alpha_{i}}\right)$ is irreducible for any $\alpha_{1} \in \mathcal{A}_{\alpha_{\text {min }}}$, where $\mathcal{L}_{\alpha_{1}}$ denotes the link formed by the cores of the solid tori attached to $\mathcal{T} \subset \partial X_{1}$ : it is straightforward to see that $E\left(\mathcal{L}_{\alpha_{1}}\right)=E(\mathcal{L})$. By the third assumption of the proposition, $E(\mathcal{L})$ is irreducible.

By (2)-(5), $X_{1}, T_{1}, \mathcal{T}$, and $\mathcal{A}_{\alpha_{\min }}$ fulfill the assumptions of the proposition. By (1) we may apply induction, showing that for every $i \in I$, there is a bounded set of slopes of $T_{i}$, that we will denote by $B_{i, \alpha_{\min }}^{3}$, such that for each $\alpha_{1} \in \mathcal{A}_{\alpha_{\min }}$ there is an $i \in I$ with $\left.\alpha_{1}\right|_{T_{i}} \in B_{i, \alpha_{\min }}^{3}$.

With the notation of the preceding paragraph, every $\alpha \in \mathcal{A}^{\prime \prime}$ admits a minimally nonhyperbolic filling $\alpha_{\min }$ and $\left.\alpha\right|_{T_{i}}=\left.\alpha_{1}\right|_{T_{i}}$ for every $i \in I$. Hence for some $i \in I$, $\left.\alpha\right|_{T_{i}} \in B_{i, \alpha_{\min }}^{3}$. By Proposition $4.2, X$ admits only finitely many minimally nonhyperbolic fillings. Hence the set

$$
B_{i}^{3}=\bigcup_{\alpha_{\min }} B_{i, \alpha_{\min }}^{3}
$$

is bounded. The proposition follows by setting

$$
B_{i}=B_{i}^{1} \cup B_{i}^{2} \cup B_{i}^{3}
$$

We assume from now on that $X$ is not Seifert fibered, sol, or hyperbolic.

Assume that $X$ is reducible Let $X_{1}$ be the factor of the prime decomposition of $X$ that contains $T_{1}$; say $X=X_{1} \# X_{1}^{\prime}$ (we are not assuming that $X_{1}^{\prime}$ is prime). Then any $\alpha \in \mathcal{A}$ induces the multislopes $\alpha_{1}=\left.\alpha\right|_{\partial X_{1}}$ and $\alpha_{1}^{\prime}=\left.\alpha\right|_{\partial X_{1}^{\prime}}$ on $\partial X_{1}$ and $\partial X_{1}^{\prime}$, respectively. Since $X_{1}\left(\alpha_{1}\right) \# X_{1}^{\prime}\left(\alpha_{1}^{\prime}\right)=X(\alpha) \cong M$ and $M$ is hyperbolic, the following conditions hold:

(1) $X_{1}\left(\alpha_{1}\right) \cong M$ since $T_{1} \subset \partial X_{1}$ (with $\left.\left.\alpha_{1}\right|_{T_{1}}=\infty\right)$.

(2) $X_{1}^{\prime}\left(\alpha_{1}^{\prime}\right) \cong S^{3}$.

By construction, the reducing sphere that gives the decomposition $X=X_{1} \# X_{1}^{\prime}$ is disjoint from $\mathcal{L}$. Since $E(\mathcal{L})$ is irreducible, no component of it is contained in $X_{1}^{\prime}\left(\alpha_{1}^{\prime}\right)$; equivalently, $\mathcal{T} \subset \partial X_{1}$. It is easy to see that $X_{1}, T_{1}, \mathcal{T}$, and $\left\{\alpha_{1} \mid \alpha \in \mathcal{A}\right\}$ fulfill the assumptions of the proposition. Since $X_{1}$ corresponds to a direct descendant of the root of $T(X)$, we have $\left|T\left(X_{1}\right)\right|<|T(X)|$. By induction, for each $i \in I$ there is a bounded 


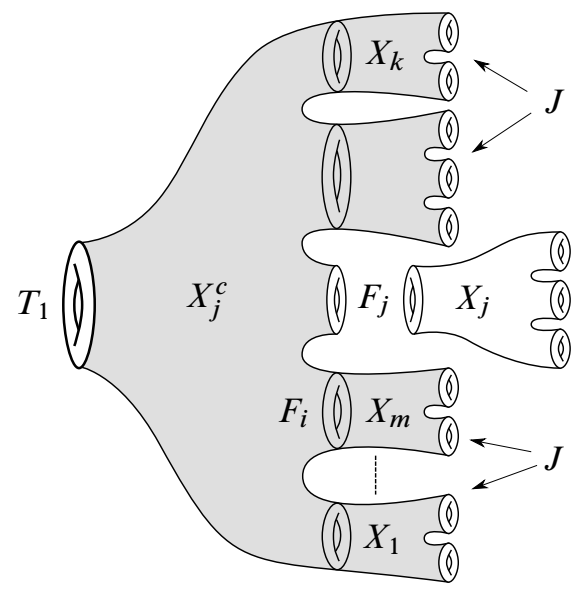

Figure 5: The notation when $X$ is JSJ (the shaded region is $X_{j}^{c}$ )

set of slopes $B_{i}$ of $T_{i}$ such that for each $\alpha \in \mathcal{A}$ there is an $i \in I$ with $\left.\alpha_{1}\right|_{T_{1}} \in B_{i}$. Since $\left.\alpha\right|_{T_{i}}=\left.\alpha_{1}\right|_{T_{i}}$ for all $i \in I$, the proposition follows in this case.

We assume from now on that $X$ is irreducible and not Seifert fibered, sol, or hyperbolic.

Assume that $\boldsymbol{X}$ is JSJ Let $X_{0}$ be the component of the torus decomposition of $X$ that contains $T_{1}$. Denote the components of $\partial X_{0} \backslash T_{1}$ by $\left\{F_{j}\right\}_{j=1}^{k}$. Denote the component of $\operatorname{cl}\left(X \backslash X_{0}\right)$ containing $F_{j}$ by $X_{j}$; see Figure 5. To avoid the situation where $X_{j}=\varnothing$, if $F_{j} \subset \partial X$ we push it slightly into the interior of $X$ so that $X_{j} \cong T^{2} \times[0,1]$ in that case. Since $M$ is hyperbolic it admits no nonseparating tori; thus we assume as we may that $X_{j} \neq X_{j^{\prime}}$ for $j \neq j^{\prime}$, for otherwise no filling of $X$ yields $M$. By renumbering if necessary, we assume as we may that $\partial X_{j}$ contains a component of $\mathcal{T}$ exactly when $j \leq m$, for an appropriately chosen $m$.

For $\alpha \in \mathcal{A}$ we will denote the restriction $\left.\alpha\right|_{\partial X_{j}}$ as $\alpha_{j}$ (by definition $\left.\alpha_{j}\right|_{F_{j}}=\infty$ ). Since $X(\alpha) \cong M$ is hyperbolic, every torus in $X(\alpha)$ is either boundary parallel (Case A below), or bounds a solid torus (Cases B and C below), or bounds a nontrivial knot exterior in a ball (Case D below). Thus for every $1 \leq j \leq k$ exactly one of the following holds:

Case A $X_{j}\left(\alpha_{j}\right) \cong M$ and $\operatorname{cl}\left(X(\alpha) \backslash X_{j}\left(\alpha_{j}\right)\right) \cong T^{2} \times[0,1]$.

Case B $X_{j}\left(\alpha_{j}\right) \cong D^{2} \times S^{1}$ and no component of $\mathcal{L} \cap X_{j}\left(\alpha_{j}\right)$ is a core of $X_{j}\left(\alpha_{j}\right)$.

Case C $X_{j}\left(\alpha_{j}\right) \cong D^{2} \times S^{1}$ and some component of $\mathcal{L} \cap X_{j}\left(\alpha_{j}\right)$, which we will denote by $K_{j}$, is a core of $X_{j}\left(\alpha_{j}\right)$.

Case D $X_{j}\left(\alpha_{j}\right) \cong E\left(K_{j}\right)$ for a nontrivial knot $K_{j} \subset S^{3}$ and $X_{j}\left(\alpha_{j}\right) \subset D_{j}$ for some ball $D_{j} \subset X(\alpha)$. 
We first consider the following:

Case A happens for some $\boldsymbol{j} \leq \boldsymbol{m}$ Fix $j(1 \leq j \leq m)$ and let $\mathcal{A}_{j}^{\prime} \subset \mathcal{A}$ be

$$
\mathcal{A}_{j}^{\prime}=\left\{\alpha \in \mathcal{A} \mid X_{j}\left(\alpha_{j}\right) \cong M\right\} .
$$

Note that for any $\alpha \in \mathcal{A}$, if $F_{j}$ is as in Case A above then $\alpha \in \mathcal{A}_{j}^{\prime}$. We will denote the set of restrictions $\left\{\alpha_{j} \mid \alpha \in \mathcal{A}_{j}^{\prime}\right\}$ by $\mathcal{A}_{j}$. Let $\mathcal{T}_{j}=\mathcal{T} \cap X_{j}$ and $\mathcal{L}_{j}=\mathcal{L} \cap X_{j}\left(\alpha_{j}\right)$; equivalently, $\mathcal{L}_{j}$ is the link formed by the cores of the solid tori attached to $\mathcal{T}_{j}$. Since $j \leq m$, we have $\mathcal{T}_{j} \neq \varnothing$. If $\mathcal{L}_{j}$ were reducible for some $\alpha_{j} \in \mathcal{A}_{j}$ then any reducing sphere for $X_{j}\left(\alpha_{j}\right) \backslash$ int $N\left(\mathcal{L}_{j}\right)$ would be a reducing sphere for $\mathcal{L}$; this contradicts the assumptions of the proposition.

Hence $X_{j}, F_{j}, \mathcal{T}_{j}$ and $\mathcal{A}_{j}$ fulfill the assumptions of the proposition. Since $X_{j}$ is a direct descendant of the root of $T(X)$, we have that $\left|T\left(X_{j}\right)\right|<|T(X)|$. By induction on each component $T$ of $\mathcal{T}_{j}$, there is a bounded set of slopes, that we will denote by $B_{T}$, such that for each $\alpha_{j} \in \mathcal{A}_{j}$ there is a component $T$ of $\mathcal{T}_{j}$ with $\left.\alpha_{j}\right|_{T} \in B_{T}$. It follows that for every $\alpha \in \mathcal{A}_{j}^{\prime}$ we have $\left.\alpha\right|_{T} \in B_{T}$ for some $T$; the proposition follows in this case.

We may consider from now on only multislopes from $\mathcal{A} \backslash\left(\bigcup_{j \leq m} \mathcal{A}_{j}^{\prime}\right)$ (and in particular, we assume as we may that Case A above does not happen for $j \leq m$ ). To avoid overly complicated notation we do not rename $\mathcal{A}$.

Next we consider the following:

Case A happens for some $\boldsymbol{j} \geq \boldsymbol{m}+\mathbf{1}$ Fix $j(m+1 \leq j \leq k)$. We will denote $\operatorname{cl}\left(X \backslash X_{j}\right)$ by $X_{j}^{c}$ and the restriction $\left.\alpha\right|_{\partial X_{j}^{c}}$ by $\alpha_{j}^{c}$; note that $\left.\alpha_{j}^{c}\right|_{F_{j}}$ and $\left.\alpha_{j}^{c}\right|_{T_{1}}$ are $\infty$. Since $j \geq m+1, \mathcal{T} \subset \partial X_{j}^{c}$. Let $\mathcal{A}_{j}^{\prime} \subset \mathcal{A}$ be the set

$$
\mathcal{A}_{j}^{\prime}=\left\{\alpha \in \mathcal{A} \mid X_{j}^{c}\left(\alpha_{j}^{c}\right) \cong T^{2} \times[0,1]\right\} .
$$

Note that for any $\alpha \in \mathcal{A}, F_{j}$ is as in Case A above if and only if $\alpha \in \mathcal{A}_{j}^{\prime}$. We will denote the set of restrictions $\left\{\alpha_{j}^{c} \mid \alpha \in \mathcal{A}_{j}^{\prime}\right\}$ by $\mathcal{A}_{j}^{c}$.

Thus we have a manifold $X_{j}^{c}$ such that $\mathcal{T} \subset \partial X_{j}^{c}$ and multislopes $\mathcal{A}_{j}^{c}$ such that $X_{j}^{c}\left(\alpha_{j}^{c}\right) \cong T^{2} \times[0,1]$ and $\partial X_{j}^{c}\left(\alpha_{j}^{c}\right)=T_{1} \cup F_{j}$ for every $\alpha_{j}^{c} \in \mathcal{A}_{j}^{c}$. Up to finite ambiguity we fix $J \subset\{1, \ldots, k\}$, and denote by $\mathcal{A}_{J}^{c} \subset \mathcal{A}_{j}^{c}$ the multislopes for which $X_{j^{\prime}}\left(\alpha_{j^{\prime}}\right) \cong E\left(K_{j^{\prime}}\right) \subset D_{j^{\prime}}$ for a nontrivial knot $K_{j^{\prime}} \subset S^{3}$ and a ball $D_{j^{\prime}} \subset X(\alpha)$ if and only if $j^{\prime} \in J$. By Lemma 2.2 we may assume that the balls $\left\{D_{j^{\prime}}\right\}_{j^{\prime} \in J}$ are disjointly embedded.

Let $Y^{\prime}$ be the closure of $X_{j}^{c} \backslash\left(\bigcup_{j^{\prime} \in J} E\left(K_{j^{\prime}}\right)\right.$ ) (see Figure 6; in that figure $\left\{F_{j^{\prime}}\right\}_{j^{\prime} \in J}$ are the two tori on the top right). By restriction, any $\alpha_{j}^{c} \in \mathcal{A}_{J}^{c}$ induces a slope on 


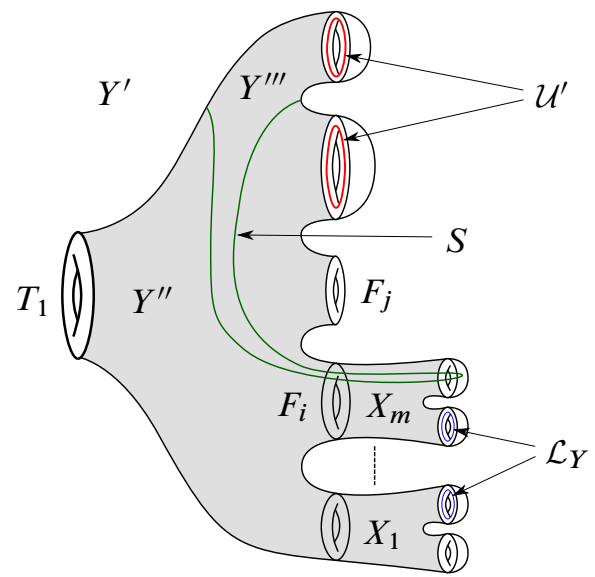

Figure 6: $Y^{\prime}\left(\alpha_{Y^{\prime}}\right)$

each component of $\partial Y^{\prime} \backslash\left(T_{1} \cup F_{j} \cup\left(\bigcup_{j^{\prime} \in J} F_{j^{\prime}}\right)\right)$. For every $j^{\prime} \in J$, we pick a slope on $F_{j^{\prime}}$ that intersects the meridian of $E\left(K_{j^{\prime}}\right)$ exactly once. (There are infinitely many ways to do this; we will exploit this flexibility soon when appealing to Lemma 2.4.) Denote the multislope obtained by $\alpha_{Y^{\prime}}$, and note that $Y^{\prime}\left(\alpha_{Y^{\prime}}\right) \cong T^{2} \times[0,1]$ and $\partial Y^{\prime}\left(\alpha_{Y^{\prime}}\right)=T_{1} \cup F_{j}$. We will denote $\mathcal{T} \cap \partial Y^{\prime}$ by $\mathcal{T}_{Y}$, the link formed by the cores of the solid tori attached to $\mathcal{T}_{Y}$ by $\mathcal{L}_{Y}$, and the link formed by the cores of the solid tori attached to $\bigcup_{j^{\prime} \in J} F_{j^{\prime}}$ by $\mathcal{U}^{\prime}$ (in Figure $6, \mathcal{U}^{\prime}$ is red). Since the components of $\mathcal{U}^{\prime}$ are unknots contained in the disjointly embedded balls $D_{j^{\prime}}, \mathcal{U}^{\prime}$ is an unlink. Finally assume, for a contradiction, that $\mathcal{T}_{Y}=\varnothing$. Then $\mathcal{L} \subset \bigcup_{j^{\prime} \in J} D_{j^{\prime}}$; this contradicts the assumption that $E(\mathcal{L})$ is irreducible, showing that $\mathcal{T}_{Y} \neq \varnothing$.

If $Y^{\prime}\left(\alpha_{Y^{\prime}}\right) \backslash \operatorname{int} N\left(\mathcal{L}_{Y} \cup \mathcal{U}^{\prime}\right)$ is irreducible we denote $Y^{\prime}$ by $Y, \mathcal{U}^{\prime}$ by $\mathcal{U}$, and $\alpha_{Y^{\prime}}$ by $\alpha_{Y}$. Otherwise, let $S$ be a reducing sphere that realizes the decomposition of $Y^{\prime}\left(\alpha_{Y^{\prime}}\right) \backslash$ int $N\left(\mathcal{L}_{Y} \cup \mathcal{U}^{\prime}\right)$ as $Y^{\prime \prime} \# Y^{\prime \prime \prime}$, where $Y^{\prime \prime}$ is irreducible and $T_{1} \subset Y^{\prime \prime}$ (in Figure $6, S$ is green). Note that $Y^{\prime}\left(\alpha_{Y^{\prime}}\right) \backslash$ int $N\left(\mathcal{L}_{Y} \cup \mathcal{U}^{\prime}\right)$ is obtained from $Y^{\prime}$ by filling $\partial Y^{\prime} \backslash\left(T_{1} \cup F_{j} \cup \mathcal{T}_{Y} \cup\left(\bigcup_{j^{\prime} \in J} F_{j^{\prime}}\right)\right)$ (in Figure 6 the components that are not filled are $T_{1}, F_{j}, \mathcal{U}^{\prime}$ and $\left.\mathcal{L}_{Y}\right)$. Since $\mathcal{T} \subset \mathcal{T}_{Y} \cup\left(\bigcup_{j^{\prime} \in J} \partial X_{j^{\prime}}\right)$, we have $\mathcal{L} \cap S=\varnothing$. We consider $S$ as a sphere in

$$
Y^{\prime}\left(\alpha_{Y^{\prime}}\right) \cup_{F_{j}} X_{j}\left(\alpha_{j}\right) \cong T^{2} \times[0,1] \cup_{F_{j}} M \cong M .
$$

Since $M$ is hyperbolic, $S$ bounds a ball in $Y^{\prime}\left(\alpha_{Y^{\prime}}\right) \cup_{F_{j}} X_{j}\left(\alpha_{j}\right)$ which we will denote by $D$. Since $T_{1} \subset Y^{\prime \prime}$, we have $D=Y^{\prime \prime \prime}\left(\left.\alpha_{Y^{\prime}}\right|_{Y^{\prime \prime \prime}}\right)$. Clearly, $\mathcal{T}_{Y} \cap D=\varnothing$, for otherwise $S$ would be a reducing sphere for $E(\mathcal{L})$. We will denote by $Y$ the manifold obtained from $Y^{\prime}$ by filling $\left(\bigcup_{j^{\prime} \in J} F_{j^{\prime}}\right) \cap D$ along the multislope induced by $\alpha_{Y^{\prime}}$. We will denote the multislope $\left.\alpha_{Y^{\prime}}\right|_{\partial Y}$ by $\alpha_{Y}$ and $\mathcal{U}^{\prime} \backslash\left(\mathcal{U}^{\prime} \cap D\right)$ by $\mathcal{U}$. By construction 
$\mathcal{T}_{Y} \subset \partial Y$, and therefore we may consider $\mathcal{L}_{Y}$ as the link in $Y\left(\alpha_{Y}\right)$ formed by the cores of the solid tori attached to $\mathcal{T}_{Y}$. Then the following two conditions hold:

(1) $\mathcal{U}$ is an unlink.

(2) $Y\left(\left.\alpha\right|_{Y}\right) \backslash$ int $N\left(\mathcal{L}_{Y} \cup \mathcal{U}\right) \cong Y^{\prime \prime}$ and hence is irreducible.

We choose the slopes of $\mathcal{U}$, as we know we may by Lemma 2.4 , so that $E\left(\mathcal{L}_{Y}\right)$ is irreducible.

Lemma 14.8 For every $\alpha_{Y} \in \mathcal{A}_{Y}$, there is a slope $\alpha^{\prime}$ of $F_{j}$ such that $Y\left(\alpha_{Y}\right)\left(\alpha^{\prime}\right)$ satisfies the following conditions:

(1) $Y\left(\alpha_{Y}\right)\left(\alpha^{\prime}\right) \cong D^{2} \times S^{1}$.

(2) $\mathcal{L}_{Y} \subset Y\left(\alpha_{Y}\right)\left(\alpha^{\prime}\right)$ is irreducible.

(3) No component of $\mathcal{L}_{Y}$ is a core of $Y\left(\alpha_{Y}\right)\left(\alpha^{\prime}\right)$.

\section{Proof of Lemma 14.8 Fix $\alpha_{Y} \in \mathcal{A}_{Y}$.}

By construction, $Y\left(\alpha_{Y}\right) \cong T^{2} \times[0,1]$; hence for any slope $\alpha^{\prime}$ we have $Y\left(\alpha_{Y}\right)\left(\alpha^{\prime}\right) \cong$ $D^{2} \times S^{1}$. Thus (1) is satisfied by any slope $\alpha^{\prime}$ of $F_{j}$.

Since the exterior of $\mathcal{L}_{Y}$ as a link in $Y\left(\alpha_{Y}\right)$ is irreducible, by Hatcher [7, Corollary], there is a finite set of slopes of $F_{j}$, which we will denote by $B_{f}$, such that for any slope $\alpha^{\prime} \notin B_{f}$ the exterior of $\mathcal{L}_{Y}$ as a link in $Y\left(\alpha_{Y}\right)\left(\alpha^{\prime}\right)$ is irreducible.

For (3) we fix a component $K$ of $\mathcal{L}_{Y}$. Let $[K]$ denote the homology class represented by $K$ in $H_{1}\left(Y\left(\alpha_{Y}\right) ; \mathbb{Z}\right)$ ([K] is only defined up to sign). We consider two possibilities:

- $[K]$ is not primitive Then by Lemma $2.6, K$ is not a core of $Y\left(\alpha_{Y}\right)\left(\alpha^{\prime}\right)$ for any slope $\alpha^{\prime}$; we set $B_{K}=\varnothing$.

- $[K]$ is primitive By Lemma 2.6, if $K$ is a core of $Y\left(\alpha_{Y}\right)\left(\alpha^{\prime}\right)$ then $[K]$ and [ $\left.\alpha^{\prime}\right]$ generate $H_{1}\left(Y\left(\alpha_{Y}\right)\right)$. We will denote by $B_{K}$ the set of slopes of $F_{j}$ that correspond to homology classes fulfilling this condition. It is easy to see that $B_{K}$ has diameter 2 in the Farey graph of the slopes of $F_{j}$.

Since $B_{f} \cup\left(\cup_{K} B_{K}\right)$ is a finite union of bounded sets it is itself bounded; hence its complement is not empty. Lemma 14.8 follows by picking $\alpha^{\prime} \notin B_{f} \cup\left(\cup_{K} B_{K}\right)$.

For each $\alpha_{Y} \in \mathcal{A}_{Y}$ we pick a slope $\alpha^{\prime}$ of $F_{j}$ satisfying the conditions of Lemma 14.8. By Proposition 7.2, on every component $T$ of $\mathcal{T}_{Y}$, there exists a bounded set $B_{T}$ such for that every $\alpha_{Y} \in \mathcal{A}_{Y}$ there is a component $T$ of $\mathcal{T}_{Y}$ with $\left.\alpha_{Y}\right|_{T} \in B_{T}$. Given any 
$\alpha \in \mathcal{A}$, we construct $\alpha_{j}^{c} \in \mathcal{A}_{J}^{c}$ and $\alpha_{Y}$ as above. The proposition follows in this case since $\left.\alpha\right|_{T}=\left.\alpha_{j}^{c}\right|_{T}=\left.\alpha_{Y}\right|_{T} \in B_{T}$.

We may consider from now on only multislopes from $\mathcal{A} \backslash\left(\bigcup_{j \geq m+1} \mathcal{A}_{j}^{\prime}\right)$. Thus from now on we will only consider multislopes for which Case A does not happen for any $j$. To avoid overly complicated notation we do not rename $\mathcal{A}$.

Next we consider the following:

Case B happens for some $\boldsymbol{j} \leq \boldsymbol{m}$ Fix $1 \leq j \leq m$. We will denote by $\mathcal{A}_{j}^{\prime} \subset \mathcal{A}$ the multislopes $\alpha \in \mathcal{A}$ for which $X_{j}\left(\alpha_{j}\right) \cong D^{2} \times S^{1}$ and no component of $\mathcal{L} \cap X_{j}\left(\alpha_{j}\right)$ is a core. Note that Case B occurs if and only if $\alpha \in \mathcal{A}_{j}^{\prime}$.

We will denote the set of restrictions $\left\{\alpha_{j} \mid \alpha \in \mathcal{A}_{j}^{\prime}\right\}$ by $\mathcal{A}_{j}$ and $\mathcal{T} \cap X_{j}$ by $\mathcal{T}_{j}$. Since $j \leq m$, we have $\mathcal{T}_{j} \neq \varnothing$. Given $\alpha_{j} \in \mathcal{A}_{j}$, we will denote the link formed by the cores of the solid tori attached to $\mathcal{T}_{j}$ by $\mathcal{L}_{j}$. It is easy to see that if $X_{j}\left(\alpha_{j}\right) \backslash$ int $N\left(\mathcal{L}_{j}\right)$ were reducible then $E(\mathcal{L})$ would be reducible, contradicting the third assumption of the proposition. By the assumption of Case $\mathrm{B}$, no component of $\mathcal{L}_{j}$ is a core of the solid torus $X_{j}\left(\alpha_{j}\right)$. Therefore by Proposition 7.2, for each component $T$ of $\mathcal{T}_{j}$, there is a bounded set of slopes of $T$, which we will denote by $B_{T}$, such that for each $\alpha_{j} \in \mathcal{A}_{j}$ there is a component $T$ of $\mathcal{T}_{j}$ with $\left.\alpha_{j}\right|_{T} \in B_{T}$. The proposition follows in this case since $\left.\alpha\right|_{T}=\left.\alpha_{j}\right|_{T}$ for any $\alpha \in \mathcal{A}_{j}^{\prime}$.

We may consider from now on only multislopes from $\mathcal{A} \backslash\left(\bigcup_{j \leq m} \mathcal{A}_{j}^{\prime}\right)$. To avoid overly complicated notation we do not rename $\mathcal{A}$.

We have reduced the proof to the following:

Case A never happens and Case B never happens for $\boldsymbol{j} \leq \boldsymbol{m}$ Consider $J_{1}, J_{2} \subset$ $\{1, \ldots, k\}$ fulfilling the following conditions:

(1) $\varnothing \neq J_{1} \subset\{1, \ldots, m\}$.

(2) $J_{1} \cap J_{2}=\varnothing$.

(3) $\{1, \ldots, m\} \subset J_{1} \cup J_{2}$.

Let $\mathcal{A}_{J_{1}, J_{2}} \subset \mathcal{A}$ be the multislopes $\alpha$ fulfilling the following conditions:

(1) $X_{j}\left(\alpha_{j}\right) \cong D^{2} \times S^{1}$ for every $j \in J_{1}$.

(2) $X_{j}\left(\alpha_{j}\right) \cong E\left(K_{j}\right)$ for any $1 \leq j \leq k$ (for a nontrivial knot $K_{j} \subset S^{3}$ ) if and only if $j \in J_{2}$.

Using the fact that Case A does not happen (that is, every $F_{j}$ bounds a solid torus or a knot exterior contained in a ball) and irreducibility of $\mathcal{L}$ and Lemma 2.2 (which 
together imply that $\left.J_{1} \neq \varnothing\right)$, it is easy to see that for any $\alpha \in \mathcal{A}$ there is a choice of $J_{1}, J_{2}$ as above for which $\alpha \in \mathcal{A}_{J_{1}, J_{2}}$; thus

$$
\mathcal{A}=\bigcup_{J_{1}, J_{2}} \mathcal{A}_{J_{1}, J_{2}} .
$$

Up to finite ambiguity we fix $J_{1}, J_{2} \subset\{1, \ldots, k\}$ fulfilling the conditions above and consider only multislopes from $\mathcal{A}_{J_{1}, J_{2}}$.

Fix a multislope $\alpha \in \mathcal{A}_{J_{1}, J_{2}}$.

By Lemma 2.2 we may fix disjointly embedded balls $\left\{D_{j}\right\}_{j \in J_{2}}$ such that $E\left(K_{j}\right) \subset D_{j}$. Since Case B does not happen for $j \leq m$, for every $j \in J_{1}$, at least one component of $\mathcal{L}$ is a core of $X_{j}\left(\alpha_{j}\right)$; we choose one and denote it by $K_{j}$. We will denote $\bigcup_{j \in J_{1}} K_{j}$ by $\mathcal{L}_{1}$ and $\bigcup_{j \in J_{1}} T_{j}$ by $\mathcal{T}_{1}$.

The multislope $\alpha$ induces a multislope on $\partial X_{0}$ as follows: for $j \notin J_{2}$, the slope induced on $F_{j}$ is the meridian of solid torus $X_{j}\left(\alpha_{j}\right)$. For $j \in J_{2}$, the slope induced on $F_{j}$ is any slope that intersects the meridian of $E\left(K_{j}\right)$ once (we will exploit this flexibility soon, when appealing to Lemma 2.4). We will denote the multislope induced by $\alpha$ on $\partial X_{0}$ by $\alpha_{0}$. By construction $X_{0}\left(\alpha_{0}\right) \cong M$. For $j \in J_{1}$, we will denote the core of the solid torus attached to $F_{j}$ by $K_{j}$ and the link formed by the cores of the solid tori attached to $\mathcal{T}_{1}$ by $\mathcal{L}_{1}$; no confusion should arise from this notation, as $K_{j}$ and $\mathcal{L}_{1}$ are isotopic to the knots and link denoted that way previously. We will denote the link formed by the solid tori attached to $\bigcup_{j \in J_{2}} F_{j}$ by $\mathcal{U}$. By construction, the components of $\mathcal{U}$ are unknots embedded in the disjoint balls $D_{j}$, and hence $\mathcal{U}$ is an unlink.

In order to apply Lemma 2.4 we need to know that $\mathcal{L}_{1}$ is irreducible in the complement of $\mathcal{U}$; this is not quite the case, but we can obtain this by considering only some of the components of $\mathcal{U}$. To that end we prove:

Lemma 14.9 Suppose $S$ is a reducing sphere for $\mathcal{L}_{1}$ in the complement of $\mathcal{U}$. Then $S$ bounds a ball $D \subset X_{0}\left(\alpha_{0}\right)$ such that $D \cap \mathcal{L}_{1}=\varnothing$ and $D$ contains at least one component of $\mathcal{U}$.

Proof of Lemma 14.9 Let $S$ be a reducing sphere for $\mathcal{L}_{1}$ in $X_{0}\left(\alpha_{0}\right) \backslash N(\mathcal{U})$; equivalently, $S$ is a reducing sphere for $X_{0}\left(\alpha_{0}\right) \backslash N\left(\mathcal{U} \cup \mathcal{L}_{1}\right)$. Fix $1 \leq j \leq m$; note that either $j \in J_{1}$ or $j \in J_{2}$. If $j \in J_{2}$, then by construction $S$ is disjoint from $F_{j}$. If $j \in J_{1}$, then $S \cap K_{j}=\varnothing$ (since $\left.K_{j} \subset \mathcal{L}_{1}\right)$. Since $K_{j}$ is a core of the solid torus attached to $F_{j}$, we may isotope $S$ out of this solid torus without intersecting $K_{j}$. After performing this isotopy (if necessary) for each $1 \leq j \leq m$, the reducing sphere $S$ is disjoint from $F_{j}$ for every $1 \leq j \leq m$. Since $X_{0}\left(\alpha_{0}\right) \cong X(\alpha)$, we may consider $S$ as a sphere in $X(\alpha)$, 
where we see that $\mathcal{L} \cap S=\varnothing$. Hyperbolicity of $X(\alpha)$ and irreducibility of $E(\mathcal{L})$ imply that $S$ bounds a ball $D \subset X(\alpha)$ such that $\mathcal{L} \cap D=\varnothing$. It follows that $X_{j}\left(\alpha_{j}\right) \cap D=\varnothing$ for $1 \leq j \leq m$; therefore $\mathcal{T}_{1} \cap D=\varnothing$. Hence $S$ bounds a ball in $X_{0}\left(\alpha_{0}\right)$ that is disjoint from $\mathcal{T}_{1}$ and hence from $\mathcal{L}_{1}$. On the other hand, $S$ does not bound a ball in $X_{0}\left(\alpha_{0}\right) \backslash N(\mathcal{U})$; hence $D$ must contain at least one component of $\mathcal{U}$.

This completes the proof of Lemma 14.9.

Let $D$ be as in Lemma 14.9. We remove the components of $\mathcal{U} \cap D$ from $\mathcal{U}$; to avoid overly complicated notation we do not rename $\mathcal{U}$. We repeat this process as long as we can; it terminates since the number of components of $\mathcal{U}$ is reduced. When it does, $\mathcal{L}_{1}$ is irreducible in the complement of the unlink $\mathcal{U}$. By Lemma 2.4 we may change the slopes $\left.\alpha_{0}\right|_{\mathcal{U}}$ so that the exterior of $\mathcal{L}_{1}$ is irreducible. To avoid overly complicated notation we do not rename $\alpha_{0}$

We will denote by $\mathcal{A}_{0}$ the multislopes induced on $\partial X_{0}$ by multislopes of $\mathcal{A}_{J_{1}, J_{2}}$ via the procedure described above. We see that $X_{0}, T_{1}, \mathcal{T}_{1}$, and $\mathcal{A}_{0}$ fulfill the assumptions of the proposition. By Lemma 5.6, we have $\left|T\left(X_{0}\right)\right|<|T(X)|$. By induction, for each component $F_{j}$ of $\mathcal{T}_{1}$, there is a bounded set of slopes of $F_{j}$ which we will denote by $B_{F_{j}}$, such that for each $\alpha_{0} \in \mathcal{A}_{0}$ there is some component $F_{j}$ of $\mathcal{T}_{1}$ with $\left.\alpha_{0}\right|_{F_{j}} \in B_{F_{j}}$.

Given $j \in J_{1}$, let $S \neq F_{j}$ be a component of $\partial X_{j}$. Let $\beta$ be a multislope of $\partial X_{j}$ such that $\left.\beta\right|_{F_{j}}$ and $\left.\beta\right|_{S}$ are both $\infty$ and $X_{j}(\beta) \cong T^{2} \times[0,1]$. We will denote by $B_{\beta}$ the projection of $B_{F_{j}}$ to the slopes of $S$ induced by the product structure on $X_{j}(\beta)$. By the $T^{2} \times[0,1]$ cosmetic surgery theorem (Theorem 6.1), the set $\cup_{\beta} B_{\beta}$ is bounded (where the union is taken over all possible multislopes $\beta$ as above; if there is no such multislope then $\left.\bigcup_{\beta} B_{\beta}=\varnothing\right)$. We will denote $\bigcup_{\beta} B_{\beta}$ by $B_{S}$.

Given $\alpha \in \mathcal{A}_{J_{1}, J_{2}}$, let $\alpha_{0}$ be the induced multislope on $X_{0}$ as above. Let $F_{j}$ be the component for which $\left.\alpha_{0}\right|_{F_{j}} \in B_{F_{j}}$. Recall that $K_{j}$ is a core of a solid torus attached to $X_{j}$ which is also a core of $X_{j}\left(\alpha_{j}\right)$. Let $S$ be the component of $\partial X_{j}$ that corresponds to $K_{j}$. By definition of $\left.\alpha_{0}\right|_{F_{j}}$, it is the projection of $\left.\alpha\right|_{S}$ under the natural projection given by the product structure on $X_{j}\left(\alpha_{j}\right) \backslash N\left(K_{j}\right)$. Thus $\left.\alpha\right|_{S} \in B_{S}$. This shows that for every $\alpha \in \mathcal{A}_{J_{1}, J_{2}}$, there is a boundary component $S$ such that $\left.\alpha\right|_{S} \in B_{S}$. Since $B_{S}$ is bounded, this completes the proof of Proposition 14.6.

\section{References}

[1] J Berge, The knots in $D^{2} \times S^{1}$ which have nontrivial Dehn surgeries that yield $D^{2} \times S^{1}$, Topology Appl. 38 (1991) 1-19 MR1093862 
[2] S A Bleiler, C D Hodgson, J R Weeks, Cosmetic surgery on knots, from "Proceedings of the Kirbyfest" (J Hass, M Scharlemann, editors), Geom. Topol. Monogr. 2, Geom. Topol. Publ., Coventry, UK (1999) 23-34 MR1734400

[3] C Cao, G R Meyerhoff, The orientable cusped hyperbolic 3-manifolds of minimum volume, Invent. Math. 146 (2001) 451-478 MR1869847

[4] D Gabai, Surgery on knots in solid tori, Topology 28 (1989) 1-6 MR991095

[5] M Gromov, Volume and bounded cohomology, Inst. Hautes Études Sci. Publ. Math. 56 (1982) 5-99 MR686042

[6] G H Hardy, E M Wright, An introduction to the theory of numbers, 6th edition, Oxford Univ. Press (2008) MR2445243

[7] A E Hatcher, On the boundary curves of incompressible surfaces, Pacific J. Math. 99 (1982) 373-377 MR658066

[8] H M Hilden, Every closed orientable 3-manifold is a 3-fold branched covering space of $S^{3}$, Bull. Amer. Math. Soc. 80 (1974) 1243-1244 MR0350719

[9] W Jaco, Lectures on three-manifold topology, CBMS Regional Conference Series in Math. 43, Amer. Math. Soc., Providence, RI (1980) MR565450

[10] W H Jaco, P B Shalen, Seifert fibered spaces in 3-manifolds, Mem. Amer. Math. Soc. 220, Amer. Math. Soc., Providence, RI (1979) MR539411

[11] K Johannson, Homotopy equivalences of 3-manifolds with boundaries, Lecture Notes in Math. 761, Springer (1979) MR551744

[12] A Kawauchi, Imitation of (3, 1)-dimensional manifold pairs, Sūgaku 40 (1988) 193204 MR974760 Translated in Sugaku Expositions 2 (1989) 141-156

[13] A Kawauchi, Almost identical imitations of (3, 1)-dimensional manifold pairs, Osaka J. Math. 26 (1989) 743-758 MR1040422

[14] T Kobayashi, Y Rieck, A linear bound on the tetrahedral number of manifolds of bounded volume (after Jorgensen and Thurston), from "Topology and geometry in dimension three" (W Li, L Bartolini, J Johnson, F Luo, R Myers, J H Rubinstein, editors), Contemp. Math. 560, Amer. Math. Soc., Providence, RI (2011) 27-42 MR2866921

[15] JM Montesinos, A representation of closed orientable 3-manifolds as 3-fold branched coverings of $S^{3}$, Bull. Amer. Math. Soc. 80 (1974) 845-846 MR0358784

[16] R Myers, Simple knots in compact, orientable 3-manifolds, Trans. Amer. Math. Soc. 273 (1982) 75-91 MR664030

[17] J Remigio-Juárez, The link volume of 3-manifolds is not multiplicative under coverings, Proc. Amer. Math. Soc. 144 (2016) 4075-4079 MR3513562

[18] J Remigio-Juárez, Y Rieck, The link volumes of some prism manifolds, Algebr. Geom. Topol. 12 (2012) 1649-1665 MR2966698 
[19] Y Rieck, Y Yamashita, The link volume of 3-manifolds, Algebr. Geom. Topol. 13 (2013) 927-958 MR3044597

[20] H Seifert, Topologie Dreidimensionaler Gefaserter Räume, Acta Math. 60 (1933) 147-238 MR1555366

[21] C Series, The modular surface and continued fractions, J. London Math. Soc. 31 (1985) 69-80 MR810563

[22] T Soma, The Gromov invariant of links, Invent. Math. 64 (1981) 445-454 MR632984

[23] M Teragaito, Links with surgery yielding the 3-sphere, J. Knot Theory Ramifications 11 (2002) 105-108 MR1885750

[24] W P Thurston, The geometry and topology of three-manifolds, lecture notes, Princeton University (1979) Available at http://msri.org/publications/books/gt3m

Department of Mathematics, University of Arkansas

Fayetteville, AR 72701, United States

Department of Information and Computer Sciences, Nara Women's University Kitauoya Nishimachi, Nara 630-0586, Japan

yoav@uark.edu, yamasita@ics.nara-wu.ac.jp

Received: 1 October $2015 \quad$ Revised: 24 February 2016 
